\title{
Visible-Light Photocatalysis of Eosin Y: HAT and Complementing MS-CPET Strategy to Trifluoromethylation of $\beta$-Ketodithioesters with Langlois' Reagent
}

Sonam Soni, Pragya Pali, Monish Arbaz Ansari, and Maya Shankar Singh*

Department of Chemistry, Institute of Science, Banaras Hindu University, Varanasi 221005, India

\section{Supporting Information}

\section{Table of Content}

$\begin{array}{ll}\text { Reaction Equipment (Figure S1) S2 } & \text { S2 }\end{array}$

Some Biologically Active Compounds Containing $-\mathrm{SCF}_{3}$ Group (Figure S2) S3

$\begin{array}{ll}\text { Unsuccessful Substrates (Table S1) } & \text { S3 }\end{array}$

$\begin{array}{ll}\text { HRMS Spectra (Figures S3-S8) } & \text { S4-S6 }\end{array}$

NMR Spectra $\left({ }^{1} \mathrm{H},{ }^{13} \mathrm{C}\left\{{ }^{1} \mathrm{H}\right\}\right.$ and $\left.{ }^{19} \mathrm{~F}\right)$ of Compounds (2a-2bd) S7-S51

Cyclic voltammogram of representative compounds 1 in DMF (Figure S9) S52-S53

Cyclic voltammogram of sodium trilflinate in DMF (Figure S10) S53

Cyclic voltammogram of S,S'-bis(dithioacetals) in DMF (Figure S11) S53

$\begin{array}{ll}\text { ORTEP Diagram of Compound 2s } & \text { S54 }\end{array}$

$\begin{array}{ll}\text { Crystallization details } & \text { S55 }\end{array}$

Thermal Ellipsoid Plot of 2s (Figure S12) S55 




Figure S1. Photoreactor 
<smiles>CCNC(Cc1cccc(S(F)(F)F)c1)CN(C)C</smiles>

Tiflorex (Anorectic)<smiles>Cc1cc(C2C(=O)NC(=O)C(C)C2=O)ccc1Oc1ccc(C(F)(F)F)cc1</smiles>

Toltrazuril, Baycox ${ }^{\circledR}$ Bayer (Coccidiostat)

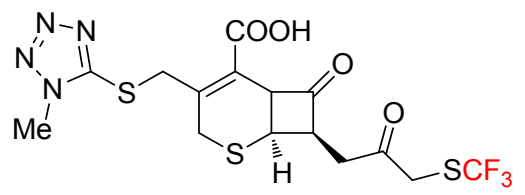

Cefazaflur (Anti-bacterial)

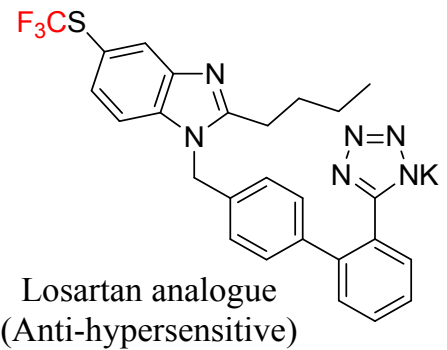

(Anti-hypersensitive)

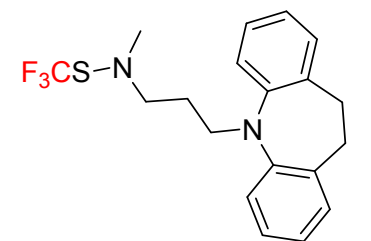

Imipramine analogue, Tof ranil (Antidepressant)<smiles>CC(C)(COc1cc(C#N)ccc1C(F)(F)F)NC(=O)c1ccc(C(F)(F)F)cc1</smiles>

Monepantel, Zolvix ${ }^{\circledR}$ Novartis Sulfur analogue of Riluzole ${ }^{\circledR}$ Sanofi (Anthelmintic drug)<smiles>Nc1nc2ccc(S(F)(F)(F)(F)F)cc2s1</smiles>
(treats amyotrophic lateral sclerosis)

Epiandrosterone analogue (Androgenic steroid harmone)

Figure S2. Some Biologically Active Compounds Containing S-CF 3 Group

Table S1. Unsuccessful Substrates<smiles>C=CSC(=S)CC(=O)c1ccc(Cl)cc1</smiles>

$1 \mathrm{bh}$

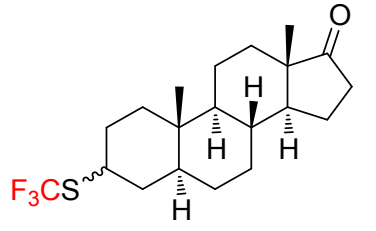

\author{
(1)
}




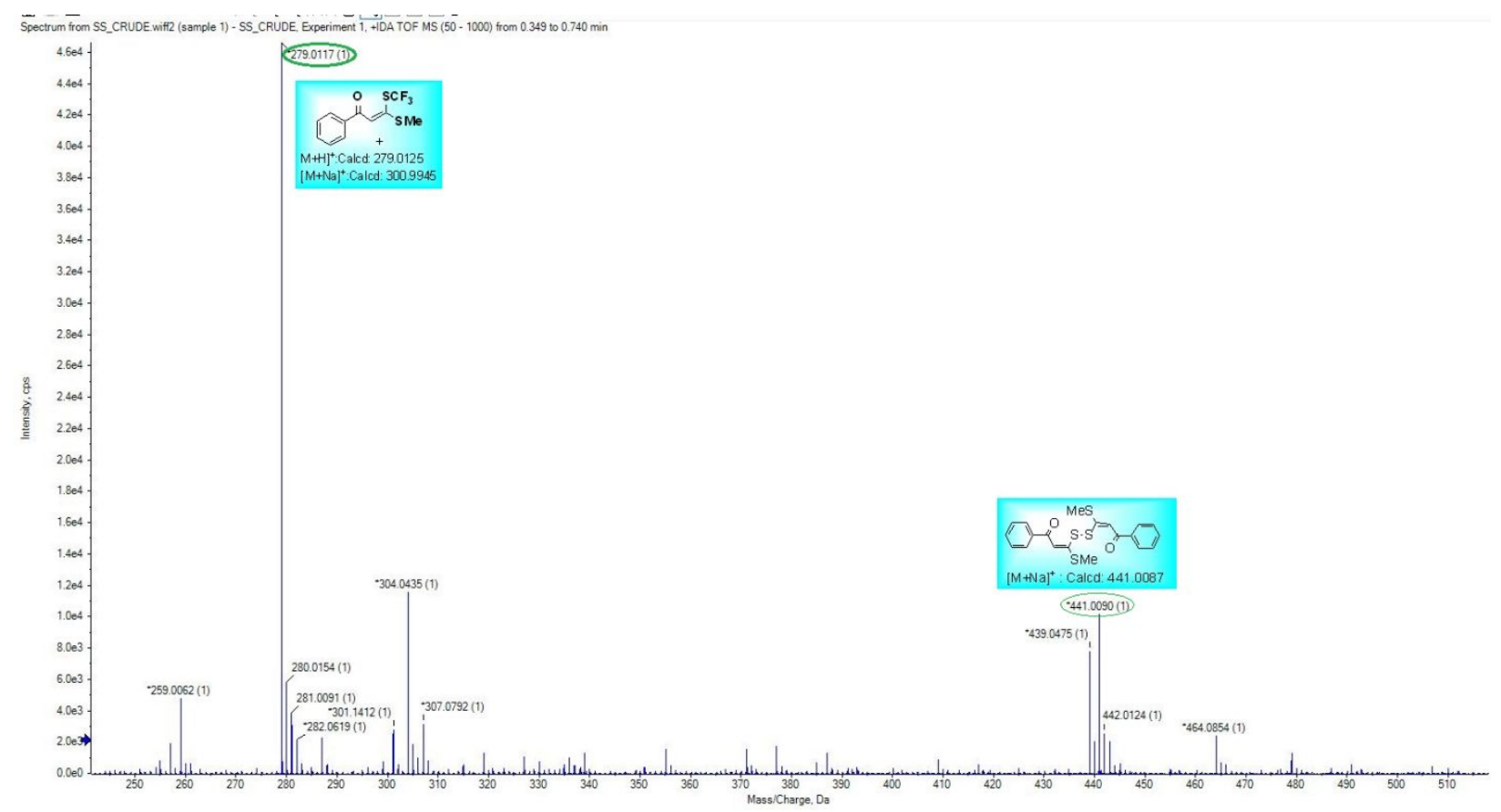

Figure S3. HRMS Spectra of Reaction Mixture Showing Peak for Intermediate Dimer B and Product formed $\mathbf{2 a}$ after $2 \mathrm{~h}$ of irradiation

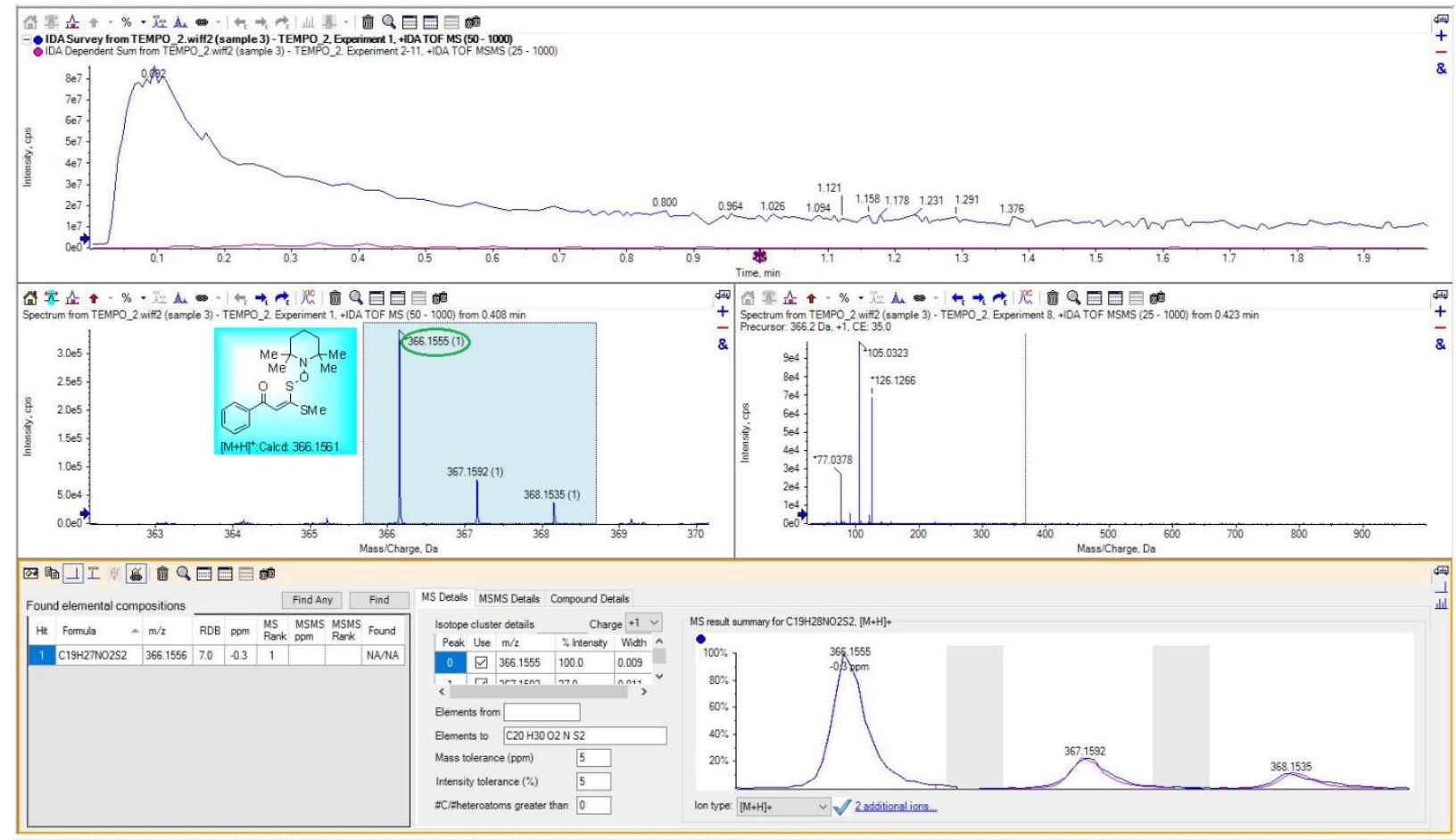

Figure S4. HRMS Spectra of Reaction Mixture Showing Peak for TEMPO-Adduct after $2 \mathrm{~h}$ of irradiation 


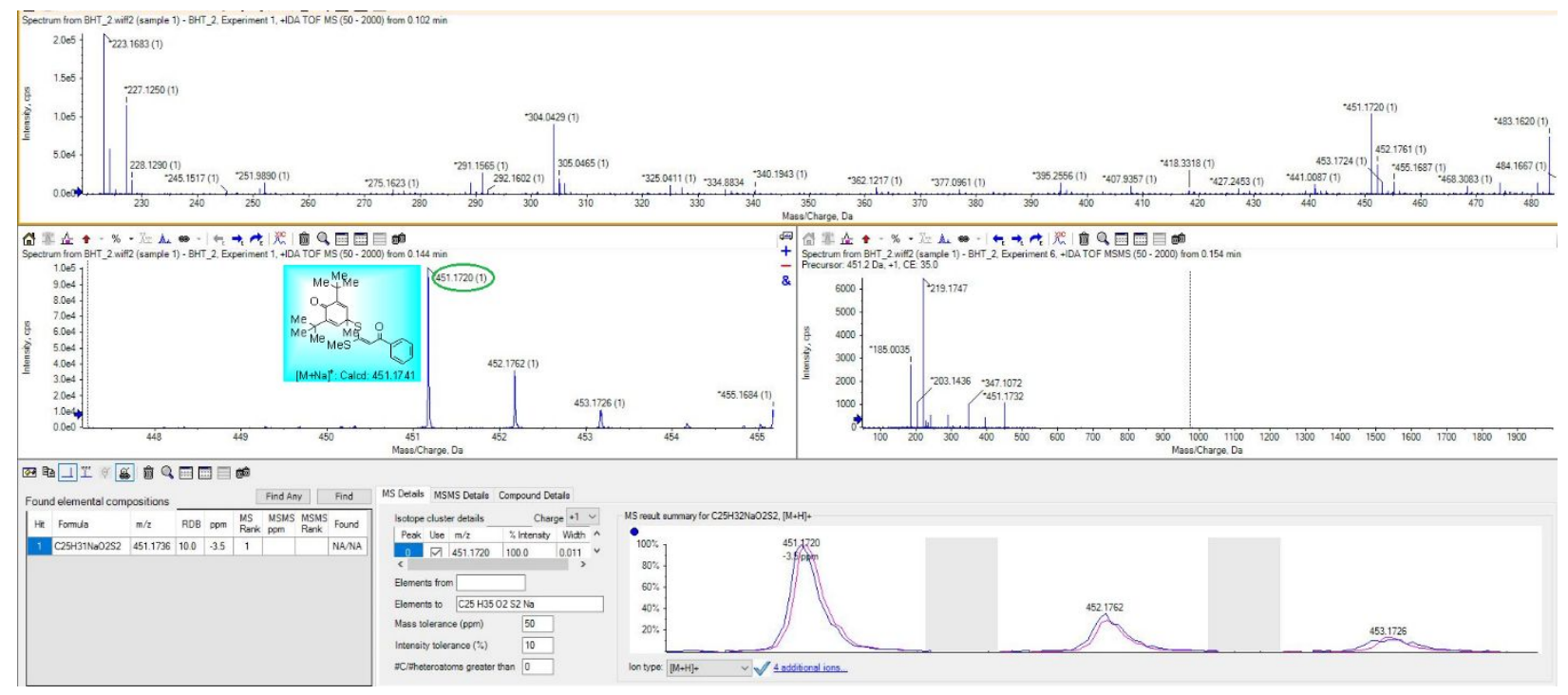

Figure S5. HRMS Spectra of Reaction Mixture Showing Peak for BHT-Adduct after 2 h of irradiation

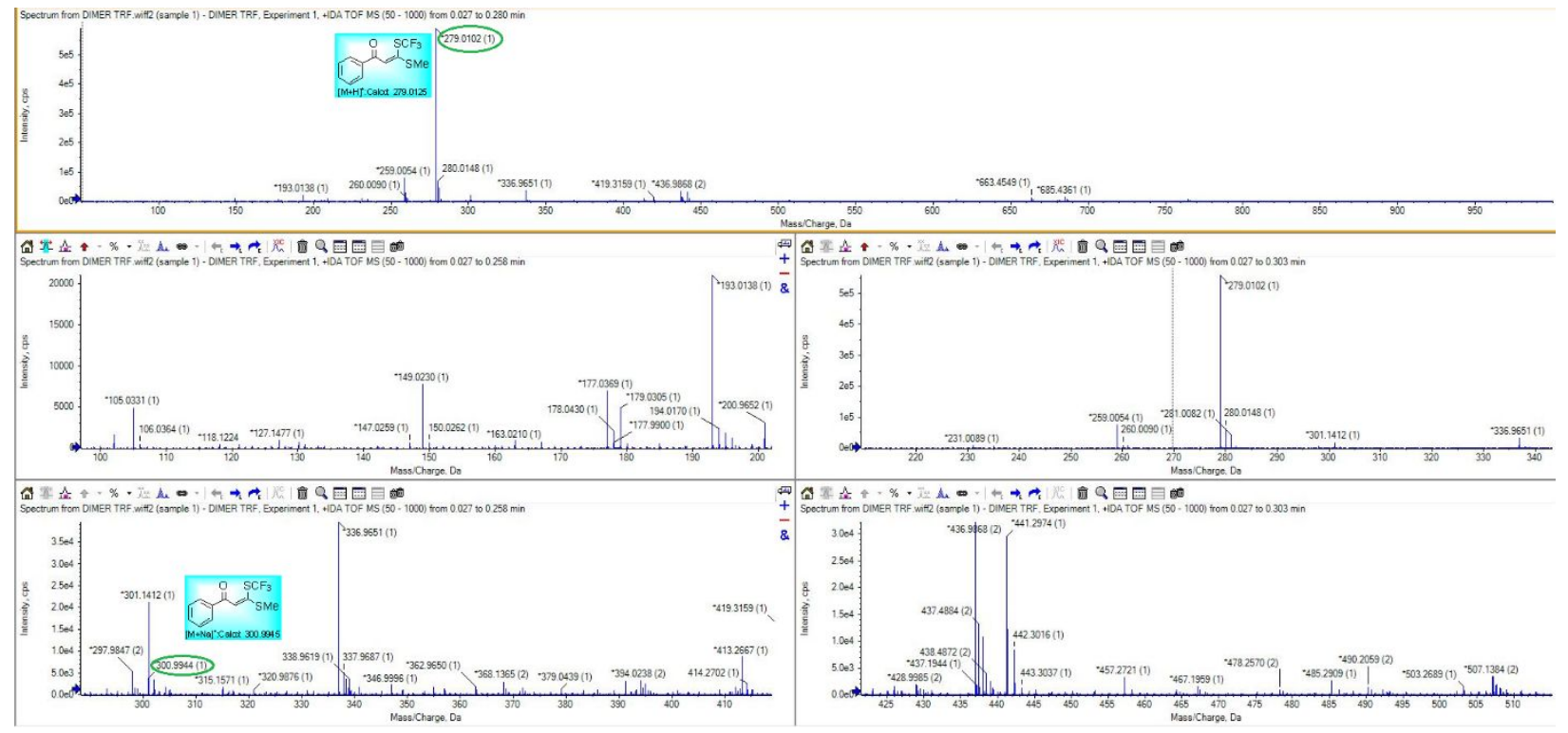

Figure S6. HRMS Spectra of Compound Obtained, 1a from Reaction of Dimer B under Optimized Condition after $15 \mathrm{~h}$ of irradiation 


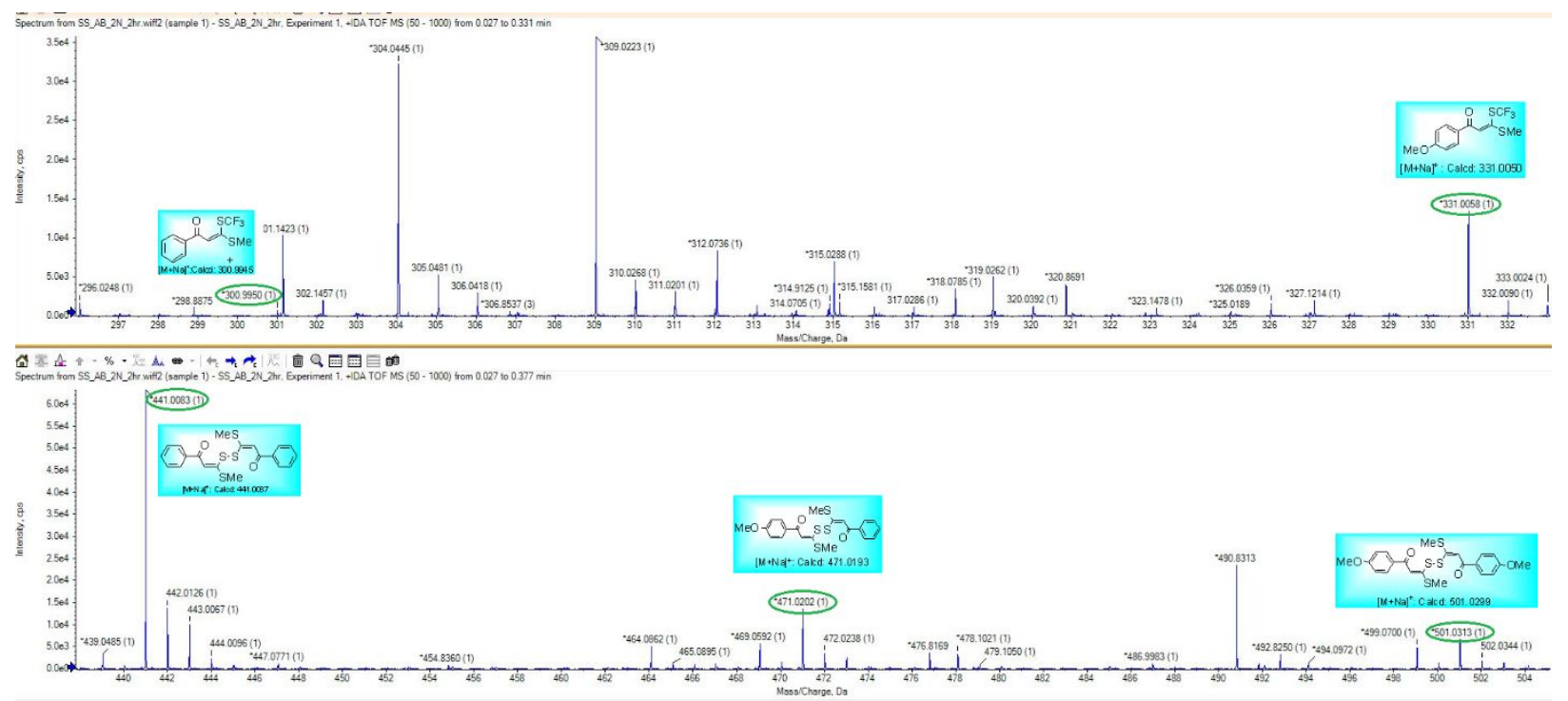

Figure S7. HRMS Spectra of Reaction Mixture of Cross-Reaction Performed between B and $\mathbf{1 j}$ after $2 \mathrm{~h}$ of irradiation

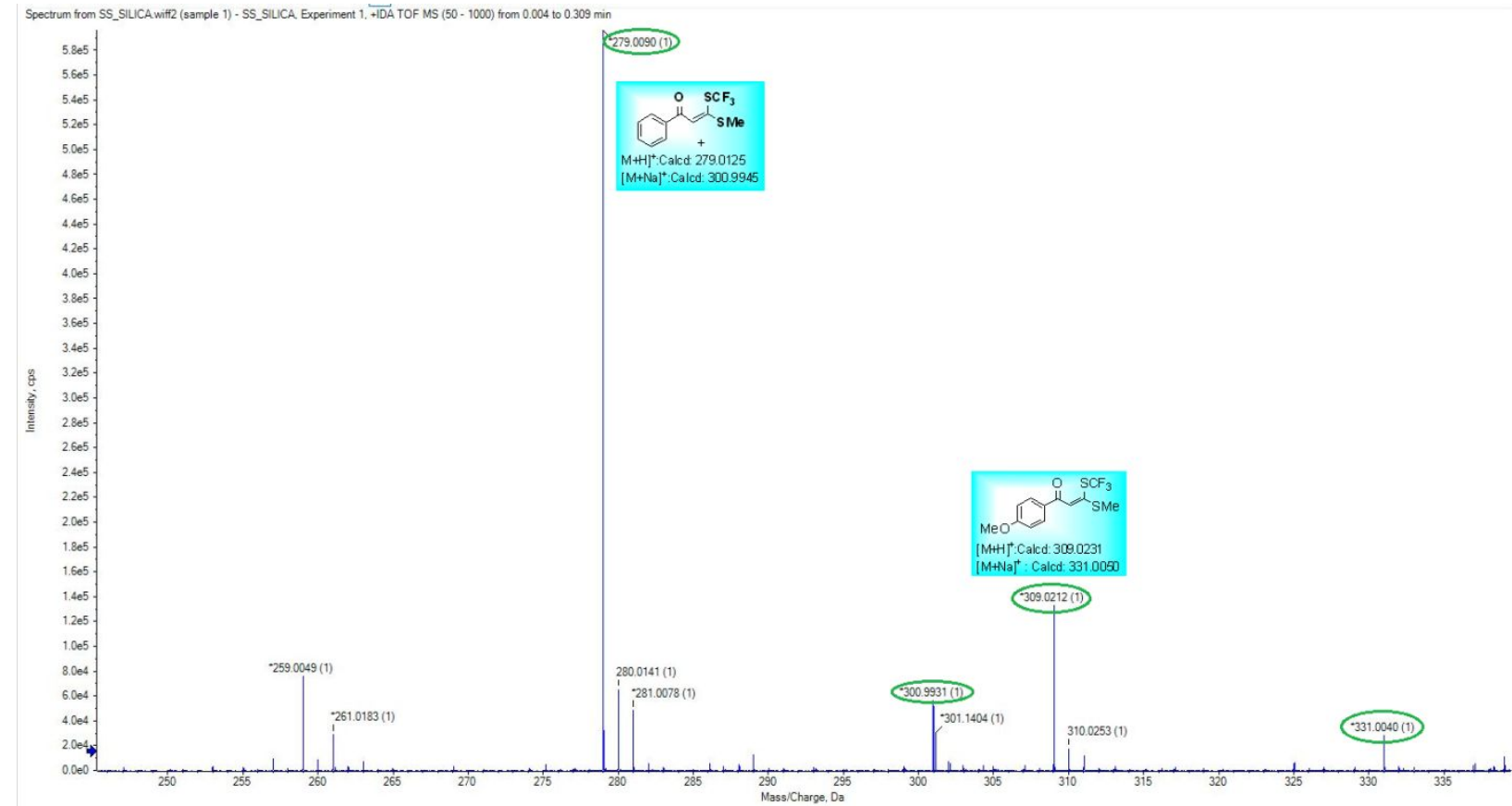

Figure S8. HRMS Spectra of Mixture of Isolated products i.e. 2a and $\mathbf{2 j}$ from the Cross-Reaction Performed between $\mathbf{B}$ and $\mathbf{1 j}$ after $15 \mathrm{~h}$ 
$\underline{{ }^{1} \mathrm{H} N M R,{ }^{13} \mathrm{C}\left\{{ }^{1} \mathrm{H}\right\} \text { NMR and }{ }^{19} \mathrm{~F} \text { NMR Spectra of Compound 2a }}$
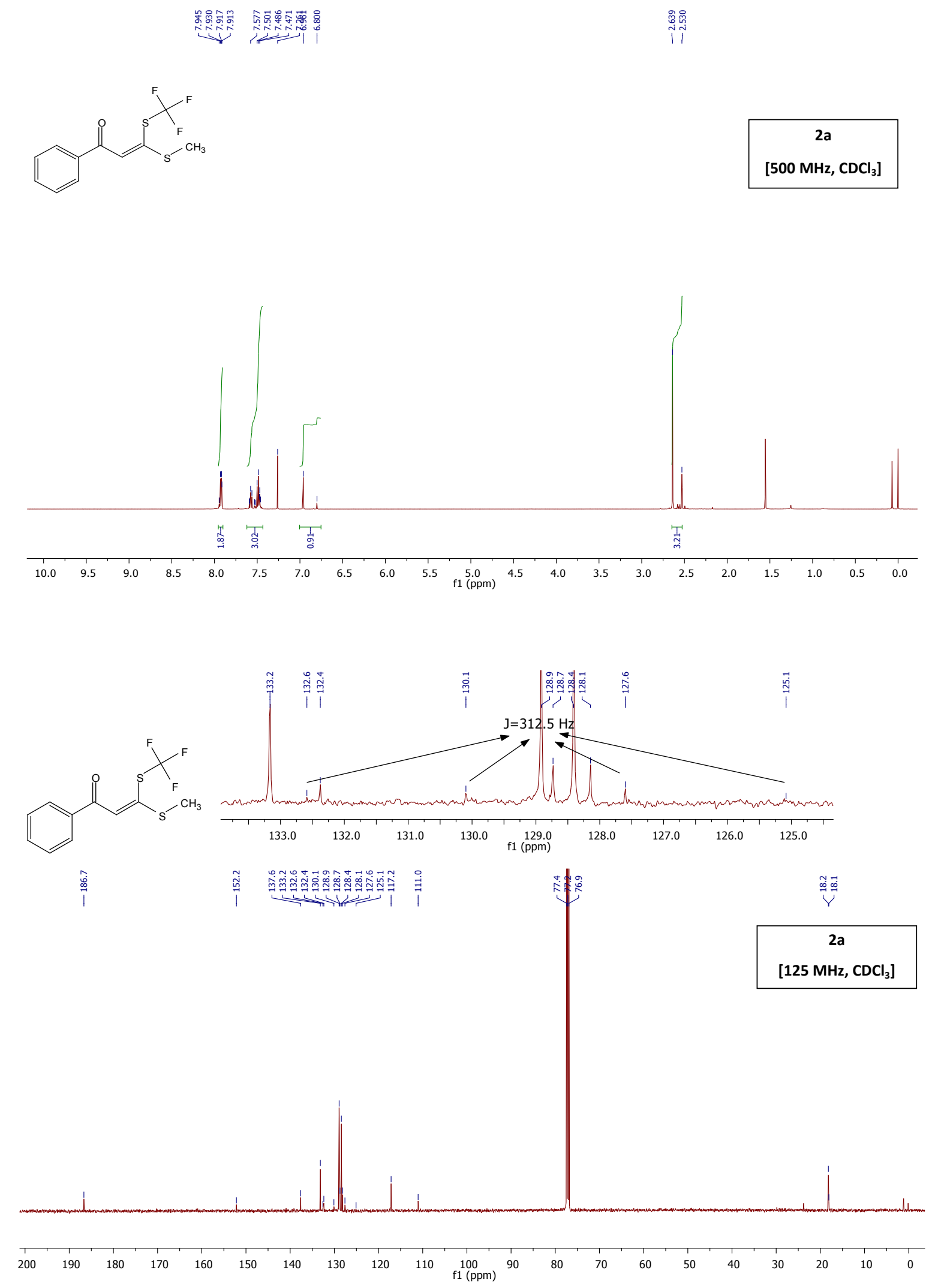


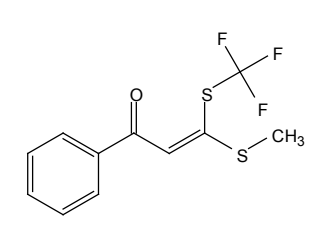

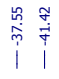

2a

[471 $\mathrm{MHz}, \mathrm{CDCl}_{3}$ ]



$\underline{{ }^{1} \mathrm{H} N M R,{ }^{13} \mathrm{C}\left\{{ }^{1} \mathrm{H}\right\} \text { NMR and }{ }^{19} \mathrm{~F} \text { NMR Spectra of Compound } \mathbf{2 b}}$


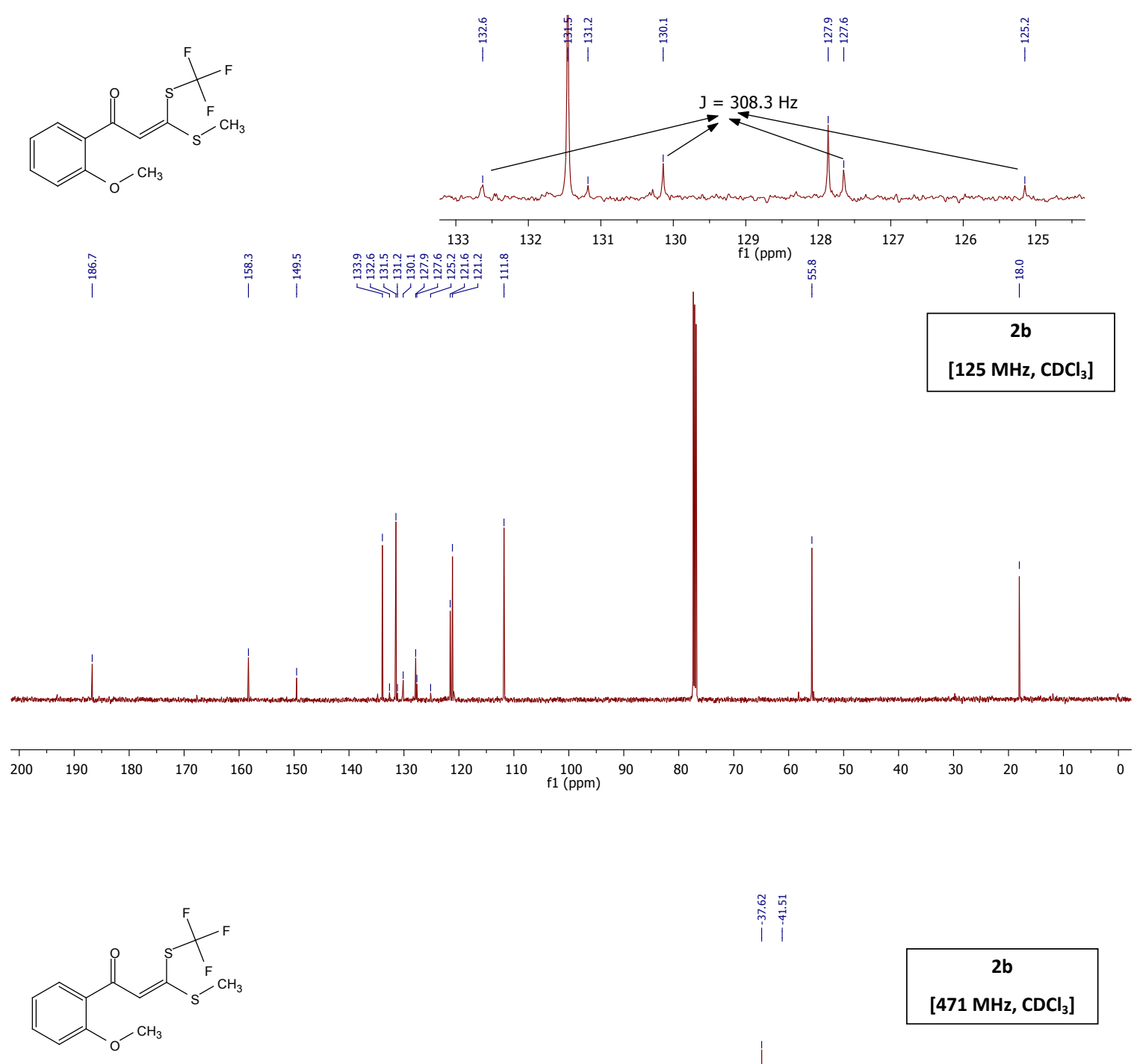

产

$2 b$

[471 $\mathrm{MHz}, \mathrm{CDCl}_{3}$ ]

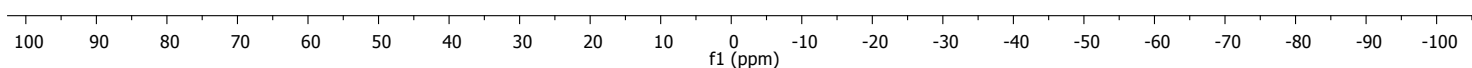


$\underline{{ }^{1} \mathrm{H} N M R,{ }^{13} \mathrm{C}\left\{{ }^{1} \mathrm{H}\right\} \text { NMR and }{ }^{19} \mathrm{~F} \text { NMR Spectra of Compound } \mathbf{2 c}}$


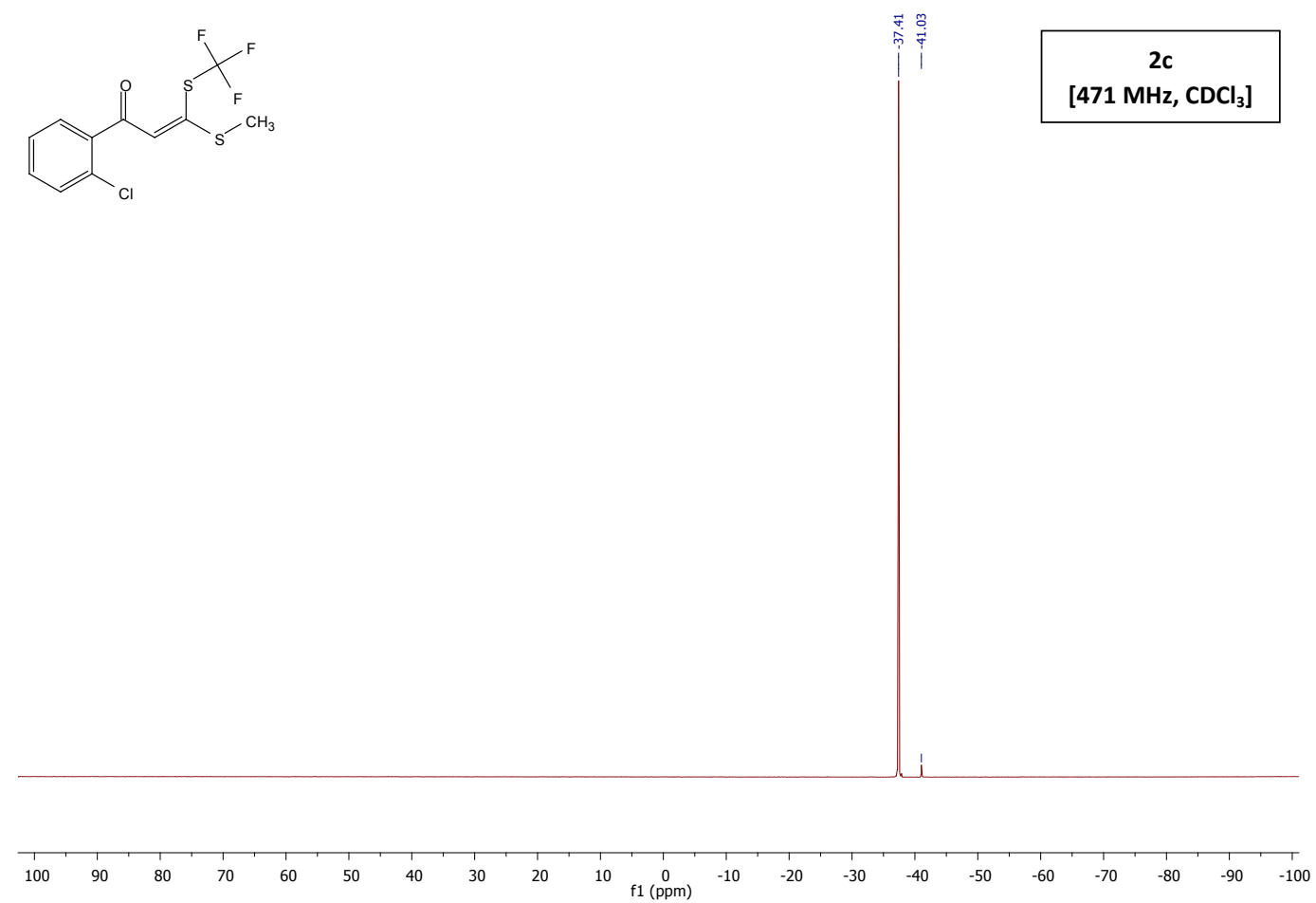

\section{$\underline{{ }^{1} \mathrm{H} N M R,{ }^{13} \mathrm{C}\left\{{ }^{1} \mathrm{H}\right\} \text { NMR and }{ }^{19} \mathrm{~F} \text { NMR Spectra of Compound } \mathbf{2 d}}$}
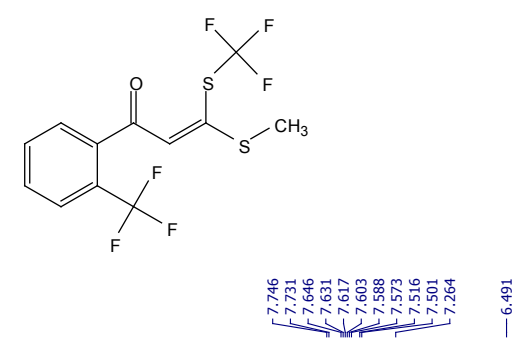

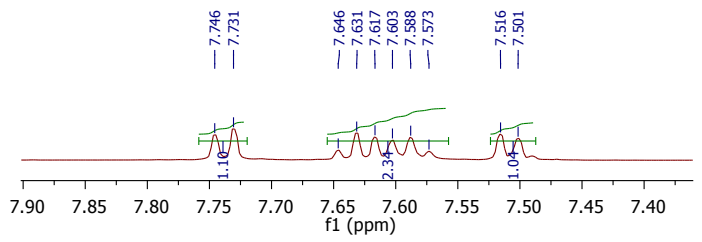

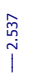

2d $\left[500 \mathrm{MHz}, \mathrm{CDCl}_{3}\right]$

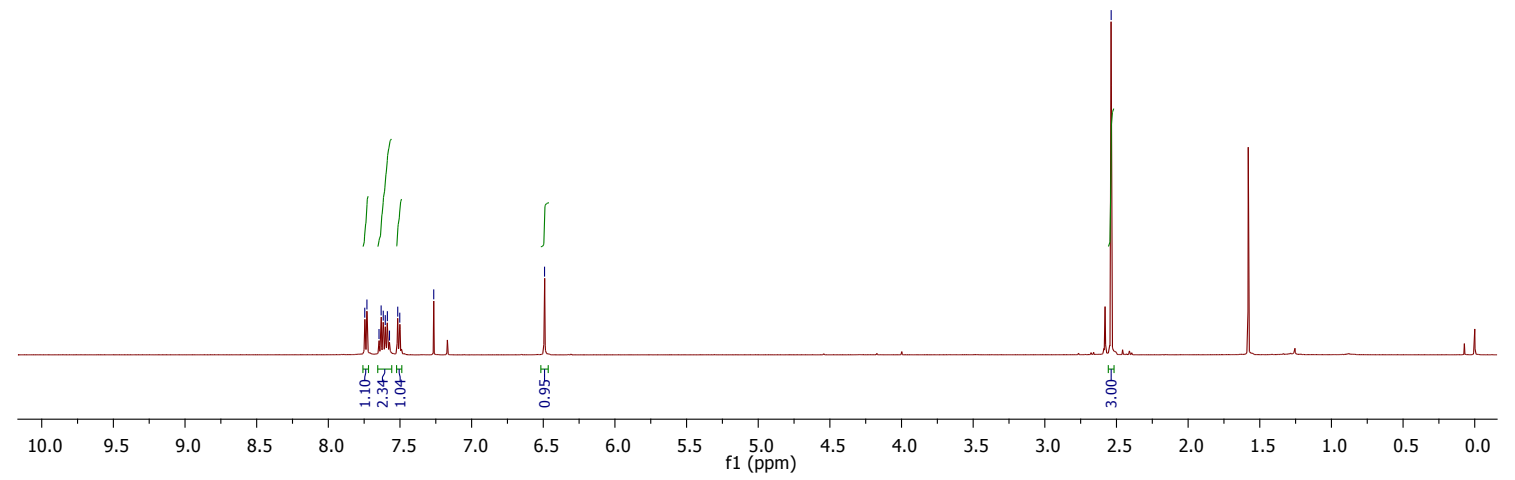





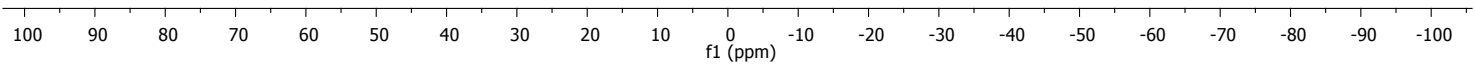


$\underline{{ }^{1} \mathrm{H} N M R,{ }^{13} \mathrm{C}\left\{{ }^{1} \mathrm{H}\right\} \text { NMR and }{ }^{19} \mathrm{~F} \text { NMR Spectra of Compound } \mathbf{2 e}}$
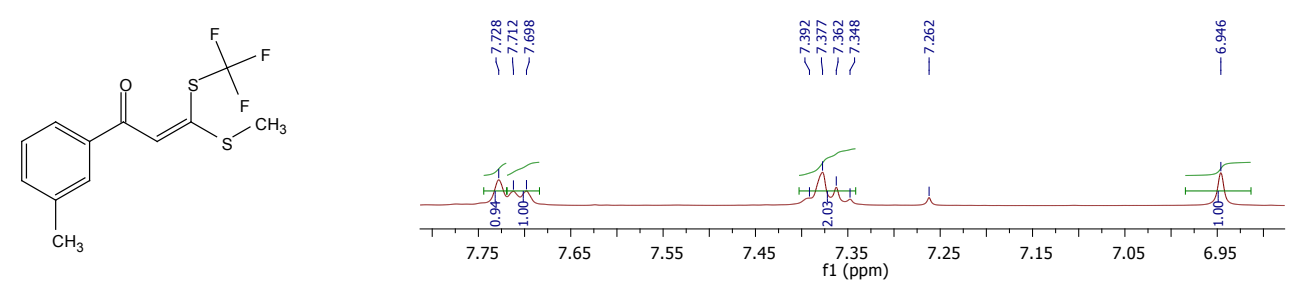

|

|

$2 \mathrm{e}$

[500 MHz, $\mathrm{CDCl}_{3}$ ]
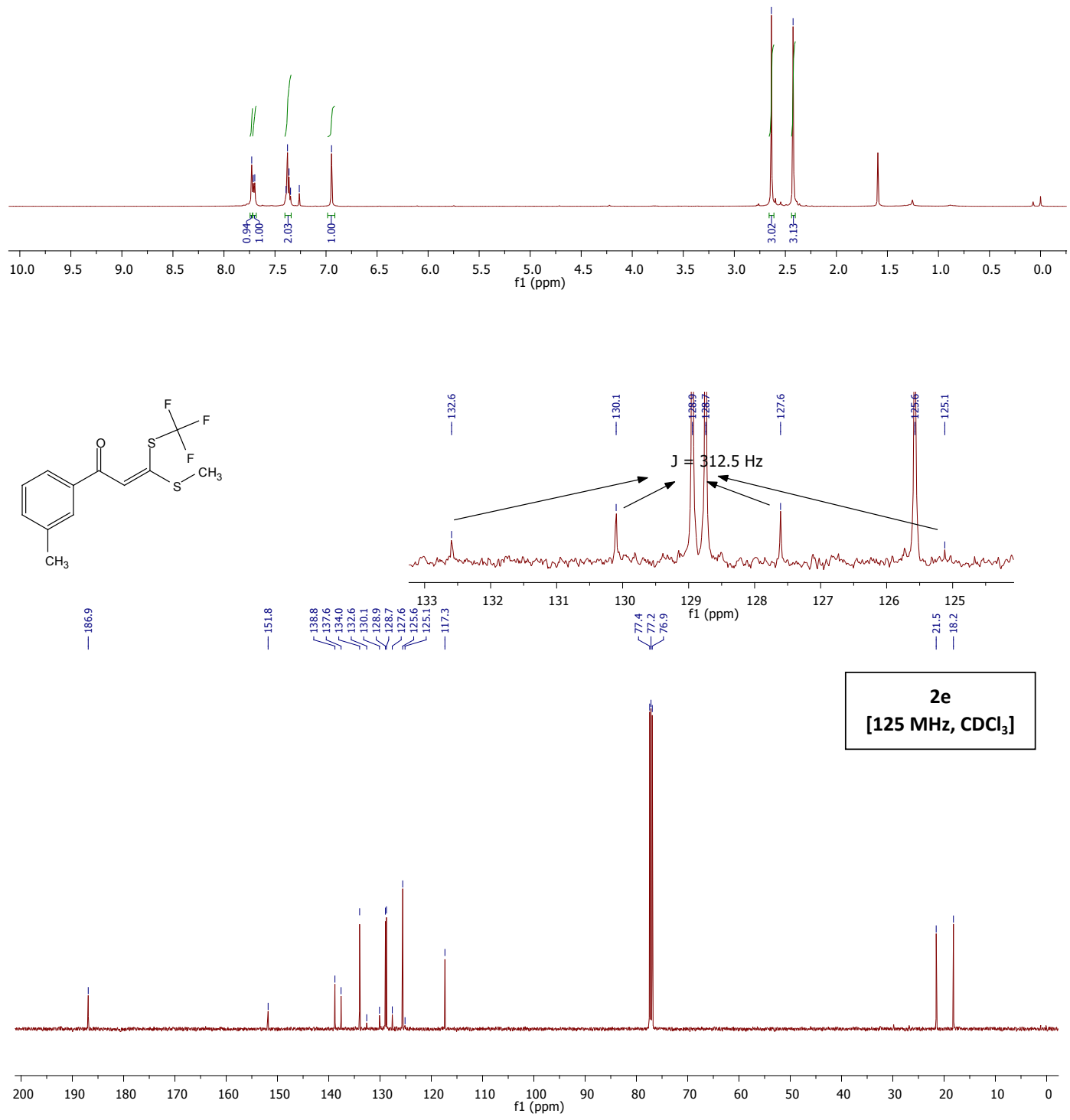



$\underline{{ }^{1} \mathrm{H} N M R,{ }^{13} \mathrm{C}\left\{{ }^{1} \mathrm{H}\right\} \text { NMR and }{ }^{19} \mathrm{~F} \text { NMR Spectra of Compound } \mathbf{2 f}}$

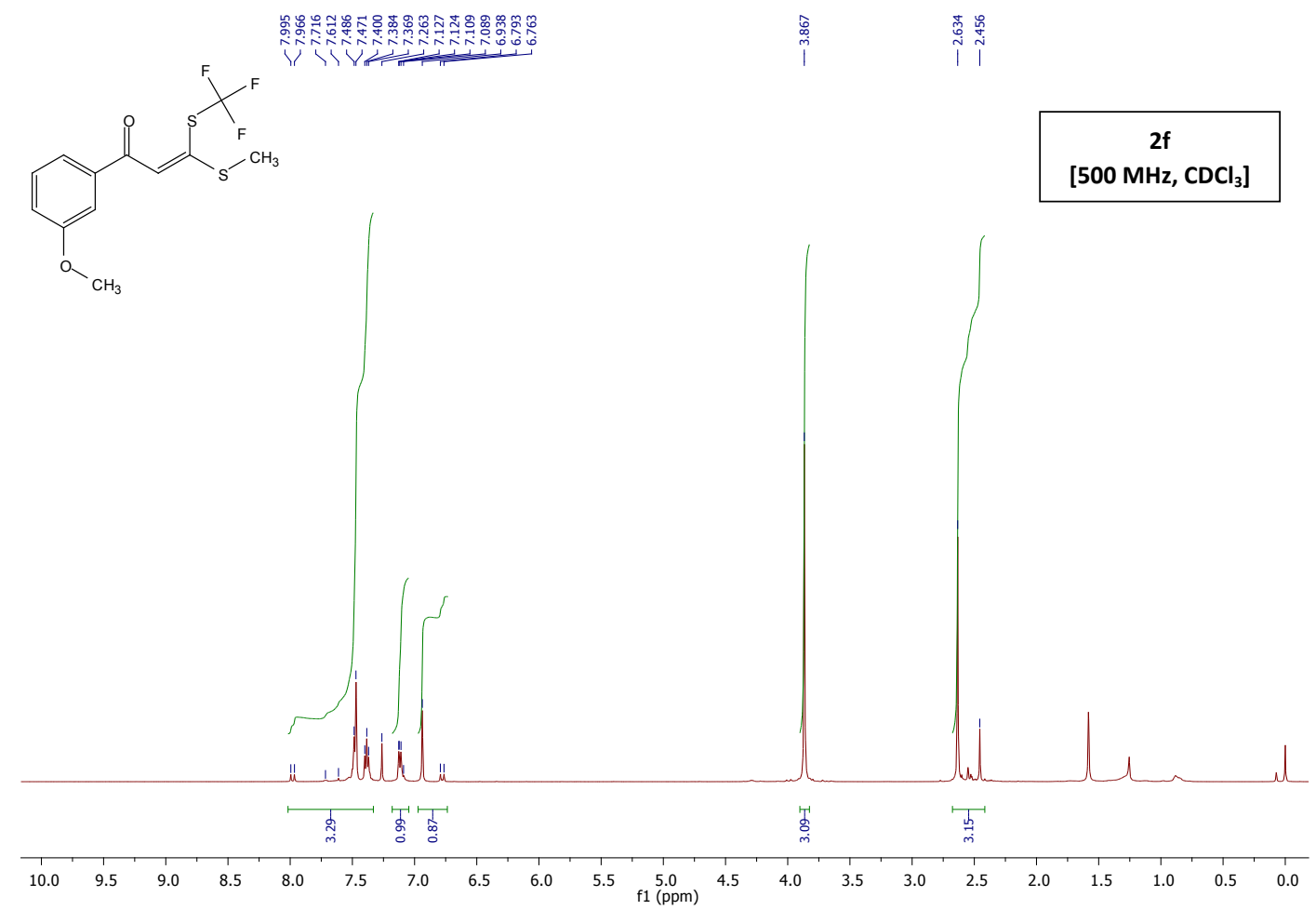



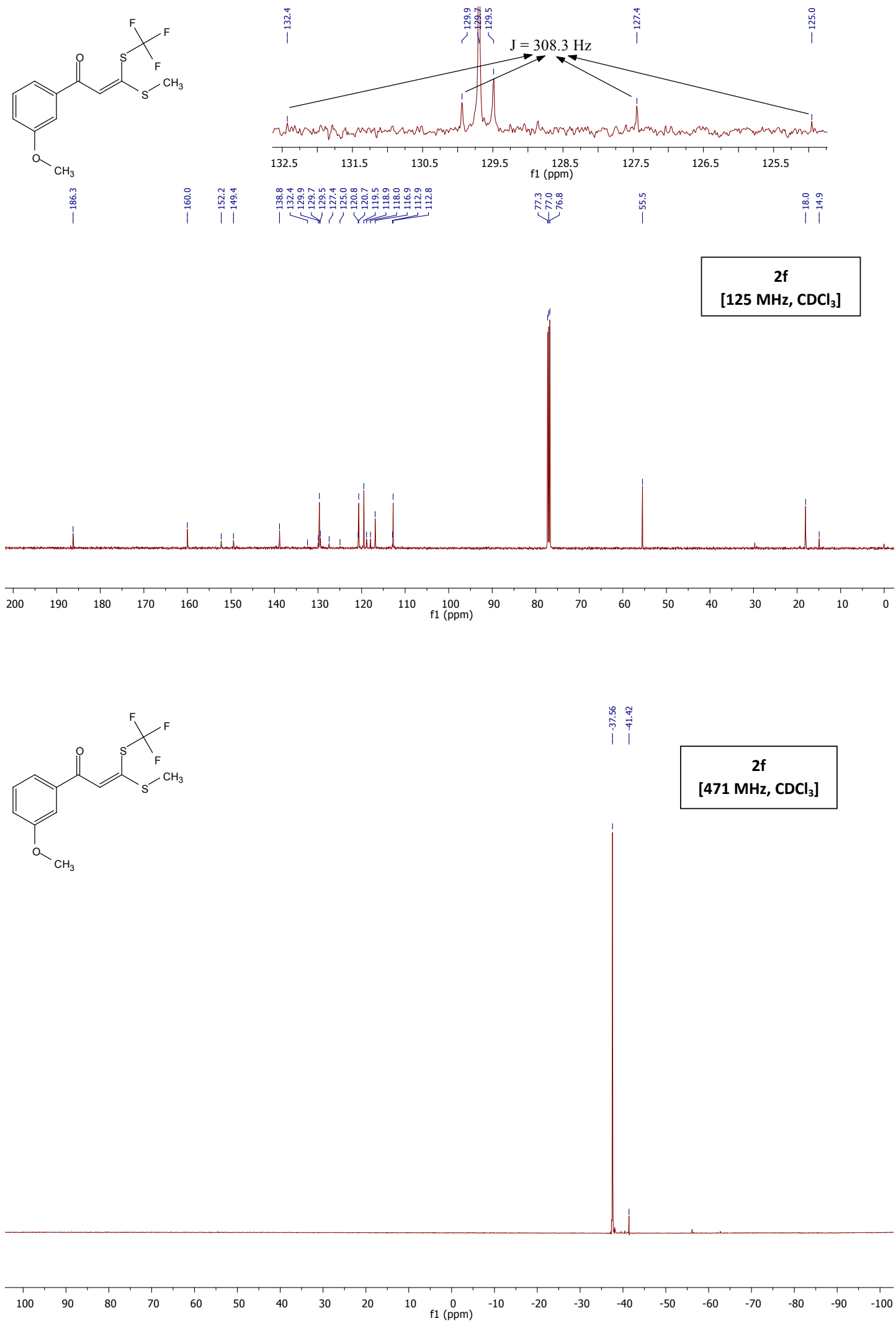
$\underline{{ }^{1} \mathrm{H} N M R,{ }^{13} \mathrm{C}\left\{{ }^{1} \mathrm{H}\right\} \text { NMR and }{ }^{19} \mathrm{~F} \text { NMR Spectra of Compound } \mathbf{2 g}}$<smiles>CSC(=CC(=O)c1cccc(Cl)c1)SC(F)(F)F</smiles>
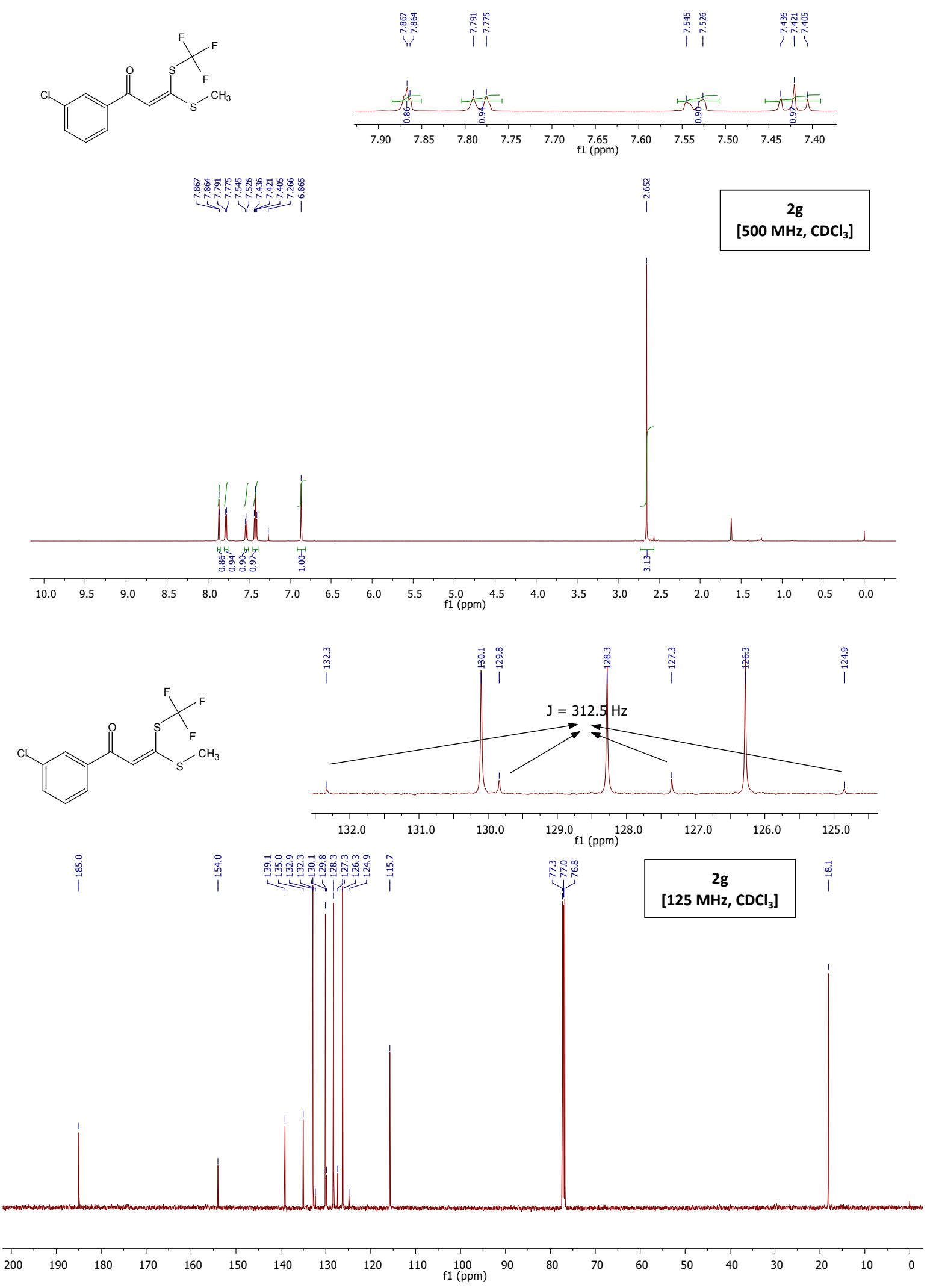

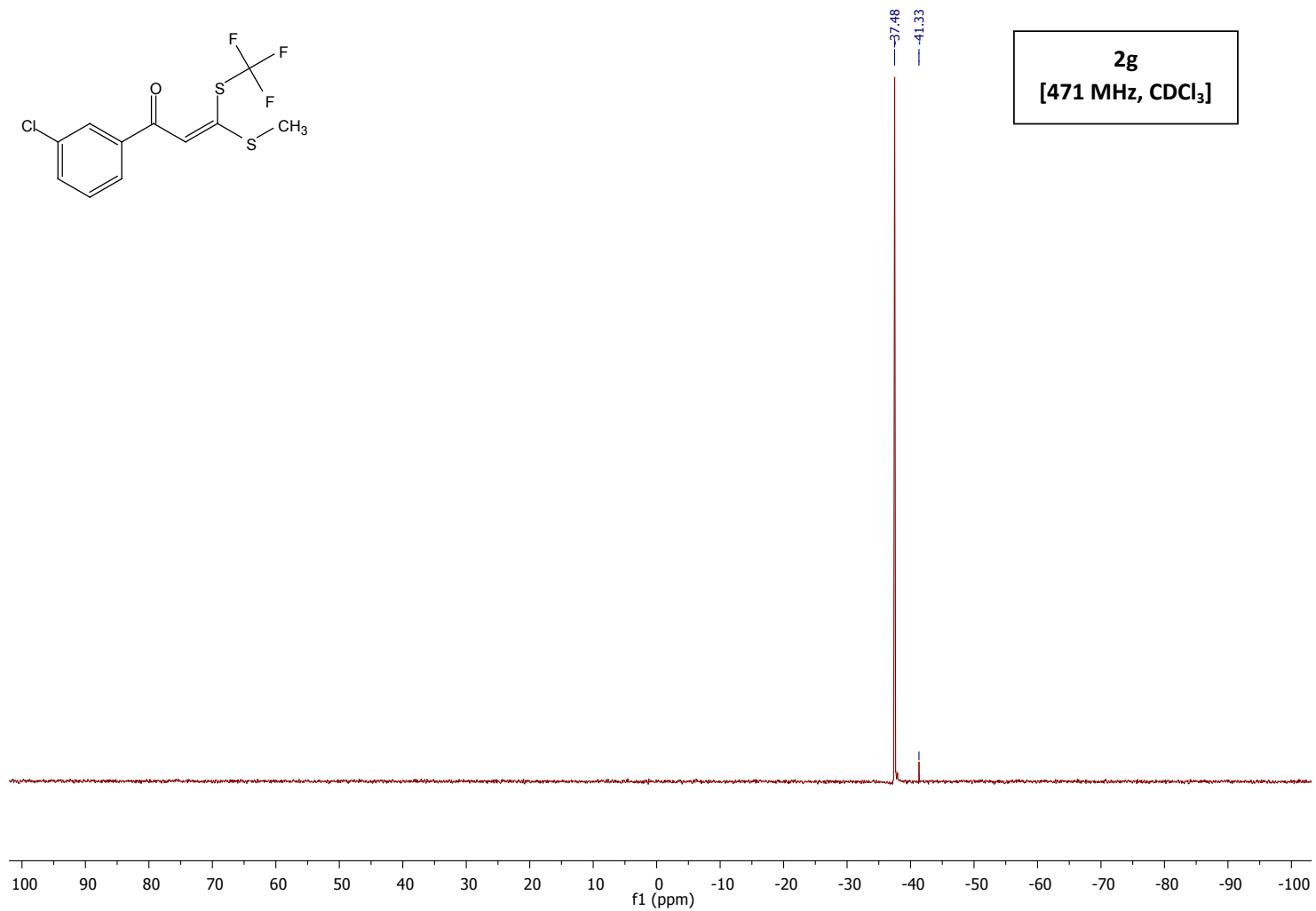

$\underline{{ }^{1} \mathrm{H} N M R,{ }^{13} \mathrm{C}\left\{{ }^{1} \mathrm{H}\right\} \text { NMR and }{ }^{19} \mathrm{~F} \text { NMR Spectra of Compound } \mathbf{2 h}}$
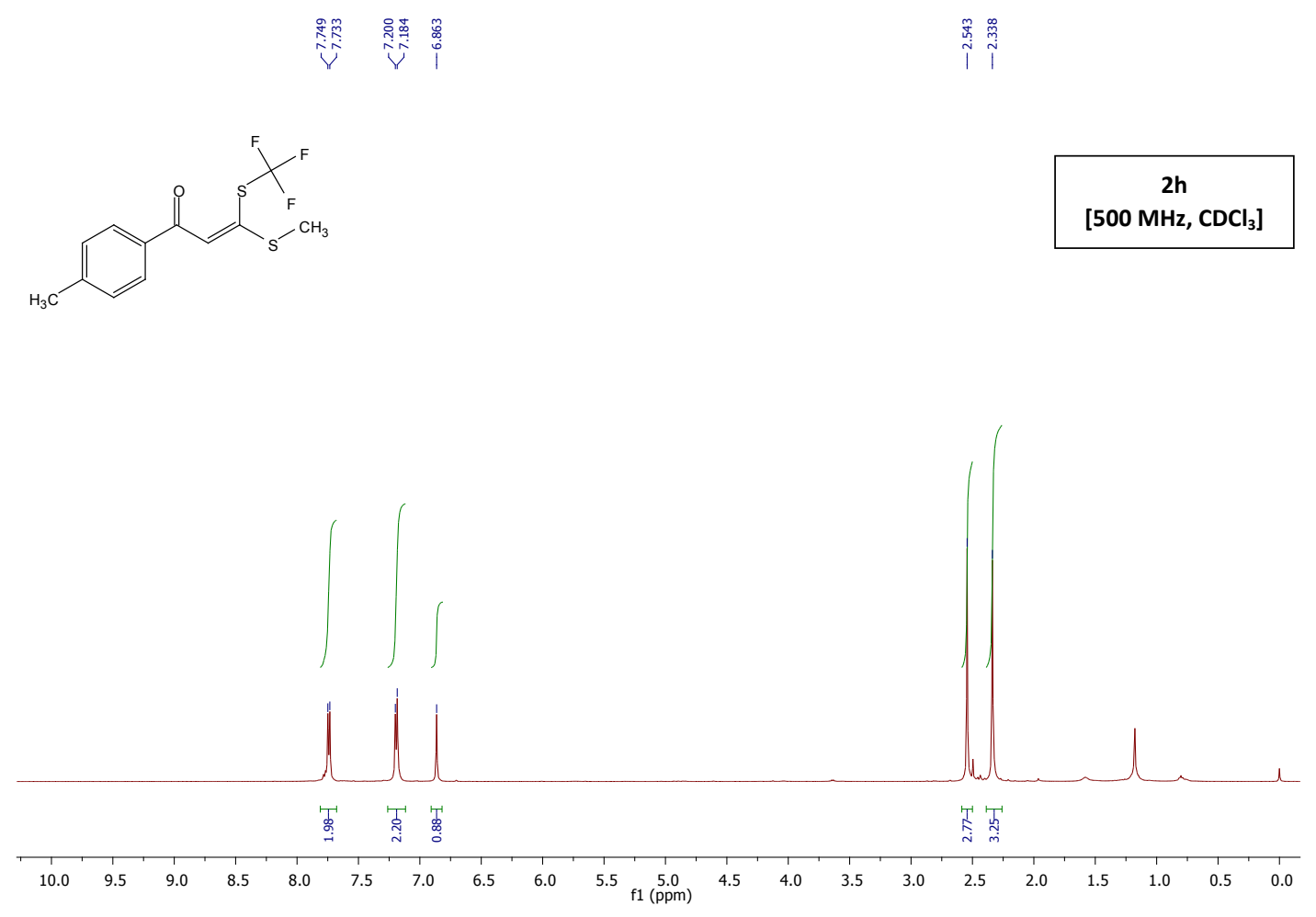

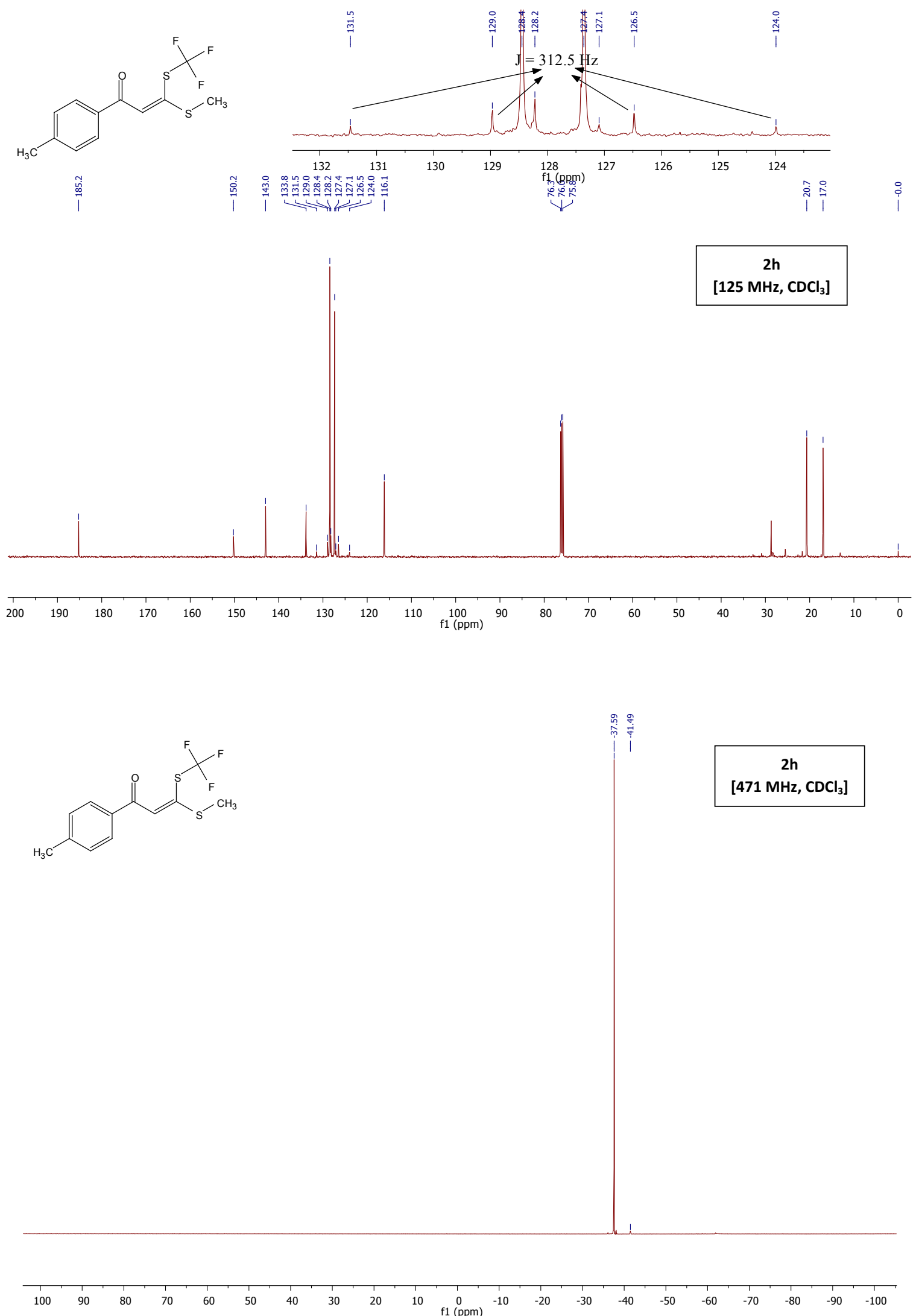
$\underline{{ }^{1} \mathrm{H} N M R,{ }^{13} \mathrm{C}\left\{{ }^{1} \mathrm{H}\right\} \text { NMR and }{ }^{19} \mathrm{~F} \text { NMR Spectra of Compound } \mathbf{2 i}}$
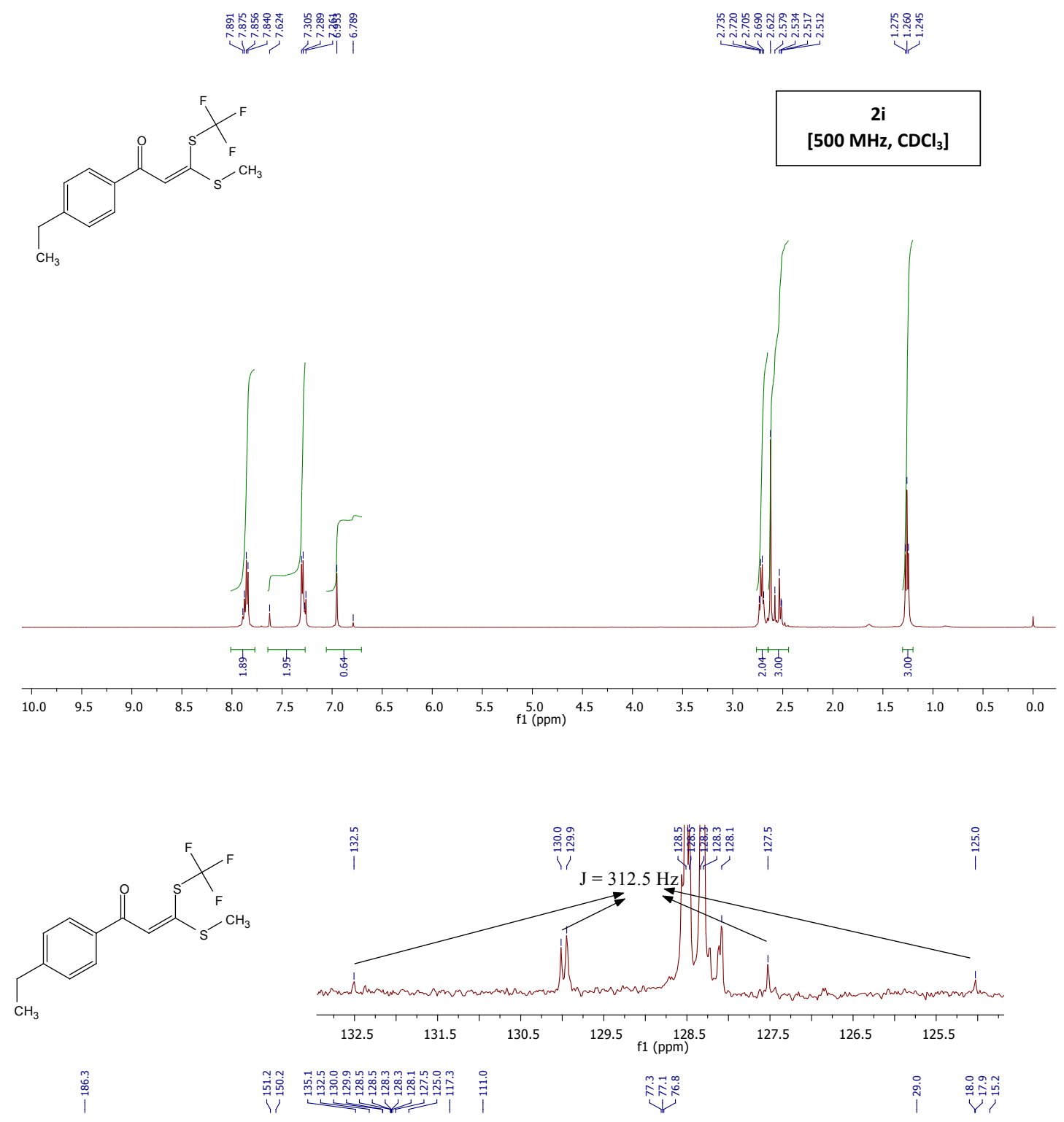

$\sqrt{n+\infty}$

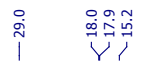



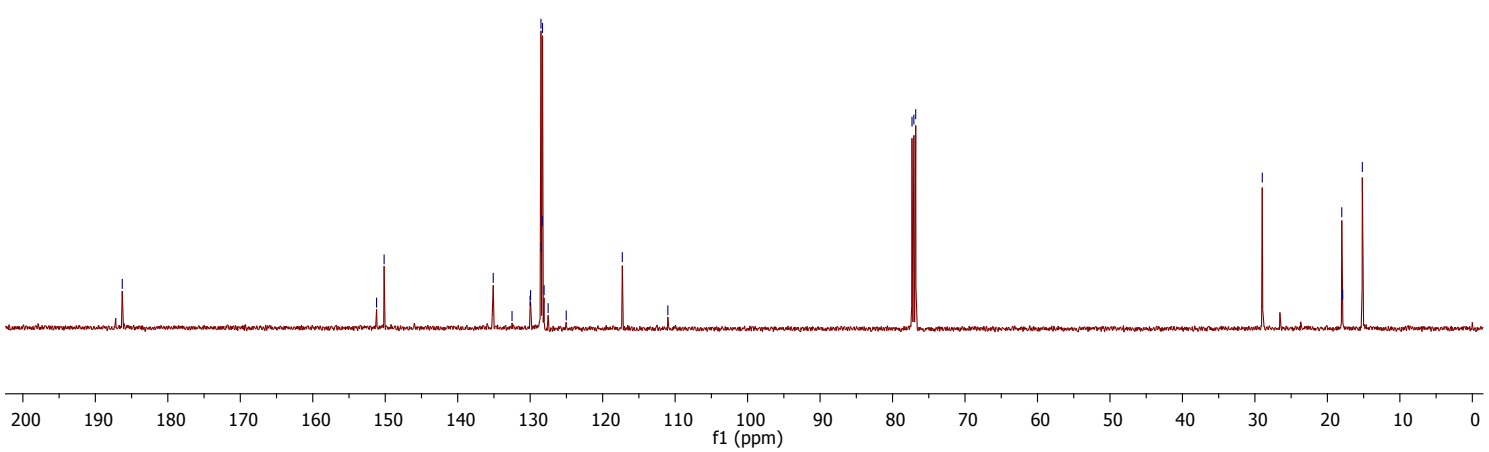



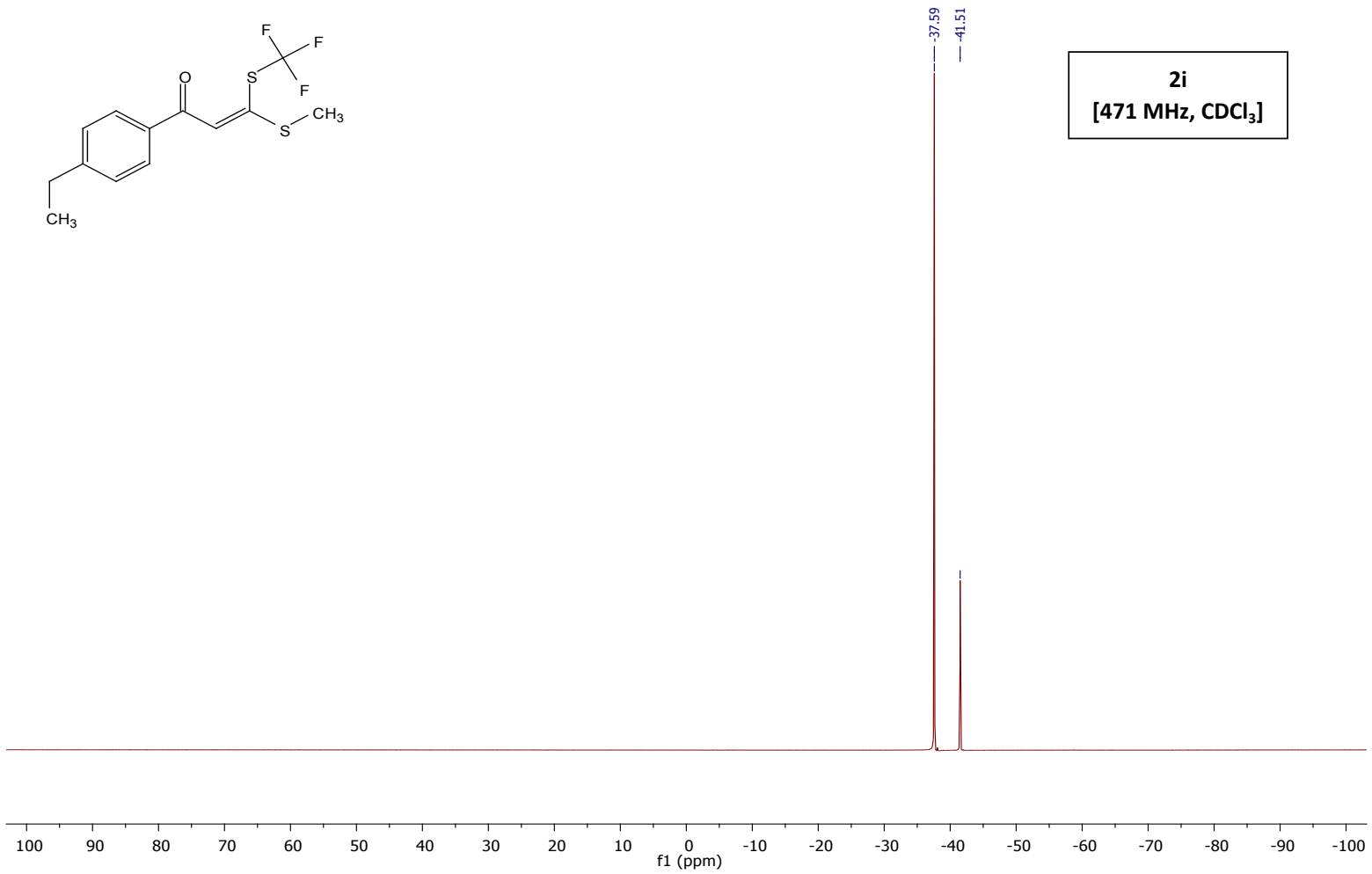

$\underline{{ }^{1} \mathrm{H} \text { NMR, }{ }^{13} \mathrm{C}\left\{{ }^{1} \mathrm{H}\right\} \text { NMR and }{ }^{19} \mathrm{~F} \text { NMR Spectra of Compound } \mathbf{2} \mathbf{j}}$





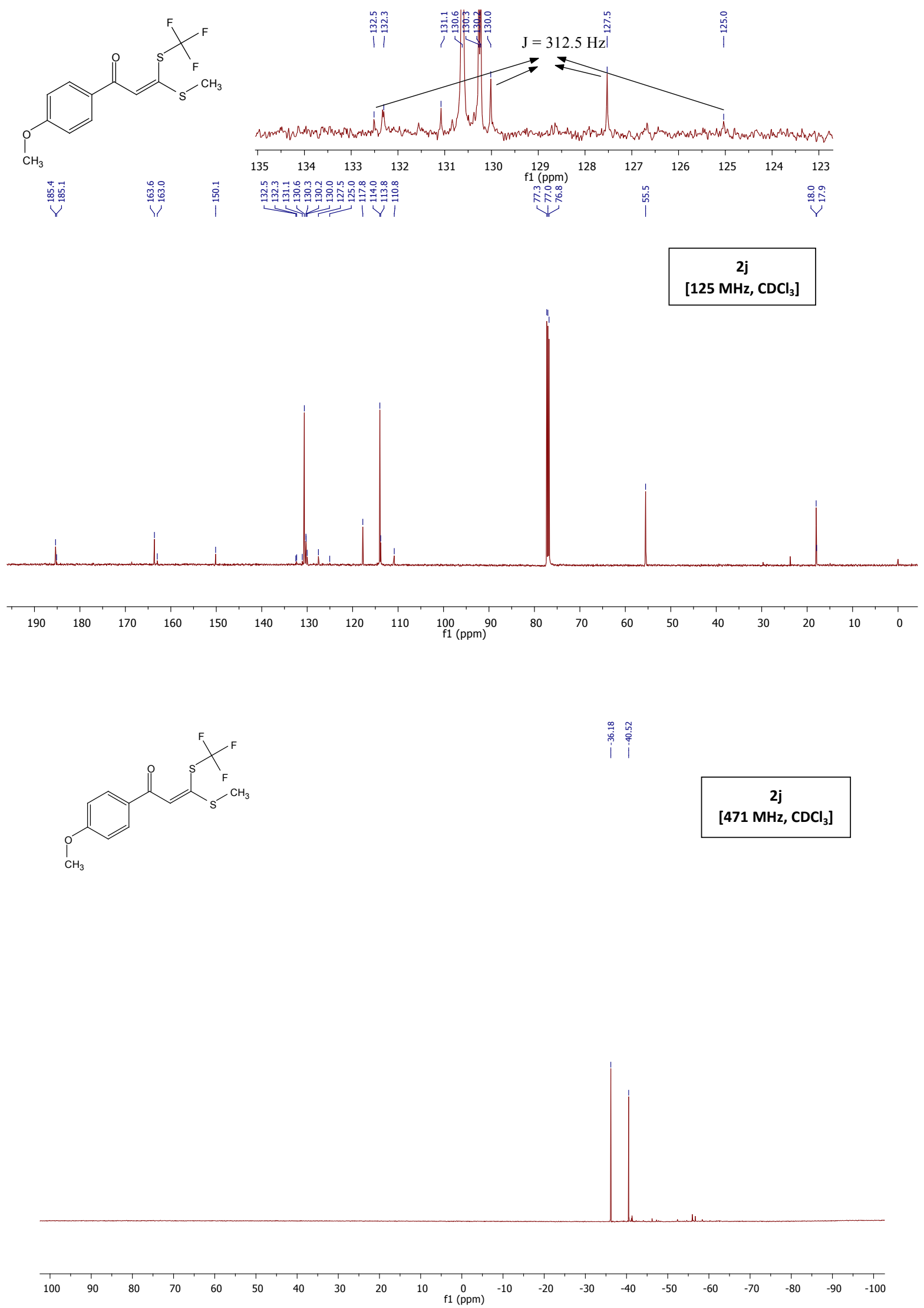
$\underline{{ }^{1} \mathrm{H} N M R,{ }^{13} \mathrm{C}\left\{{ }^{1} \mathrm{H}\right\} \text { NMR and }{ }^{19} \mathrm{~F} \text { NMR Spectra of Compound } \mathbf{2 k}}$
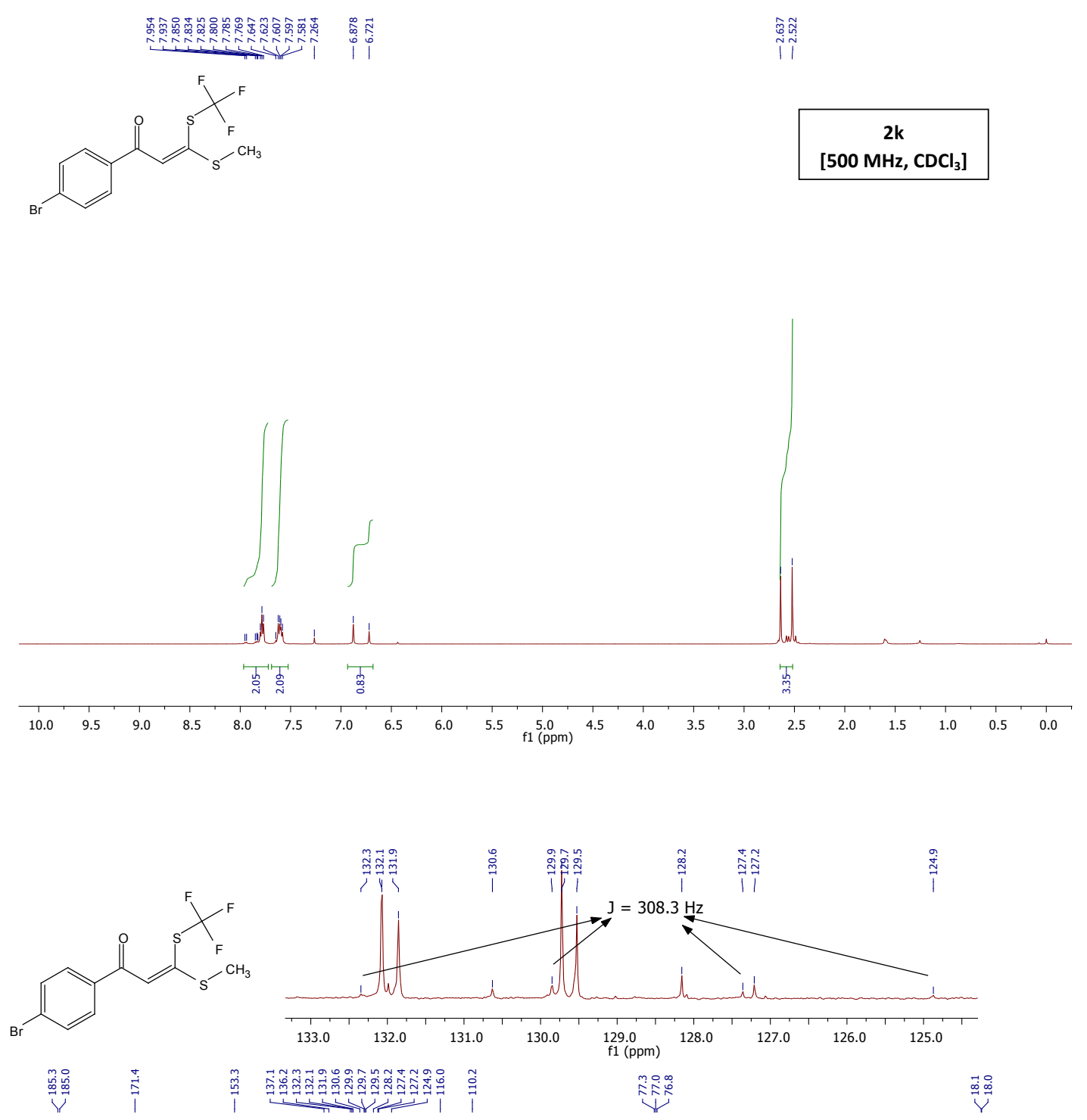

$2 \mathbf{k}$ [125 $\mathrm{MHz}^{\mathrm{CDCl}} \mathrm{CDC}_{3}$ ]





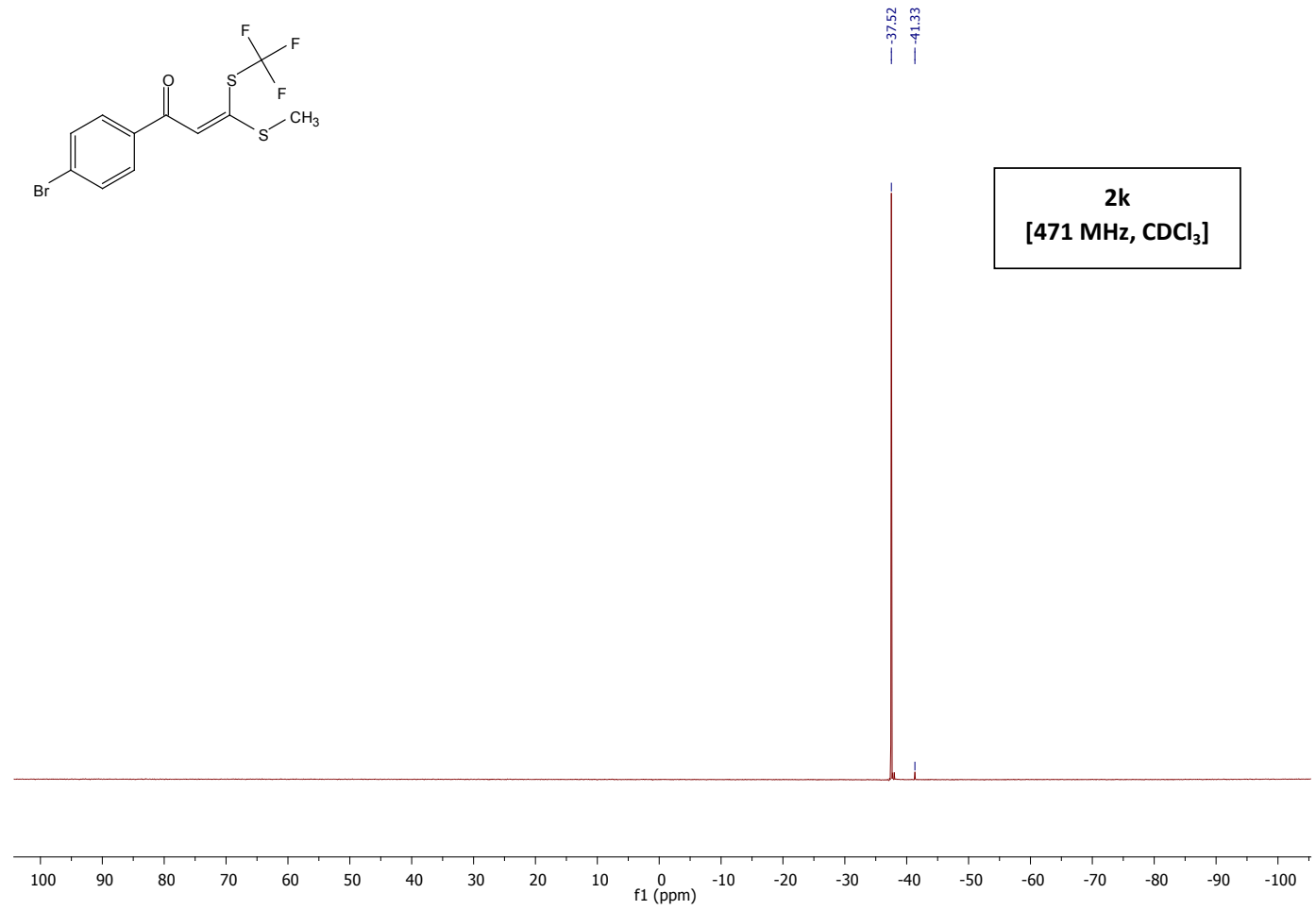

$\underline{{ }^{1} \mathrm{H} \text { NMR, }{ }^{13} \mathrm{C}\left\{{ }^{1} \mathrm{H}\right\} \text { NMR and }{ }^{19} \mathrm{~F} \text { NMR Spectra of Compound } \mathbf{2 l}}$

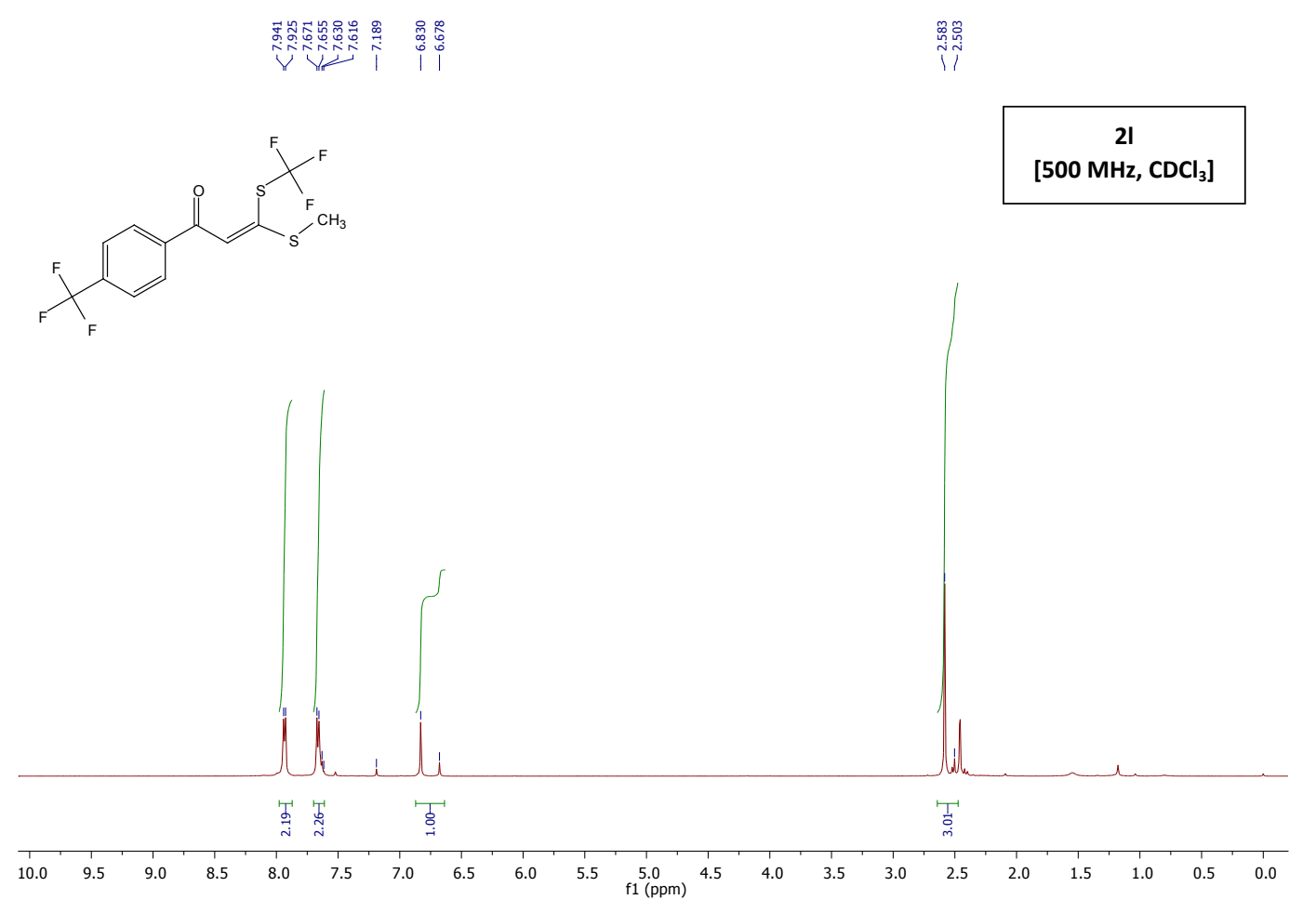



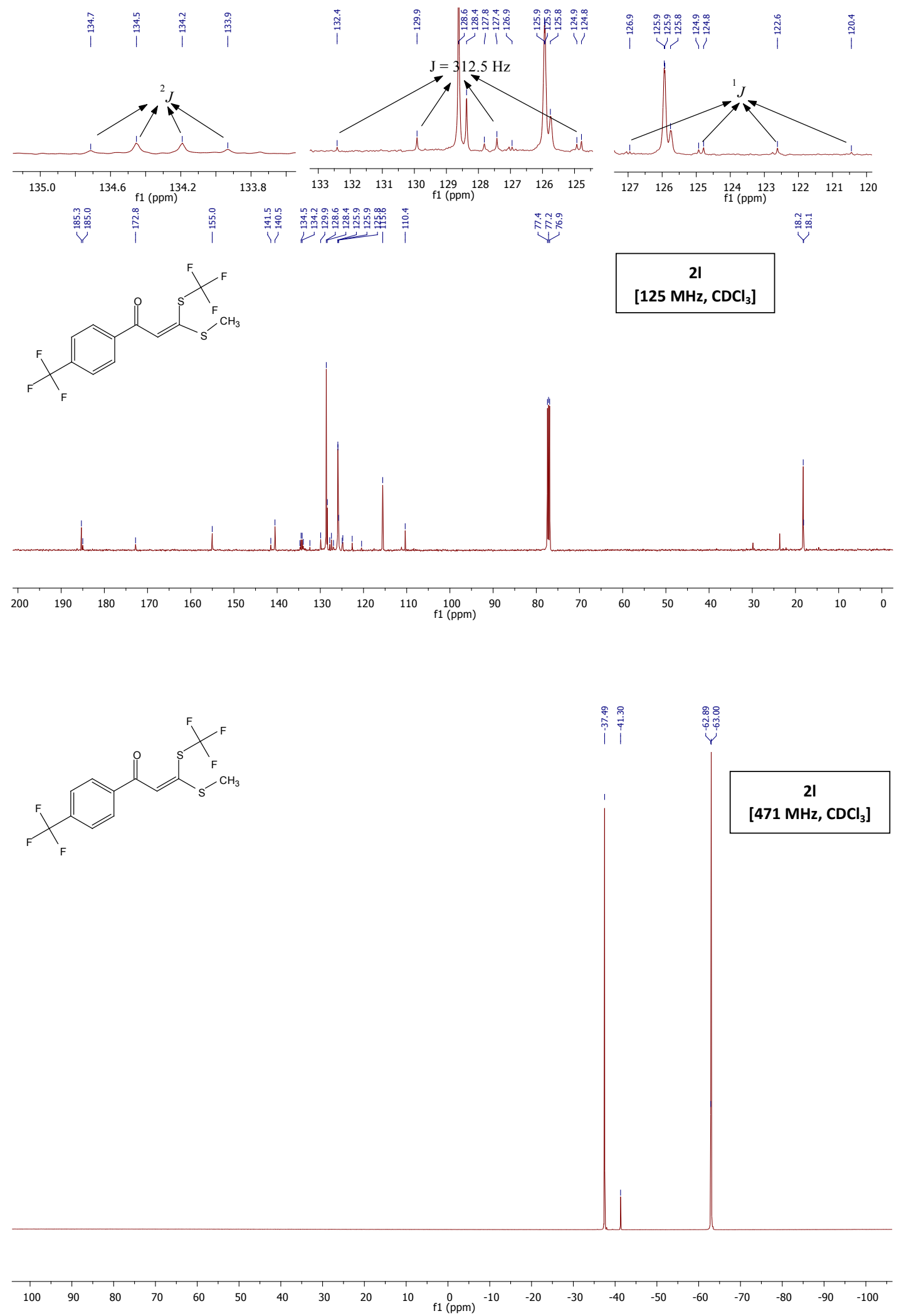
$\underline{{ }^{1} \mathrm{H} N M R,{ }^{13} \mathrm{C}\left\{{ }^{1} \mathrm{H}\right\} \text { NMR and }{ }^{19} \mathrm{~F} \text { NMR Spectra of Compound } \mathbf{2 m}}$


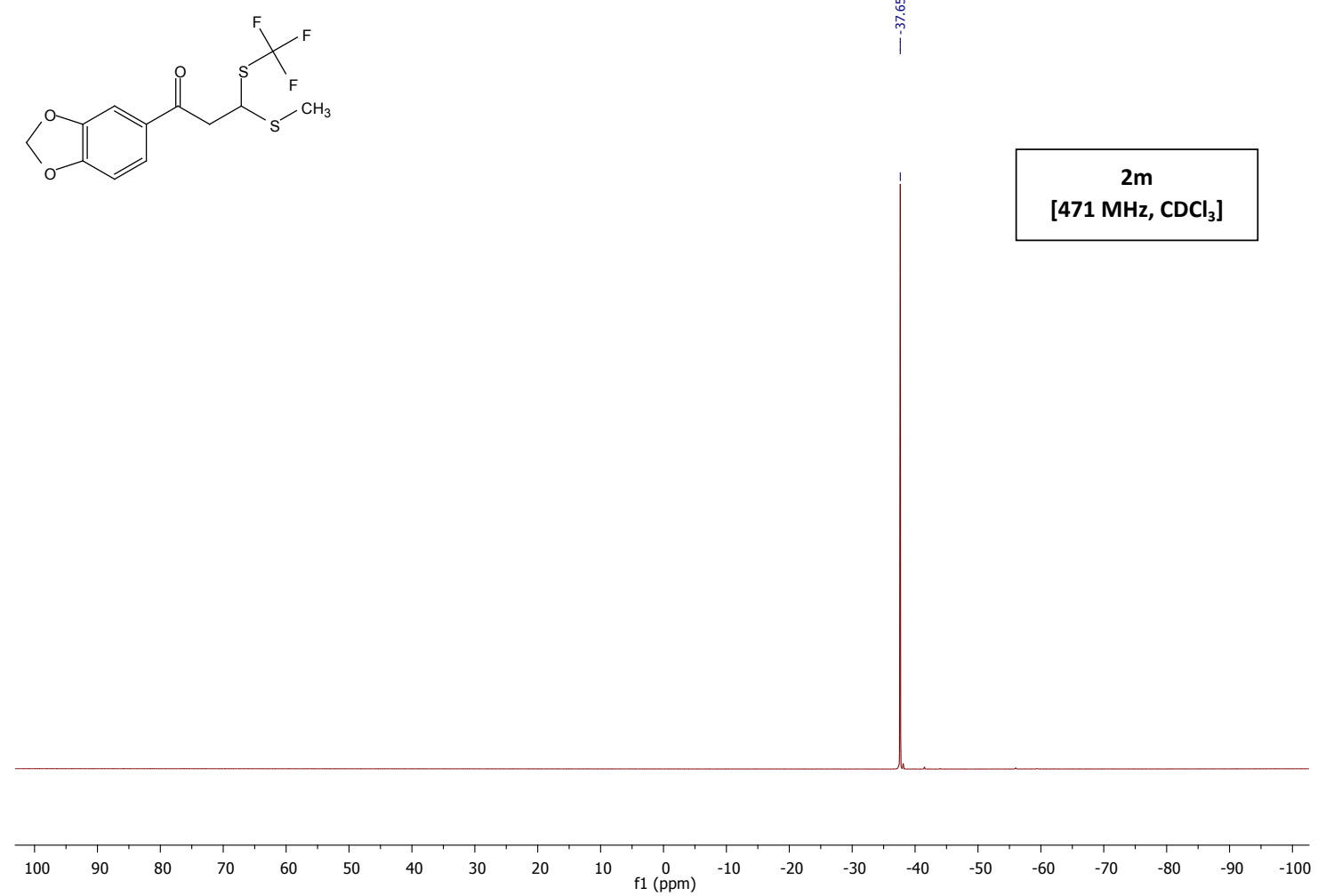







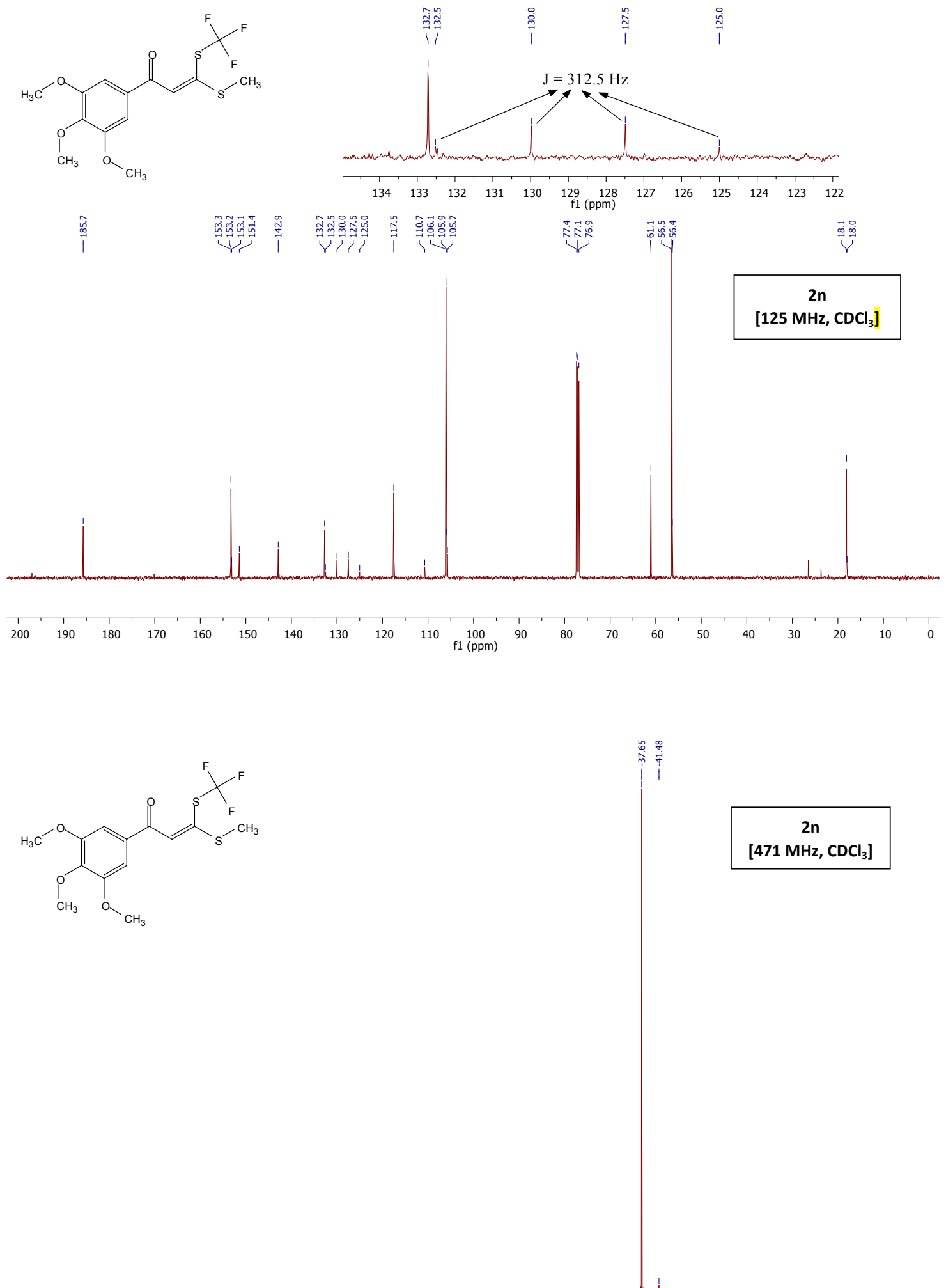

2n

[471 $\mathrm{MHz}, \mathrm{CDCl}_{3}$ ]

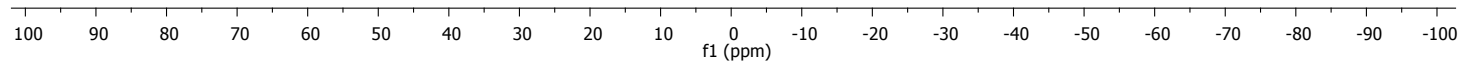


$\underline{{ }^{1} \mathrm{H} N M R,{ }^{13} \mathrm{C}\left\{{ }^{1} \mathrm{H}\right\} \text { NMR and }{ }^{19} \mathrm{~F} \text { NMR Spectra of Compound } \mathbf{2 0}}$
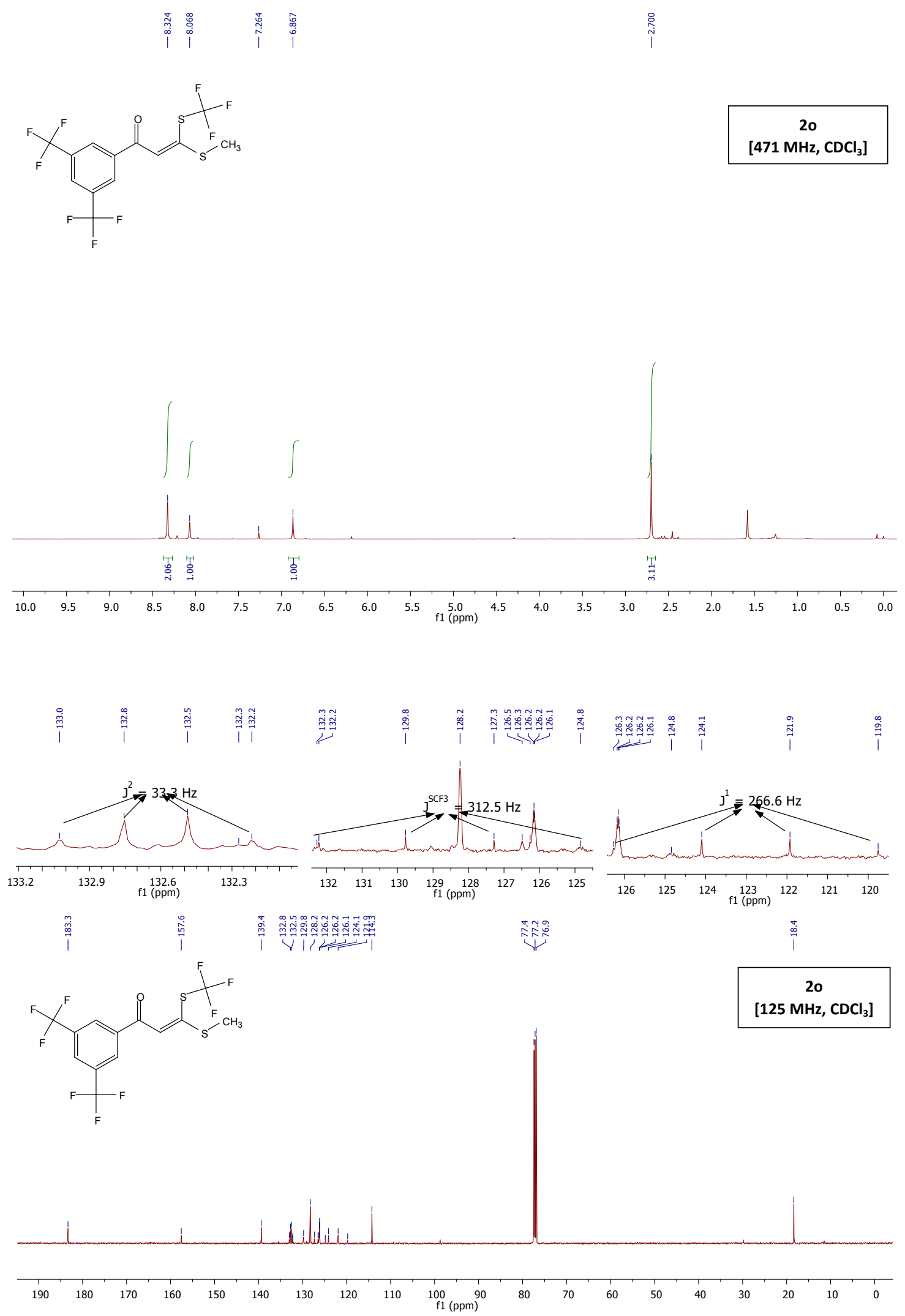

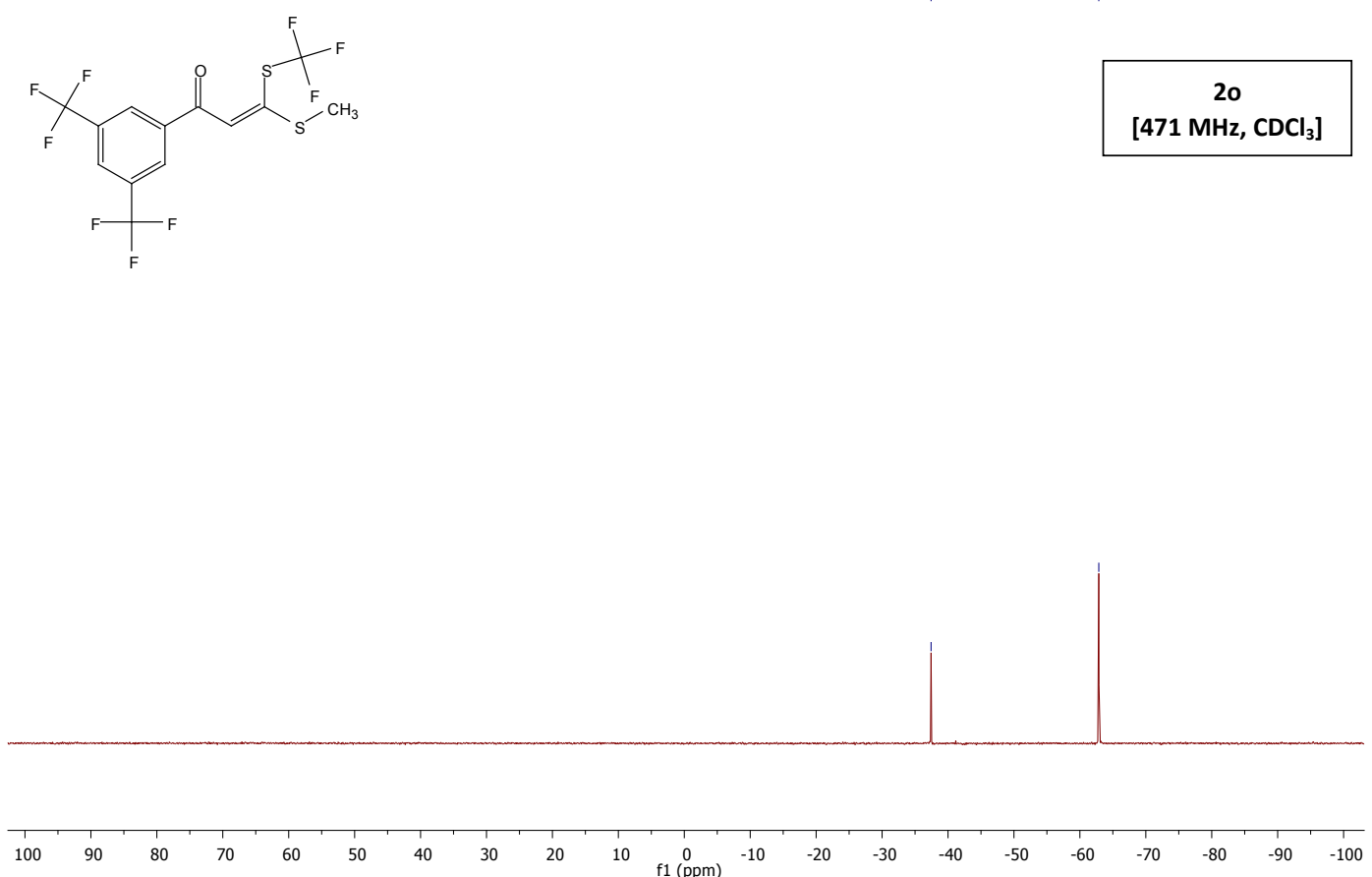

\section{$\underline{{ }^{1} \mathrm{H} N M R,{ }^{13} \mathrm{C}\left\{{ }^{1} \mathrm{H}\right\} \text { NMR and }{ }^{19} \mathrm{~F} \text { NMR Spectra of Compound } \mathbf{2 p}}$}
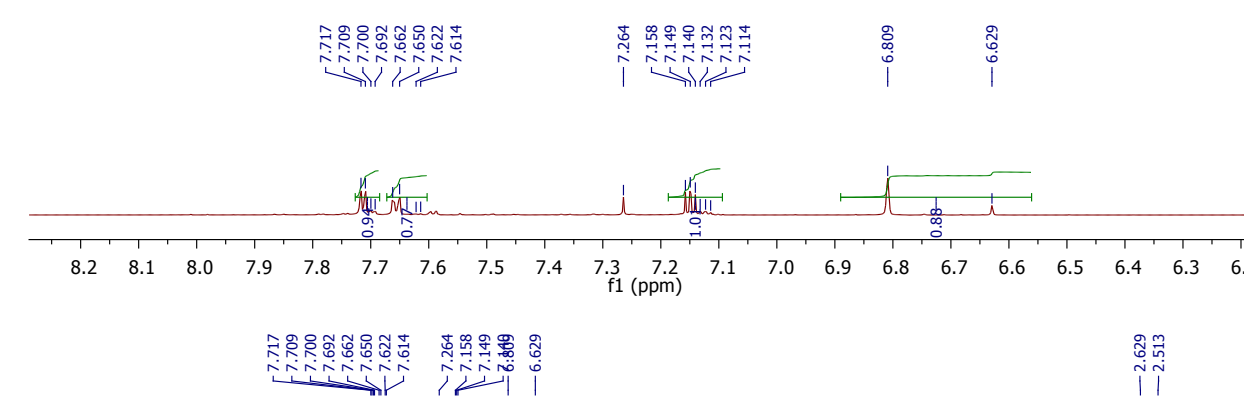

ํํำ

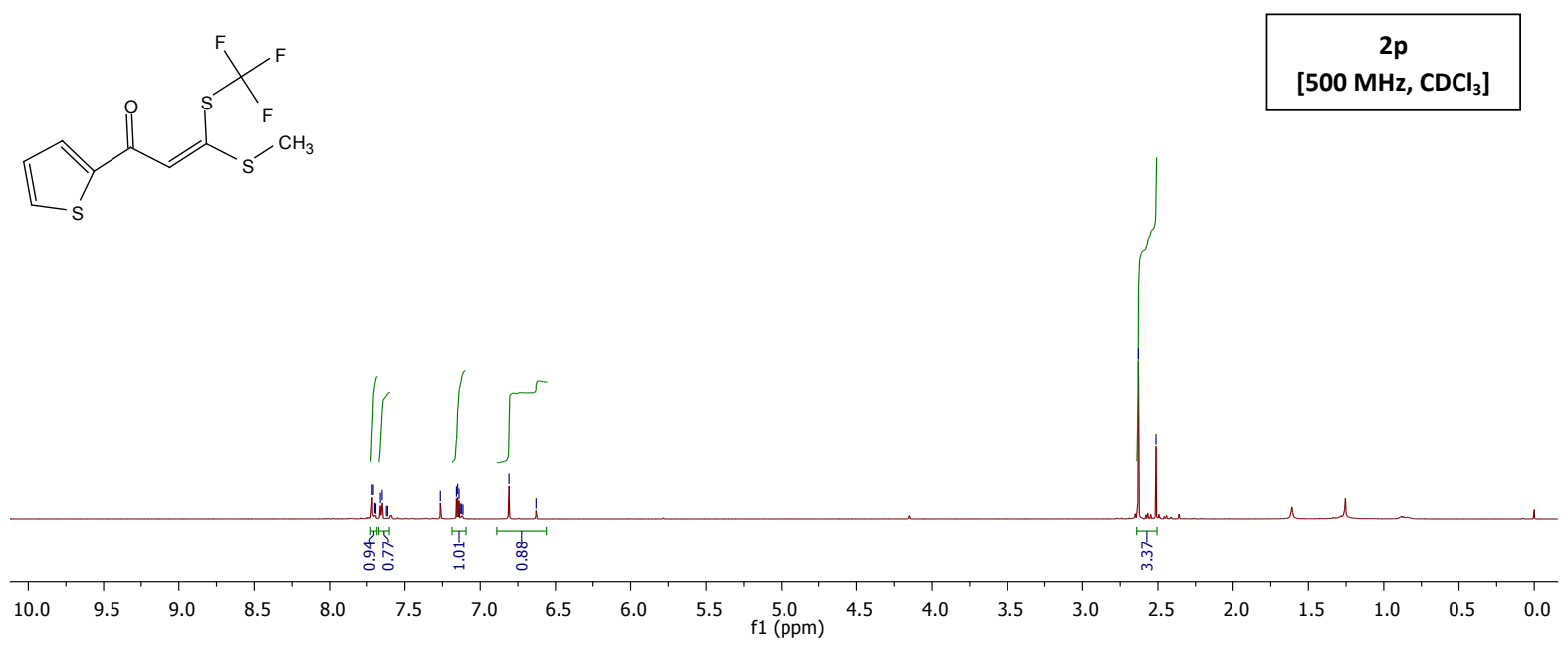



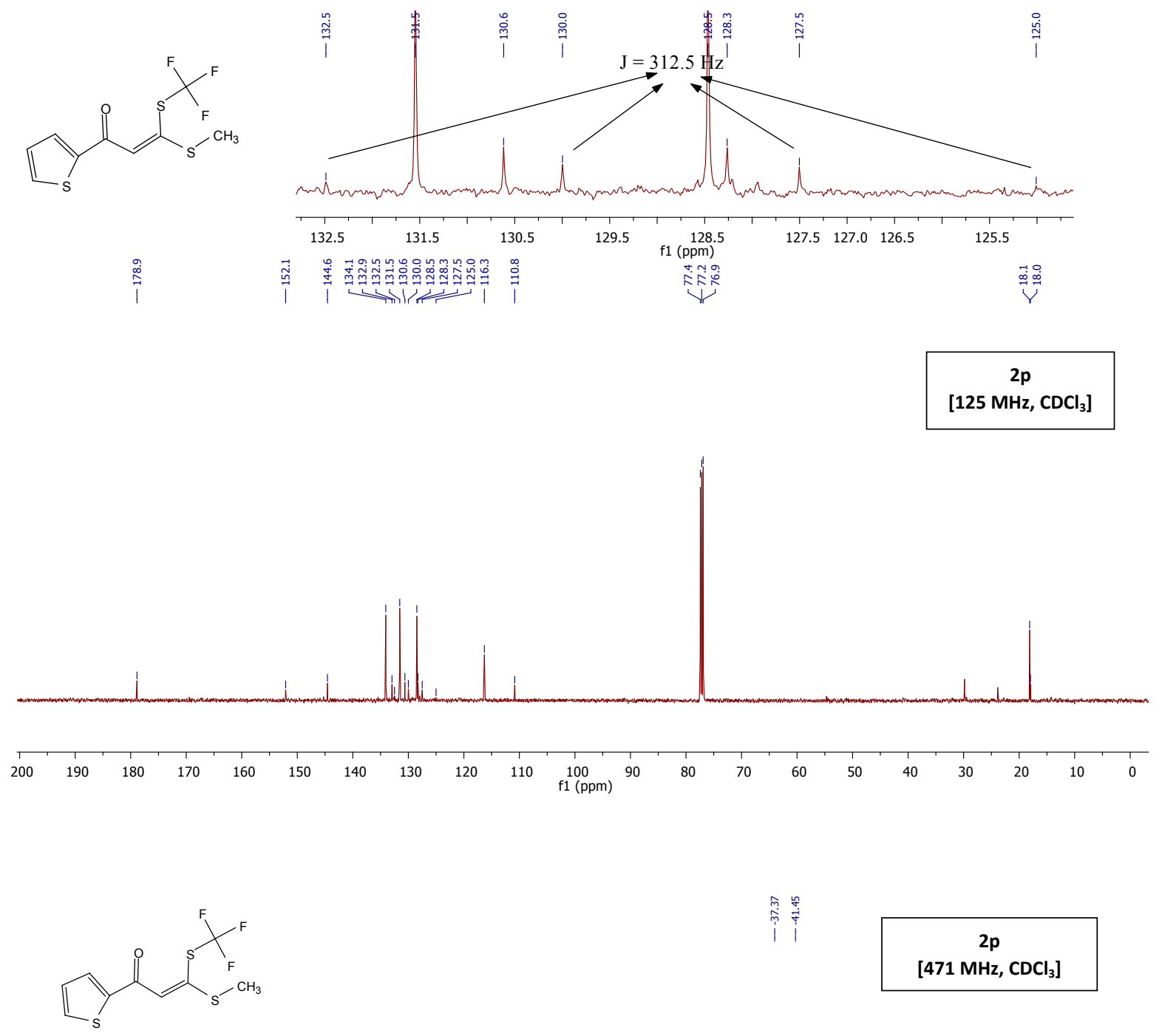

if

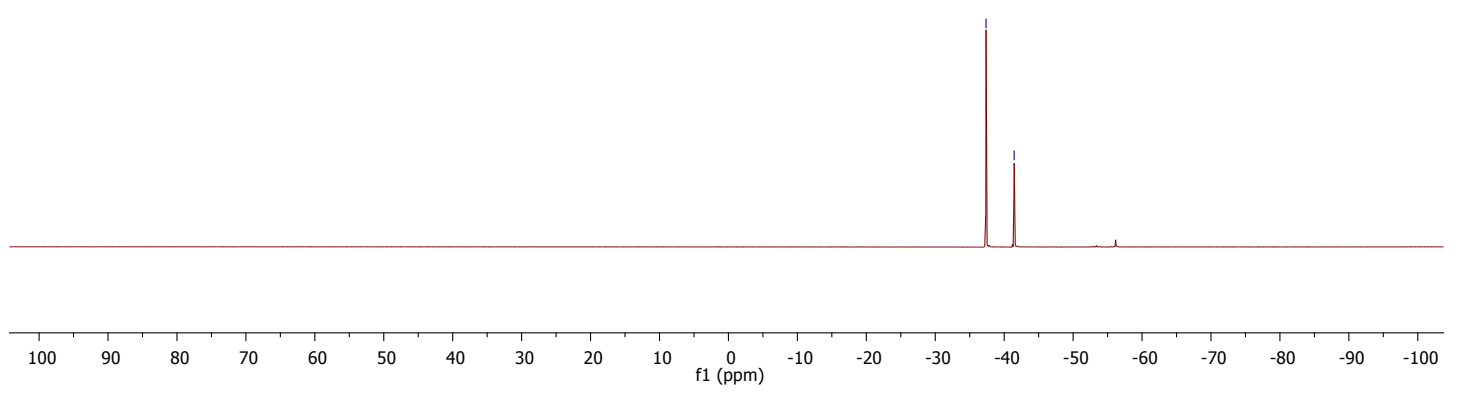


$\underline{{ }^{1} \mathrm{H} N M R,{ }^{13} \mathrm{C}\left\{{ }^{1} \mathrm{H}\right\} \text { NMR and }{ }^{19} \mathrm{~F} \text { NMR Spectra of Compound } \mathbf{2 q}}$

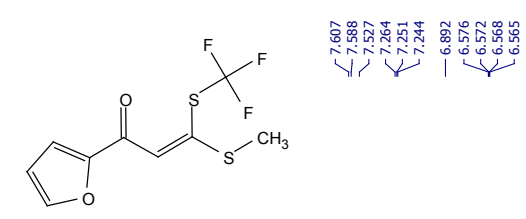

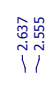


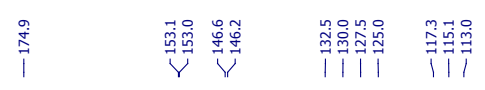
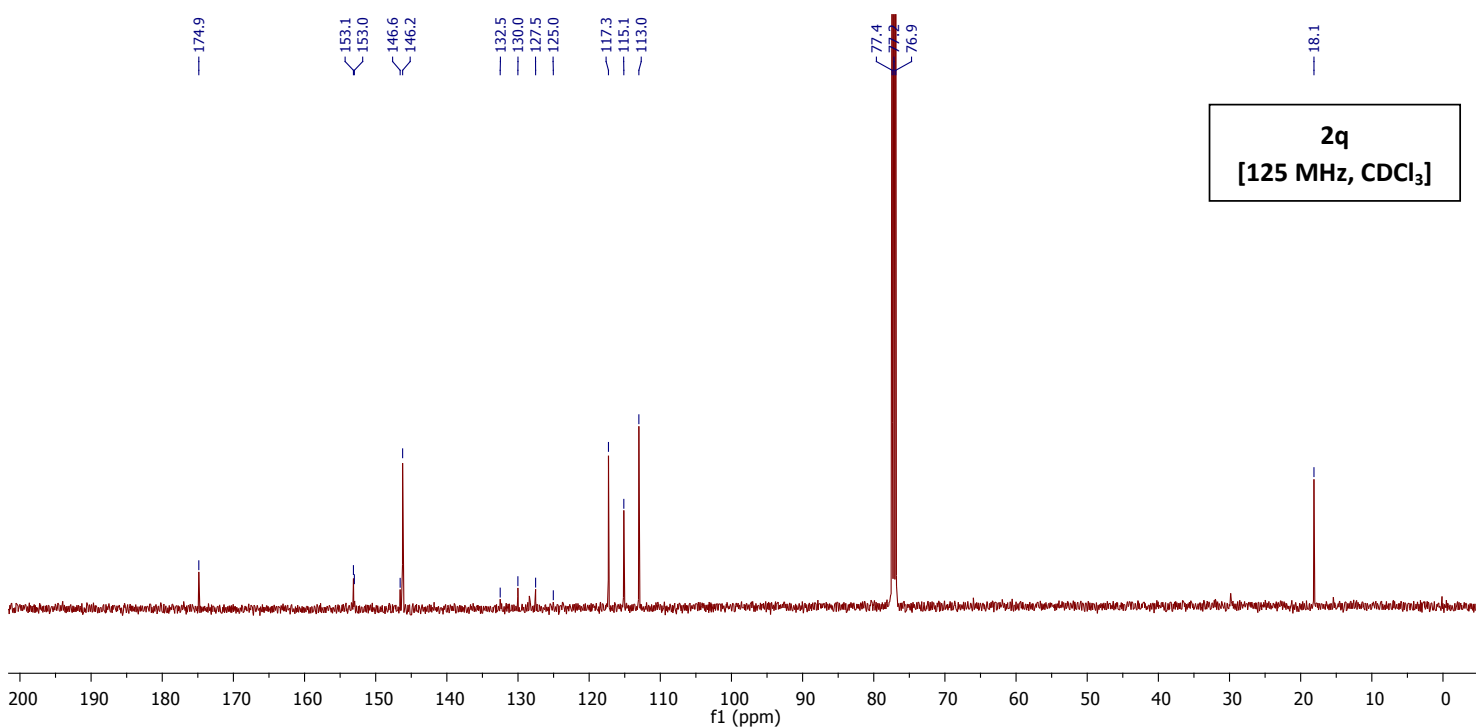


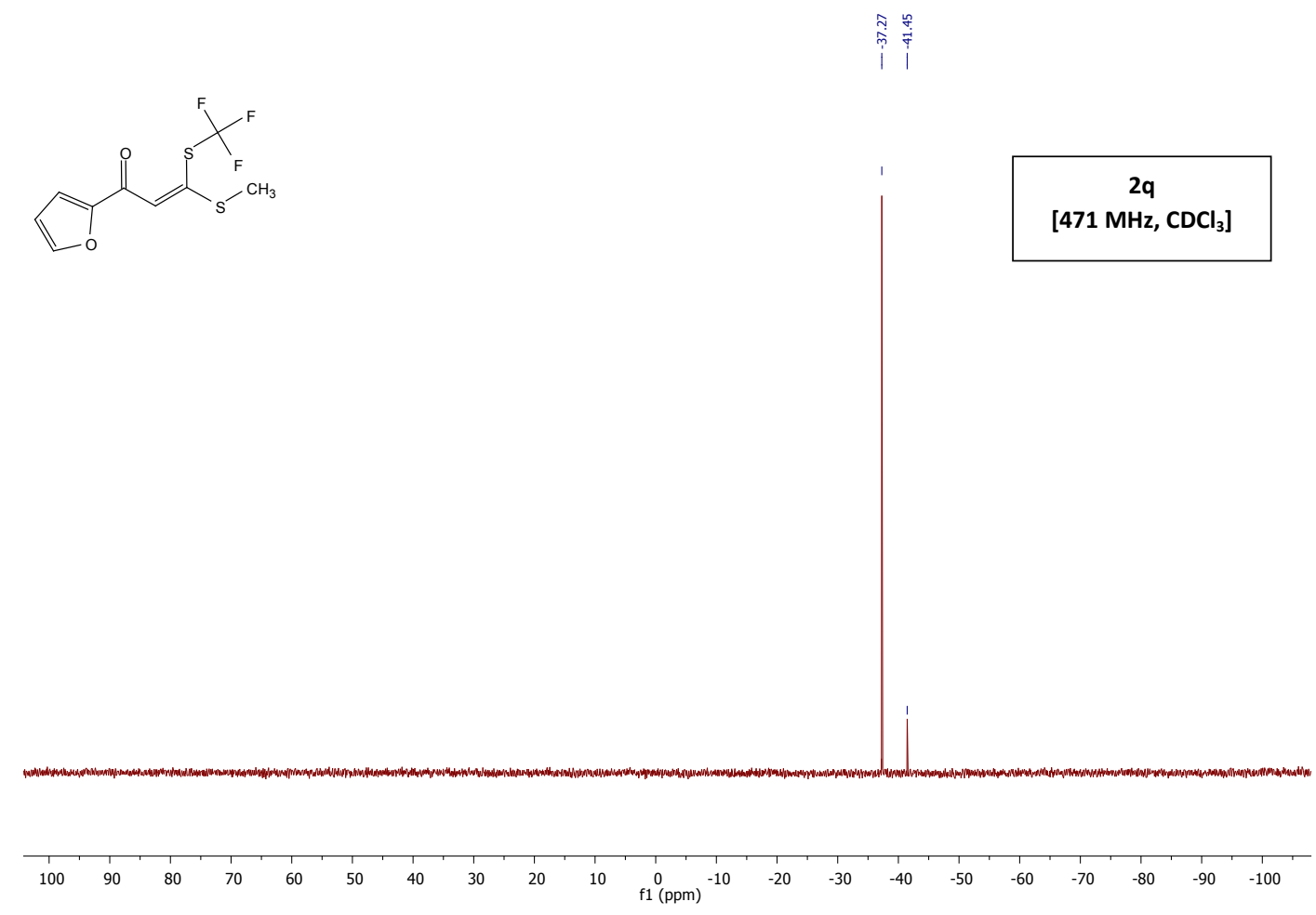

$\underline{{ }^{1} \mathrm{H} N M R,{ }^{13} \mathrm{C}\left\{{ }^{1} \mathrm{H}\right\} \text { NMR and }{ }^{19} \mathrm{~F} \text { NMR Spectra of Compound } \mathbf{2 r}}$

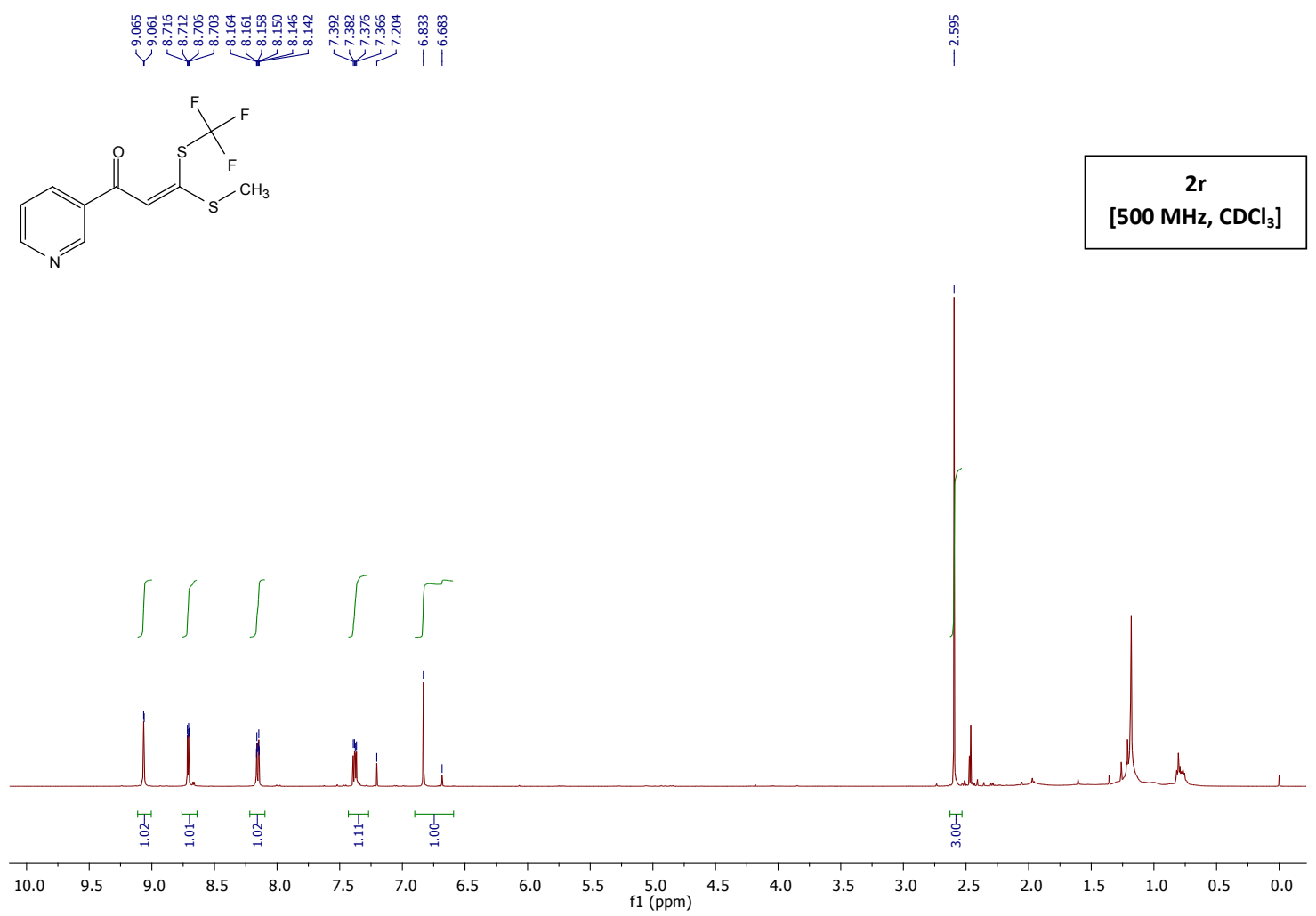



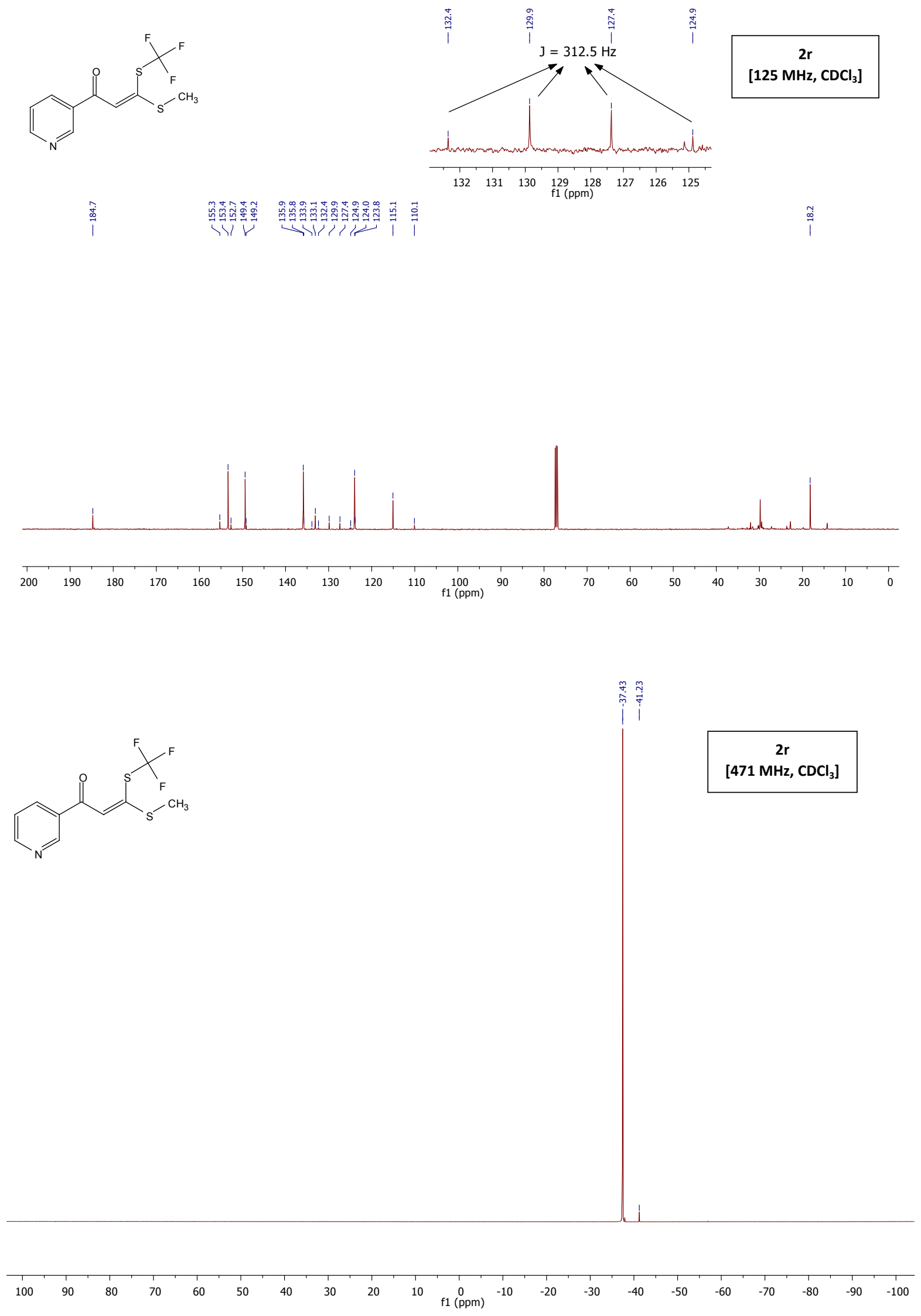
$\underline{{ }^{1} \mathrm{H} \text { NMR, }{ }^{13} \mathrm{C}\left\{{ }^{1} \mathrm{H}\right\} \text { NMR and }{ }^{19} \mathrm{~F} \text { NMR Spectra of Compound } \mathbf{2 s}}$
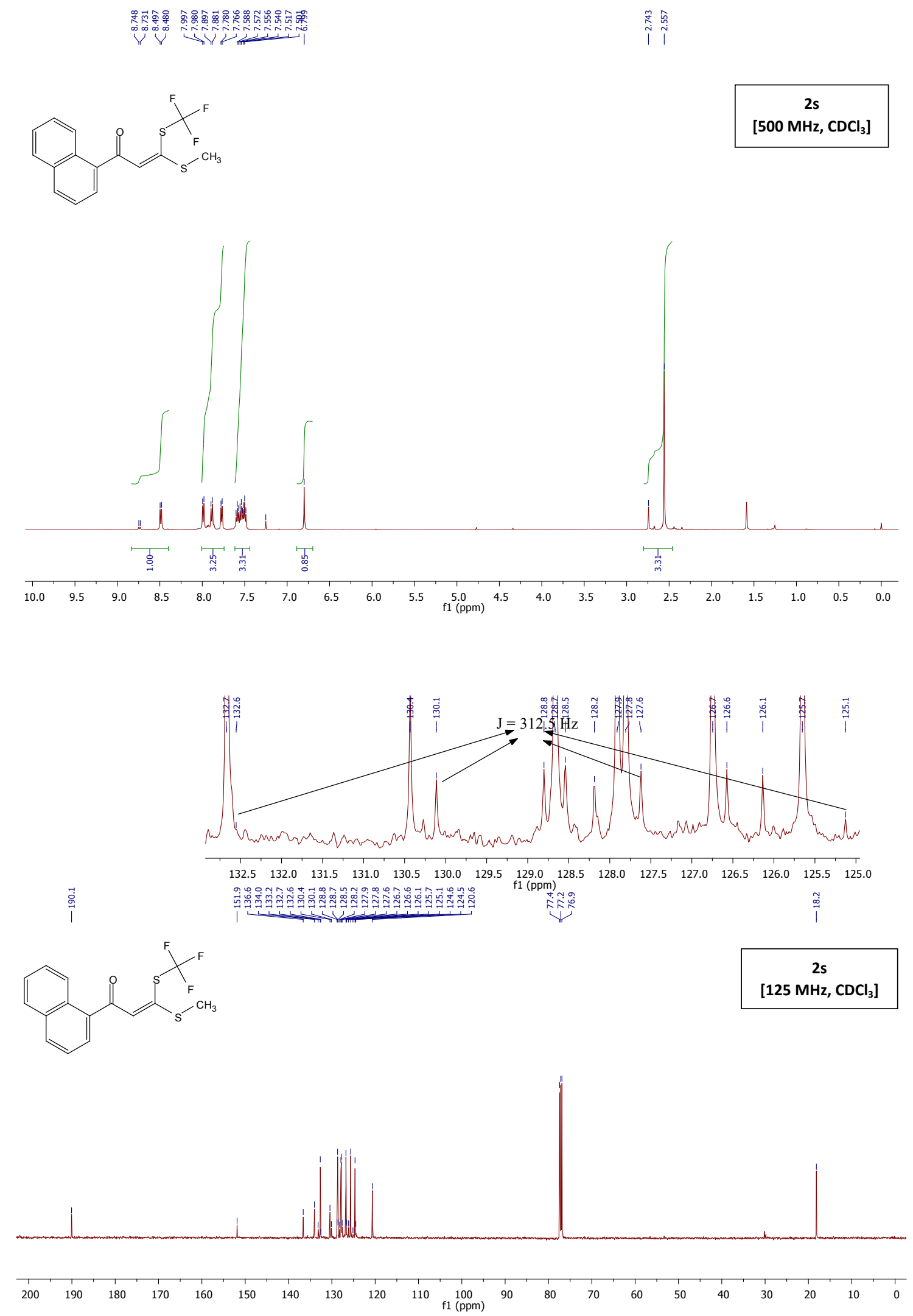


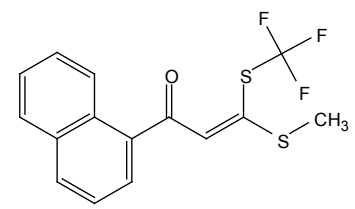

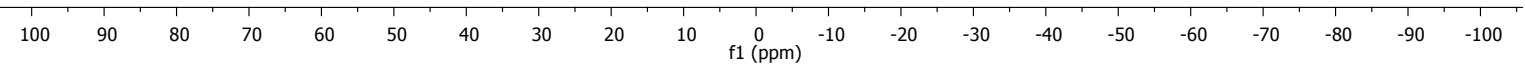

\section{$\underline{{ }^{1} \mathrm{H} N M R,{ }^{13} \mathrm{C}\left\{{ }^{1} \mathrm{H}\right\} \text { NMR and }{ }^{19} \mathrm{~F} \text { NMR Spectra of Compound } \mathbf{2 t}}$}

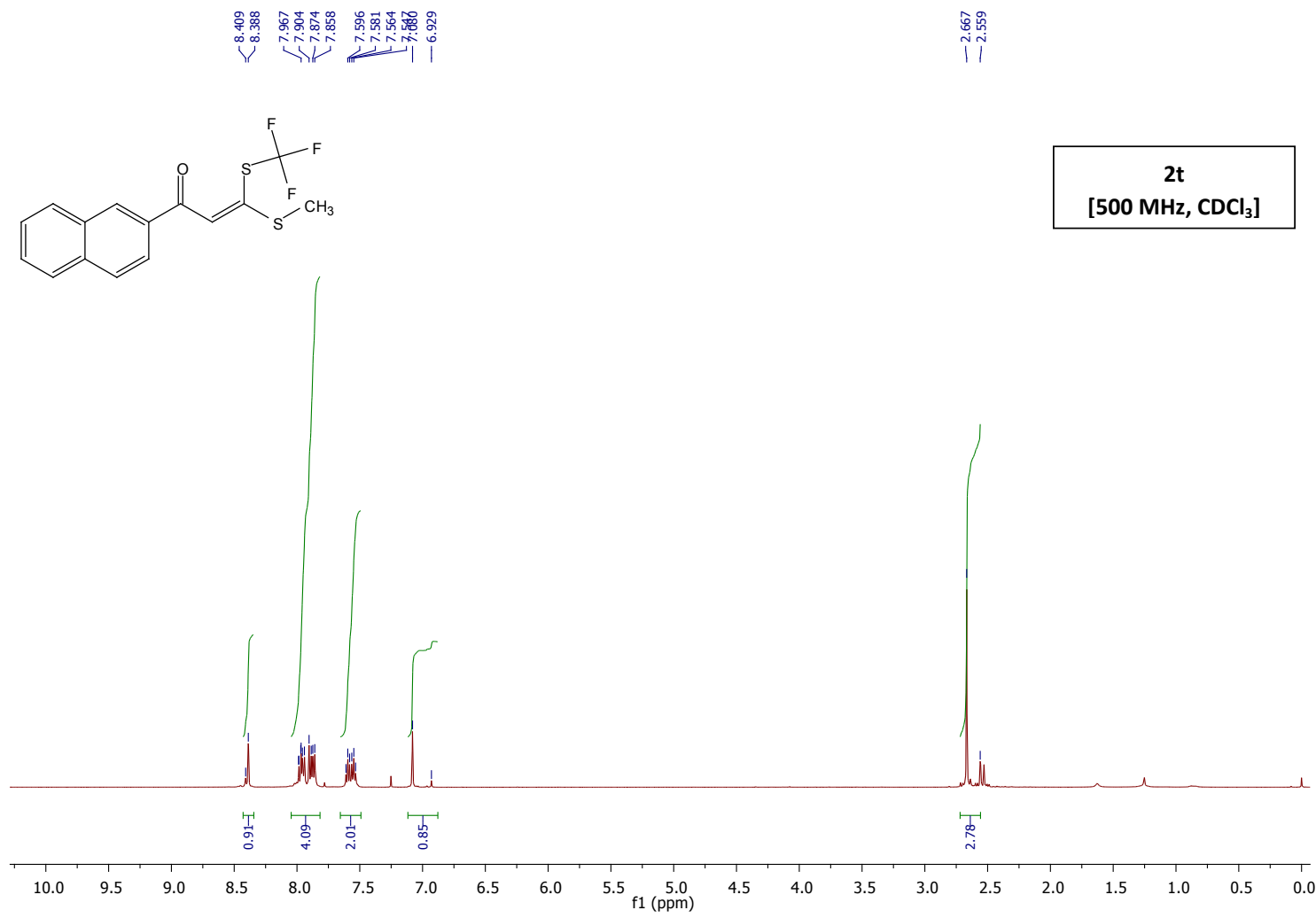




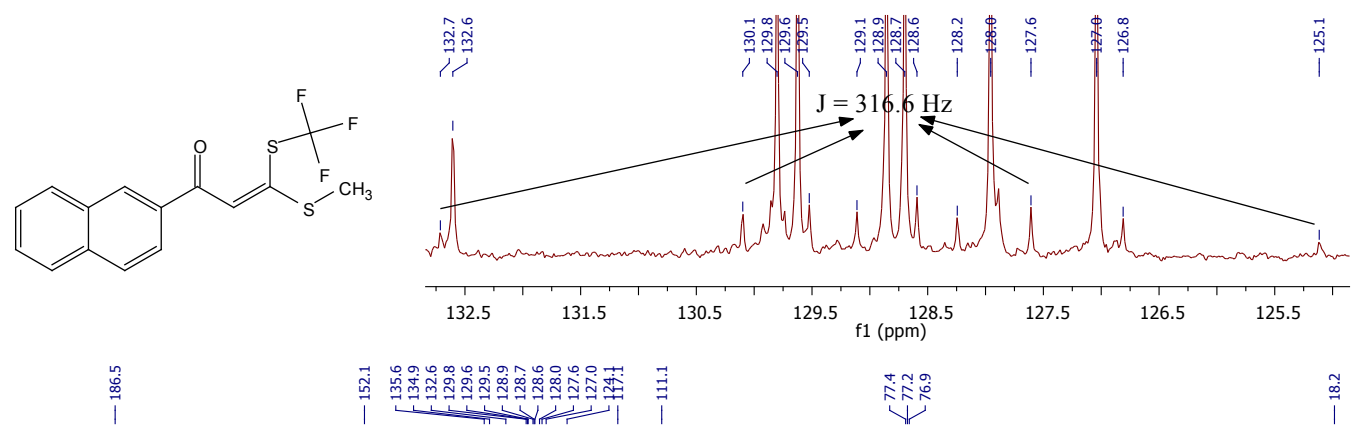

$2 \mathrm{t}$

[125 $\mathrm{MHz} \mathrm{CDCl}_{3}$ ]

$\underline{{ }^{1} \mathrm{H} N M R,{ }^{13} \mathrm{C}\left\{{ }^{1} \mathrm{H}\right\} \text { NMR and }{ }^{19} \mathrm{~F} \text { NMR Spectra of Compound } \mathbf{2 u}}$


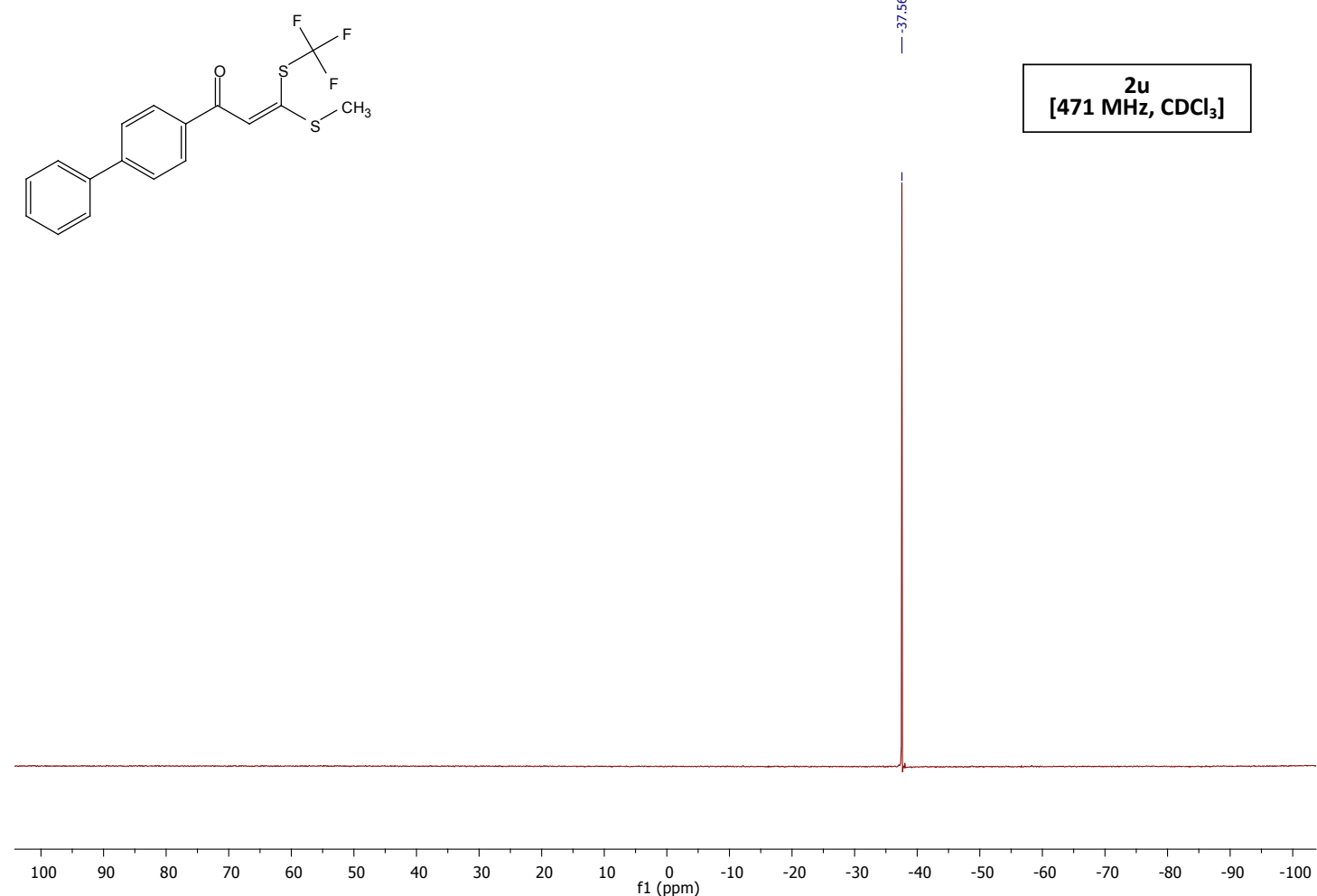

$\underline{{ }^{1} \mathrm{H} N M R,{ }^{13} \mathrm{C}\left\{{ }^{1} \mathrm{H}\right\} \text { NMR and }{ }^{19} \mathrm{~F} \text { NMR Spectra of Compound } \mathbf{2 v}}$

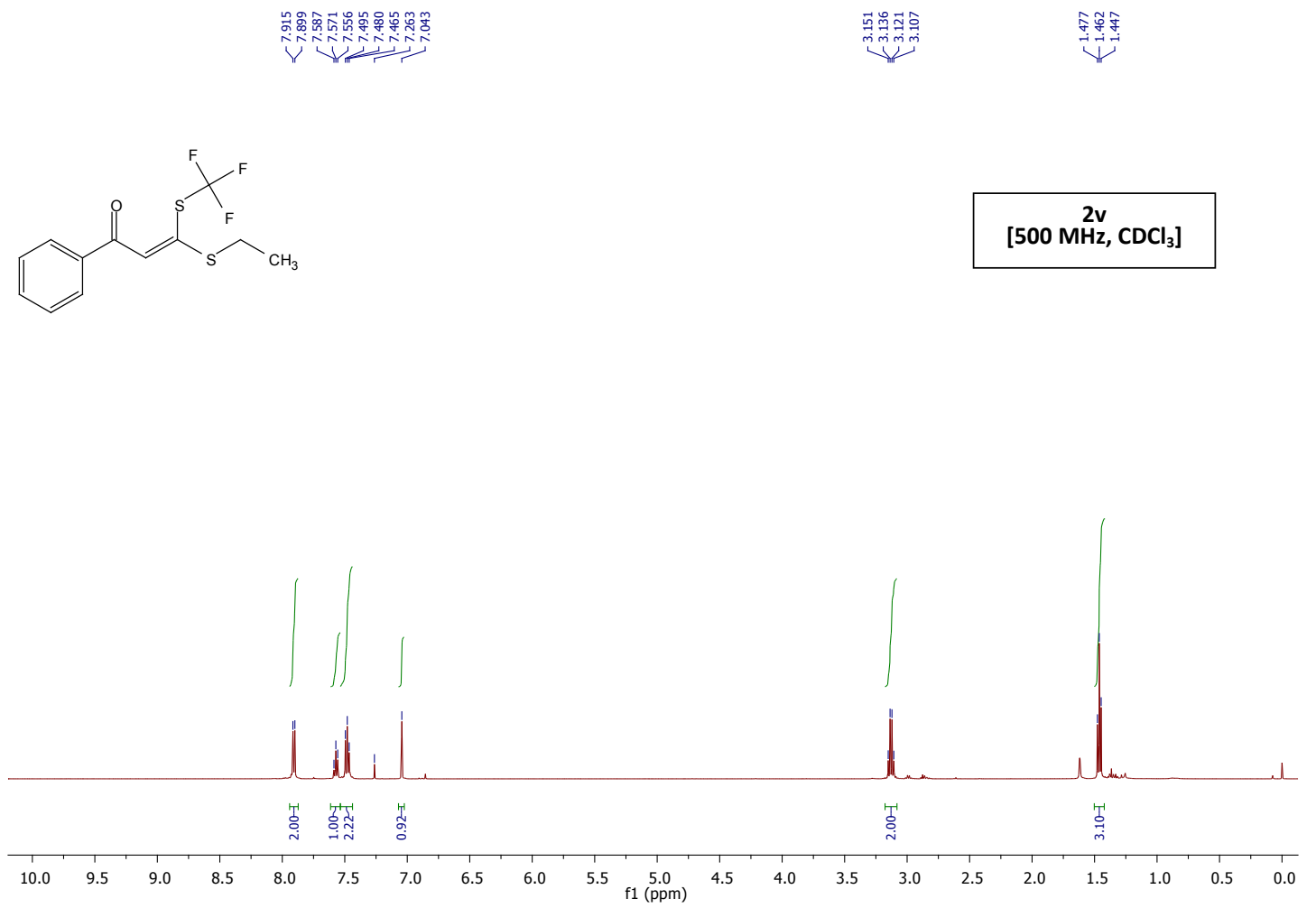



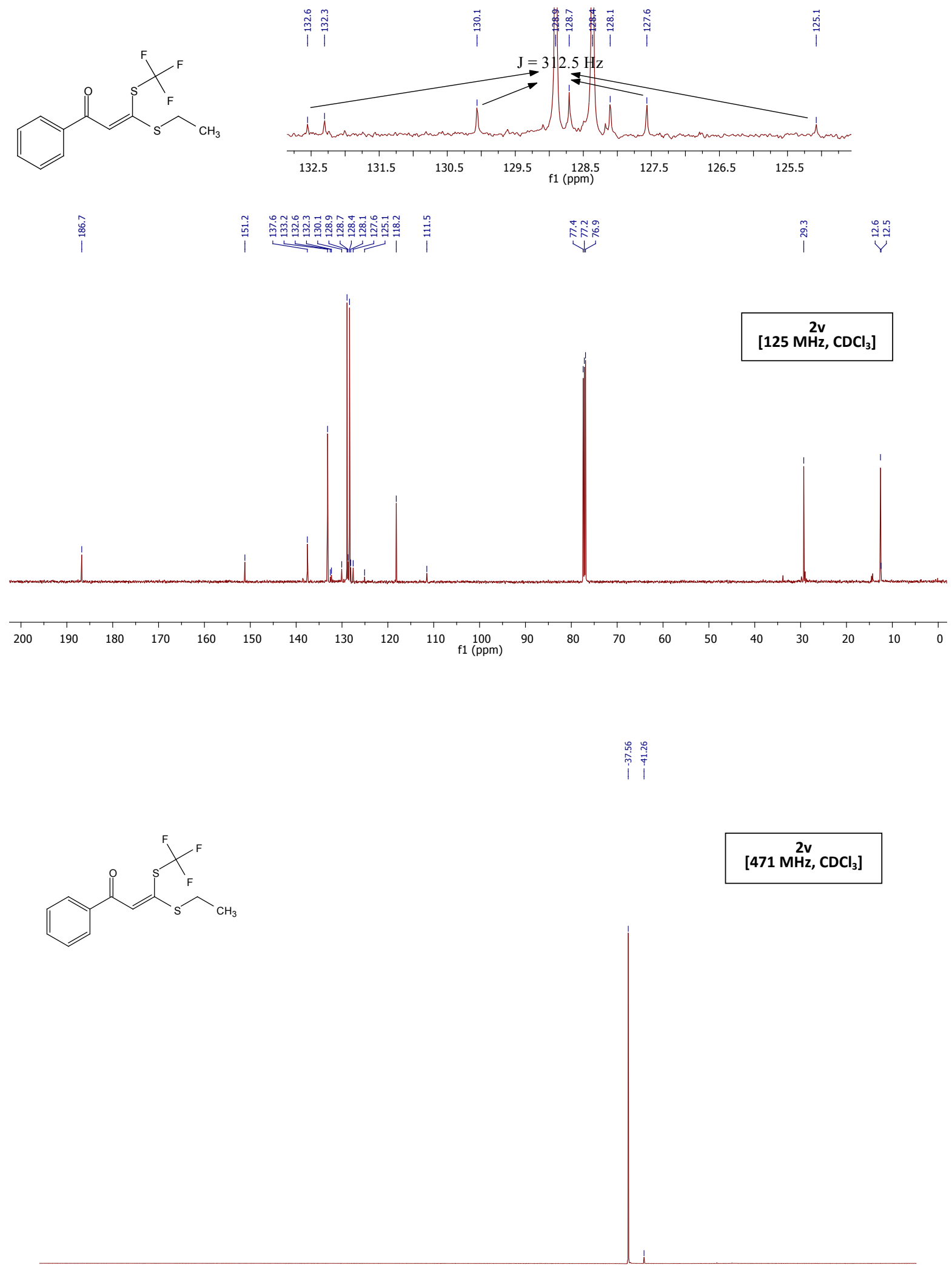




$\underline{{ }^{1} \mathrm{H} N M R,{ }^{13} \mathrm{C}\left\{{ }^{1} \mathrm{H}\right\} \text { NMR and }{ }^{19} \mathrm{~F} \text { NMR Spectra of Compound } \mathbf{2 w}}$
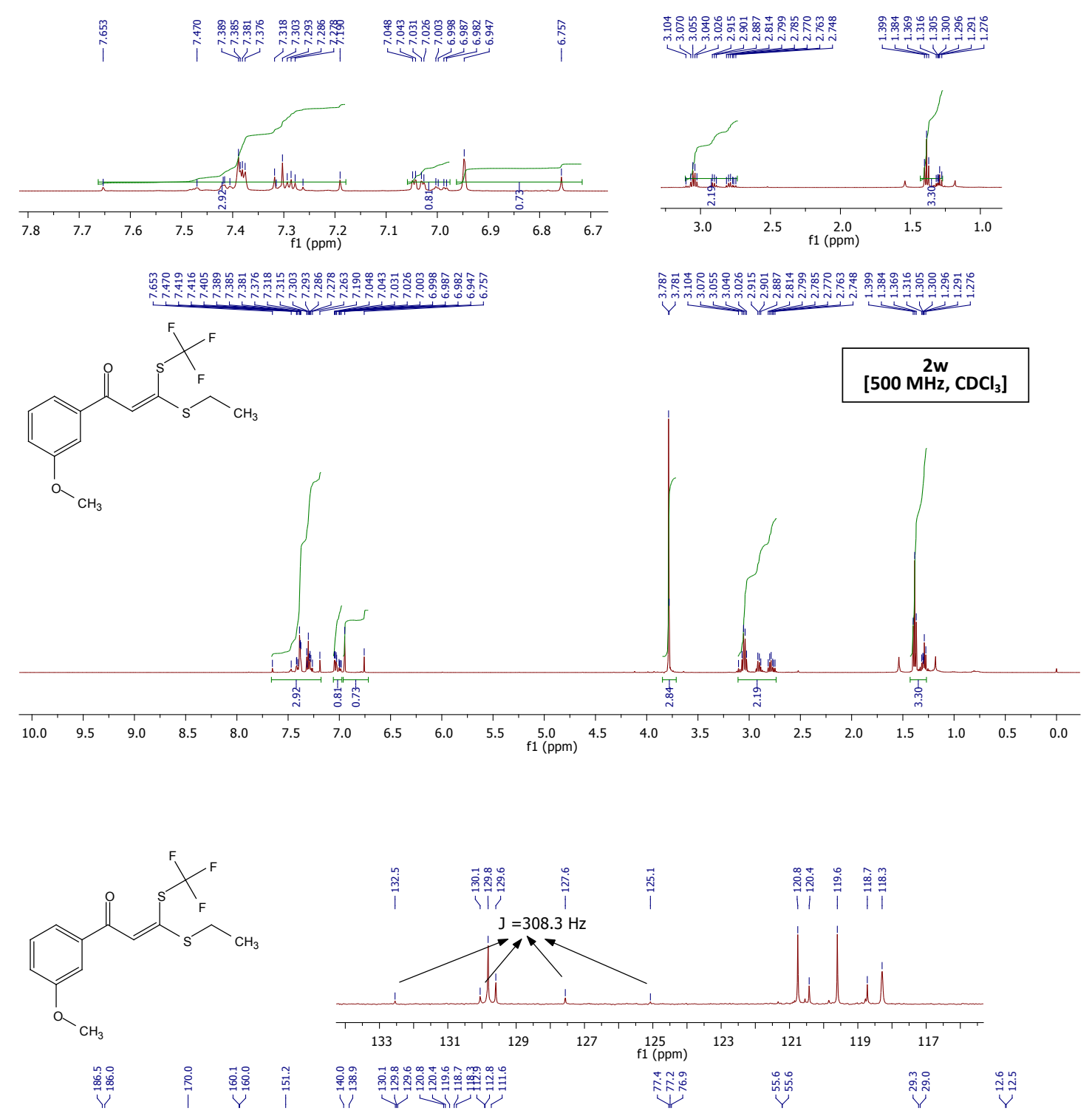

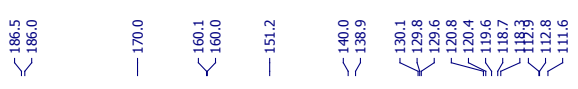

[125 MHz, $\left.\mathrm{CDCl}_{3}\right]$

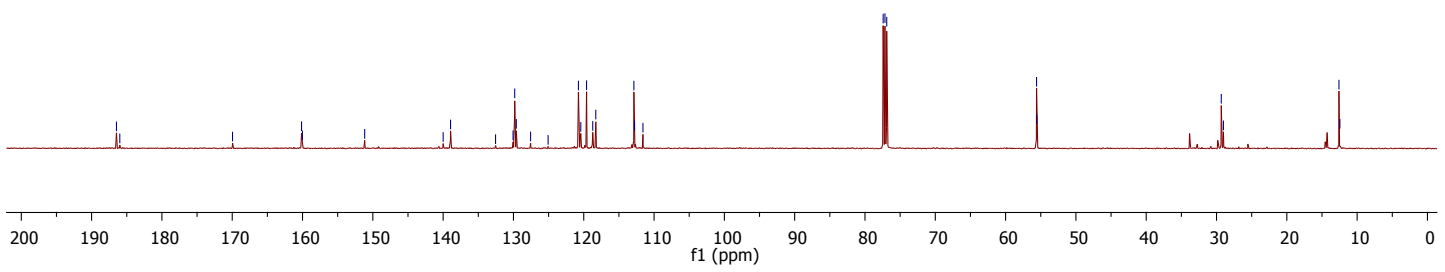






 [471 $\left.\mathrm{MHz}^{2 \mathrm{w}} \mathrm{CDCl}_{3}\right]$

\begin{tabular}{rlllllllllllllllllllllll}
\hline & 100 & 90 & 80 & 70 & 60 & 50 & 40 & 30 & 20 & 10 & $\underset{\mathrm{f} 1(\mathrm{ppm})}{0}$ & -10 & -20 & -30 & -40 & -50 & -60 & -70 & -80 & -90 & -100
\end{tabular}

$\underline{{ }^{1} \mathrm{H} N M R,{ }^{13} \mathrm{C}\left\{{ }^{1} \mathrm{H}\right\} \text { NMR and }{ }^{19} \mathrm{~F} \text { NMR Spectra of Compound } \mathbf{2 x}}$
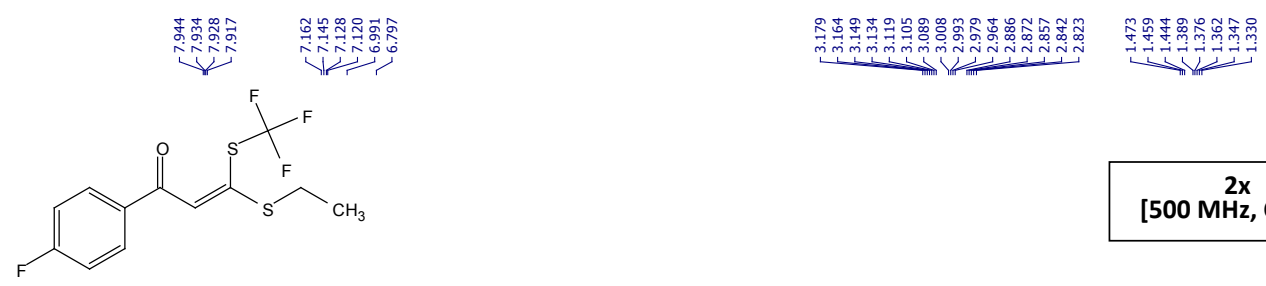

$\left[500 \mathrm{MHz}^{2 \mathrm{x}} \mathrm{CDCl}_{3}\right]$

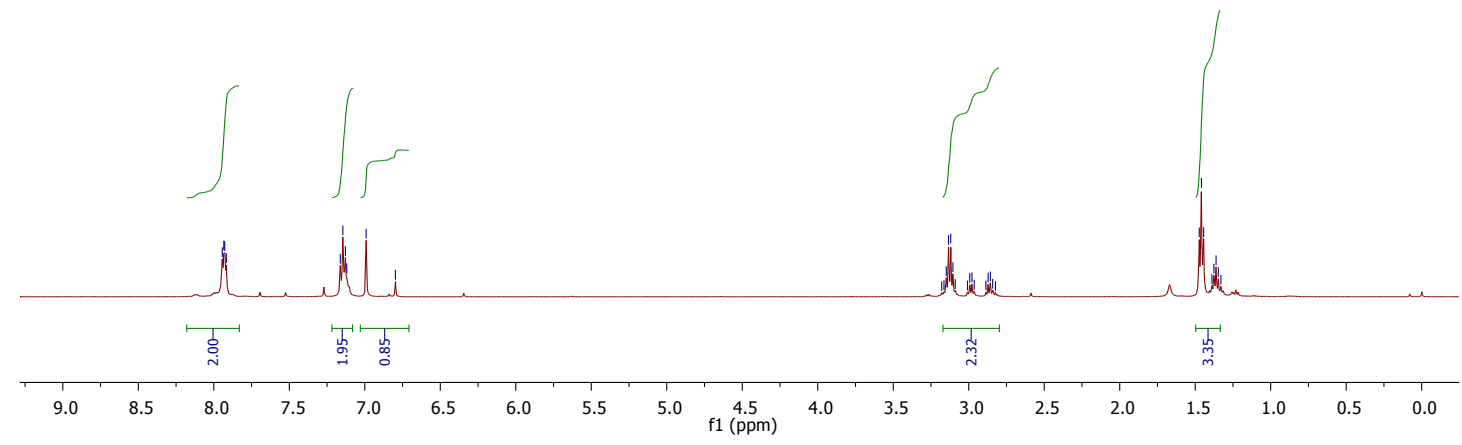





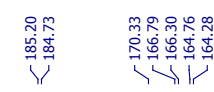



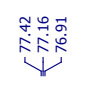

ปู<smiles>CCS/C(=C/C(=O)c1ccc(F)cc1)SC(F)(F)F</smiles>

\section{$\left[500 \mathrm{MHz}^{2 \mathrm{x}}, \mathrm{CDCl}_{3}\right]$}


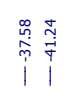

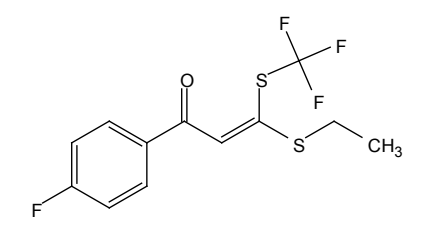

$\left[471 \mathrm{MHz}, \mathrm{CDCl}_{3}\right]$

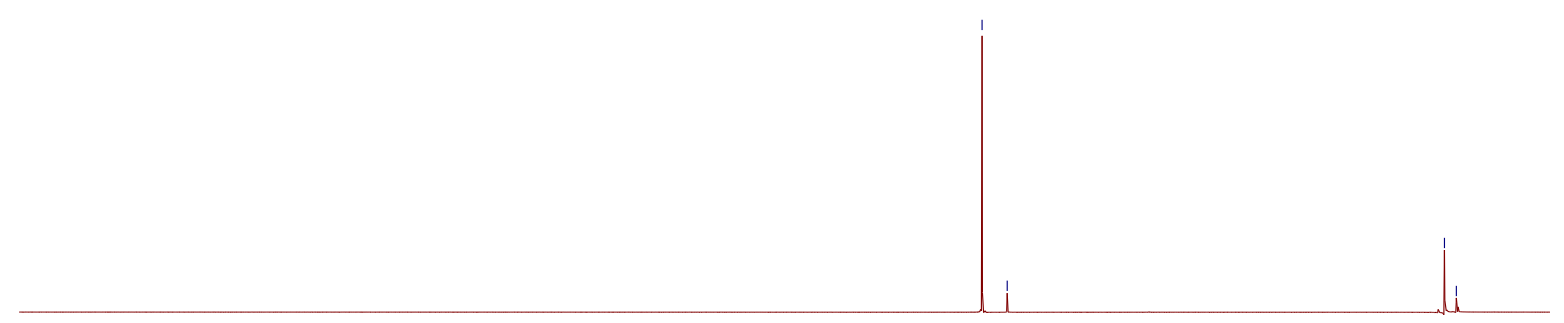

$\begin{array}{llllllllllllllllllllllllll}1 & 100 & 90 & 80 & 70 & 60 & 50 & 40 & 30 & 20 & 10 & 0 & -10 & -20 & -30 & -40 & -50 & -60 & -70 & -80 & -90 & -100 & -110 & -126\end{array}$ 
$\underline{{ }^{1} \mathrm{H} N M R,{ }^{13} \mathrm{C}\left\{{ }^{1} \mathrm{H}\right\} \text { NMR and }{ }^{19} \mathrm{~F} \text { NMR Spectra of Compound } \mathbf{2 y}}$
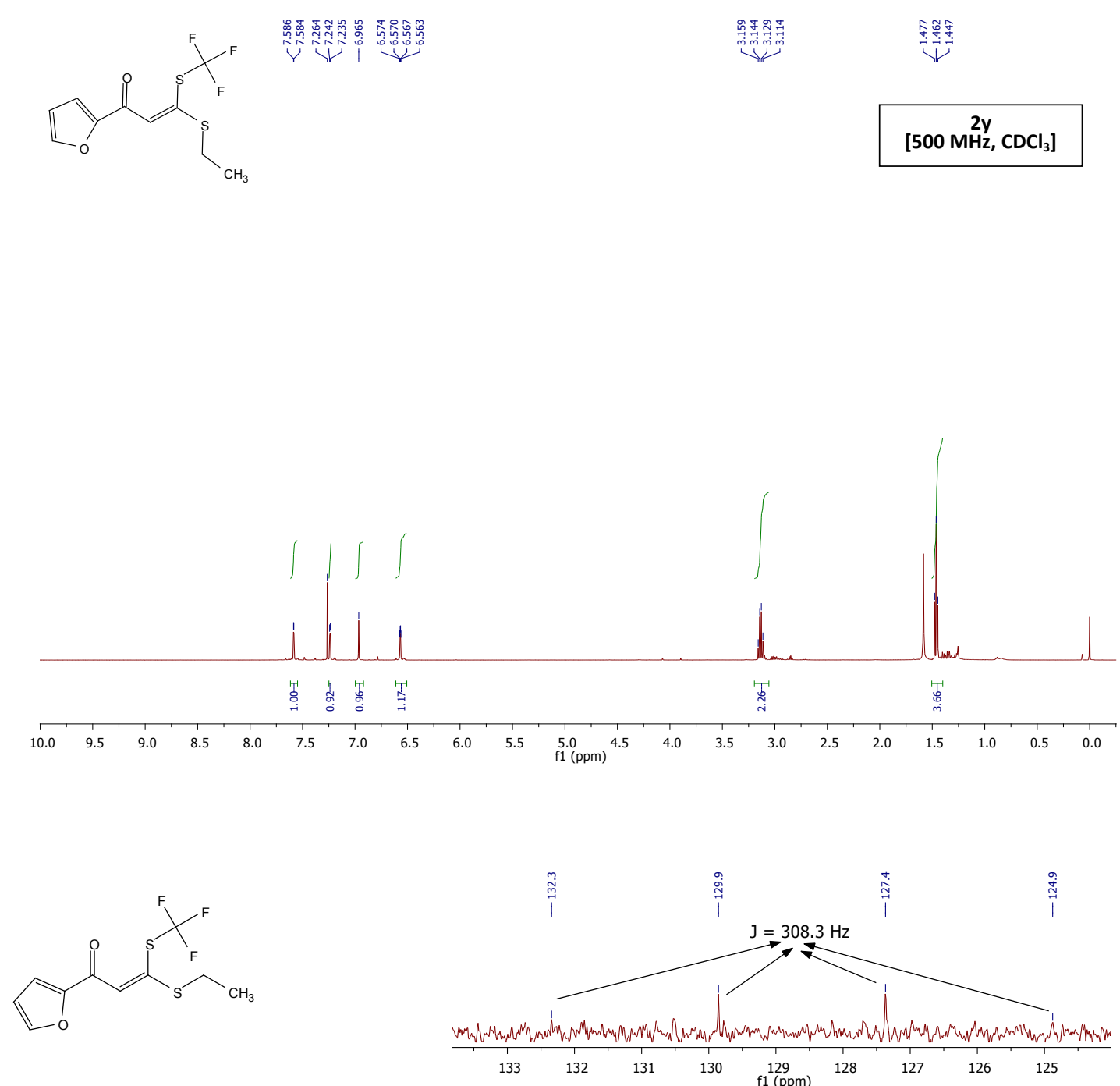



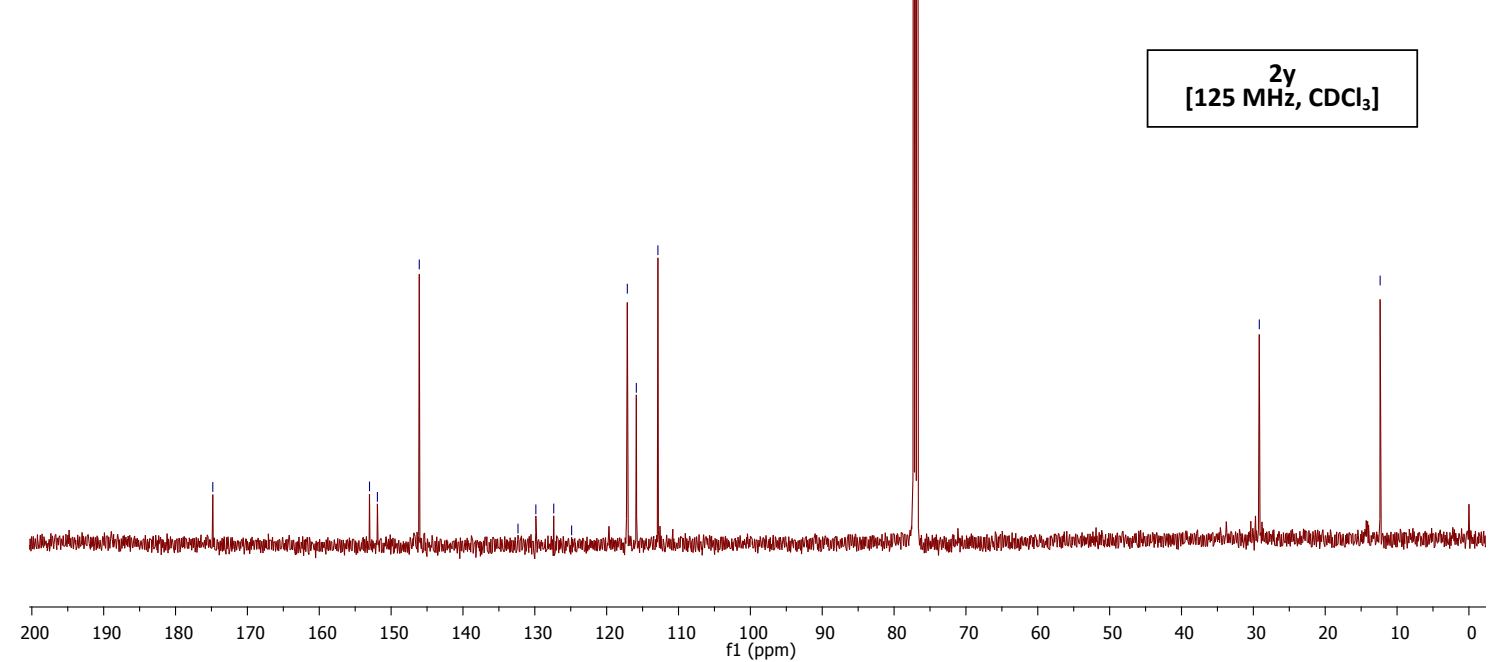




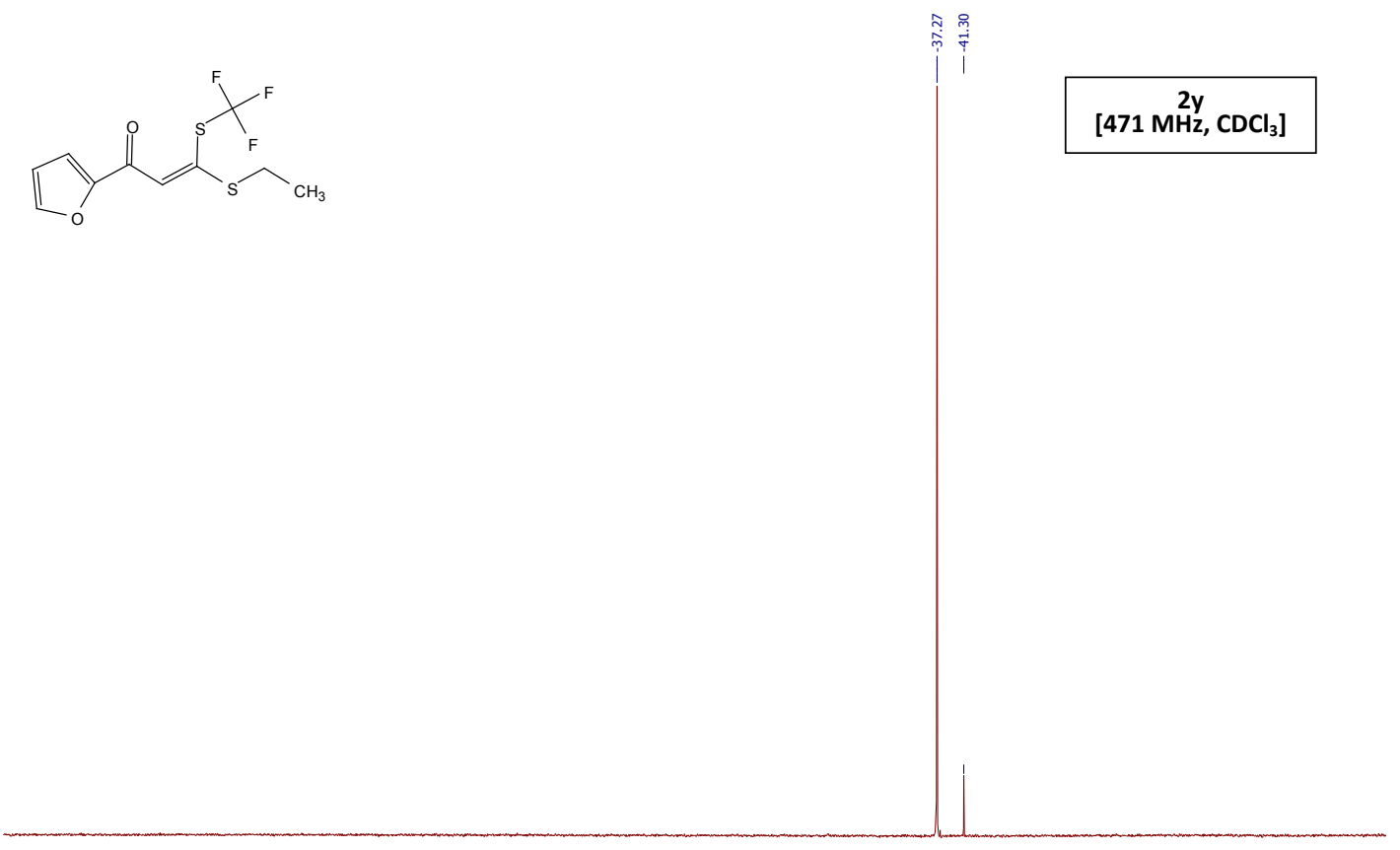

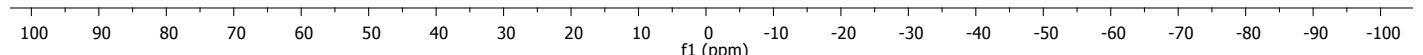

$\underline{{ }^{1} \mathrm{H} N M R,{ }^{13} \mathrm{C}\left\{{ }^{1} \mathrm{H}\right\} \text { NMR and }{ }^{19} \mathrm{~F} \text { NMR Spectra of Compound } \mathbf{2 z}}$


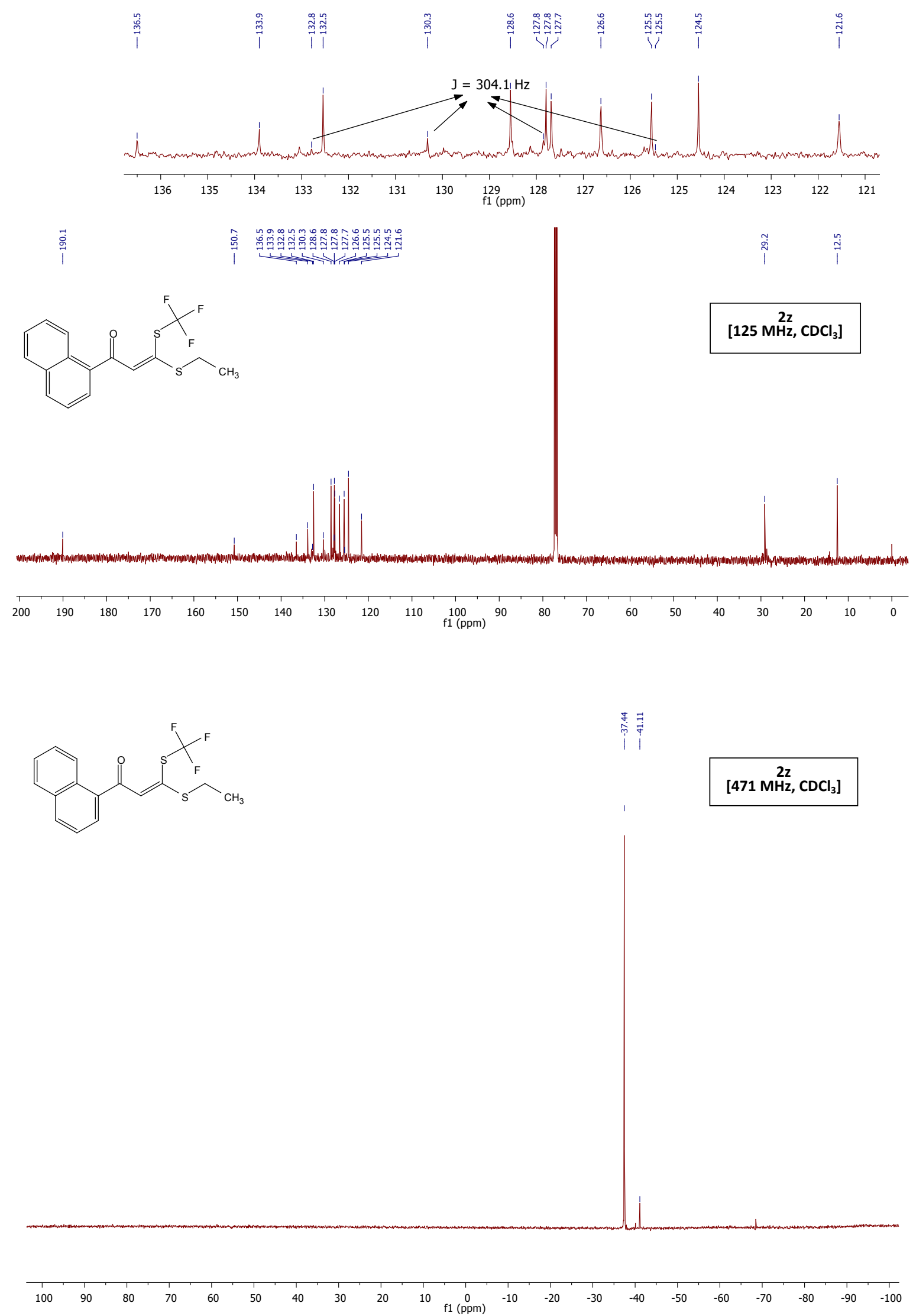
$\underline{{ }^{1} \mathrm{H} \text { NMR, }{ }^{13} \mathrm{C}\left\{{ }^{1} \mathrm{H}\right\} \text { NMR and }{ }^{19} \mathrm{~F} \text { NMR Spectra of Compound } \mathbf{2 b a}}$

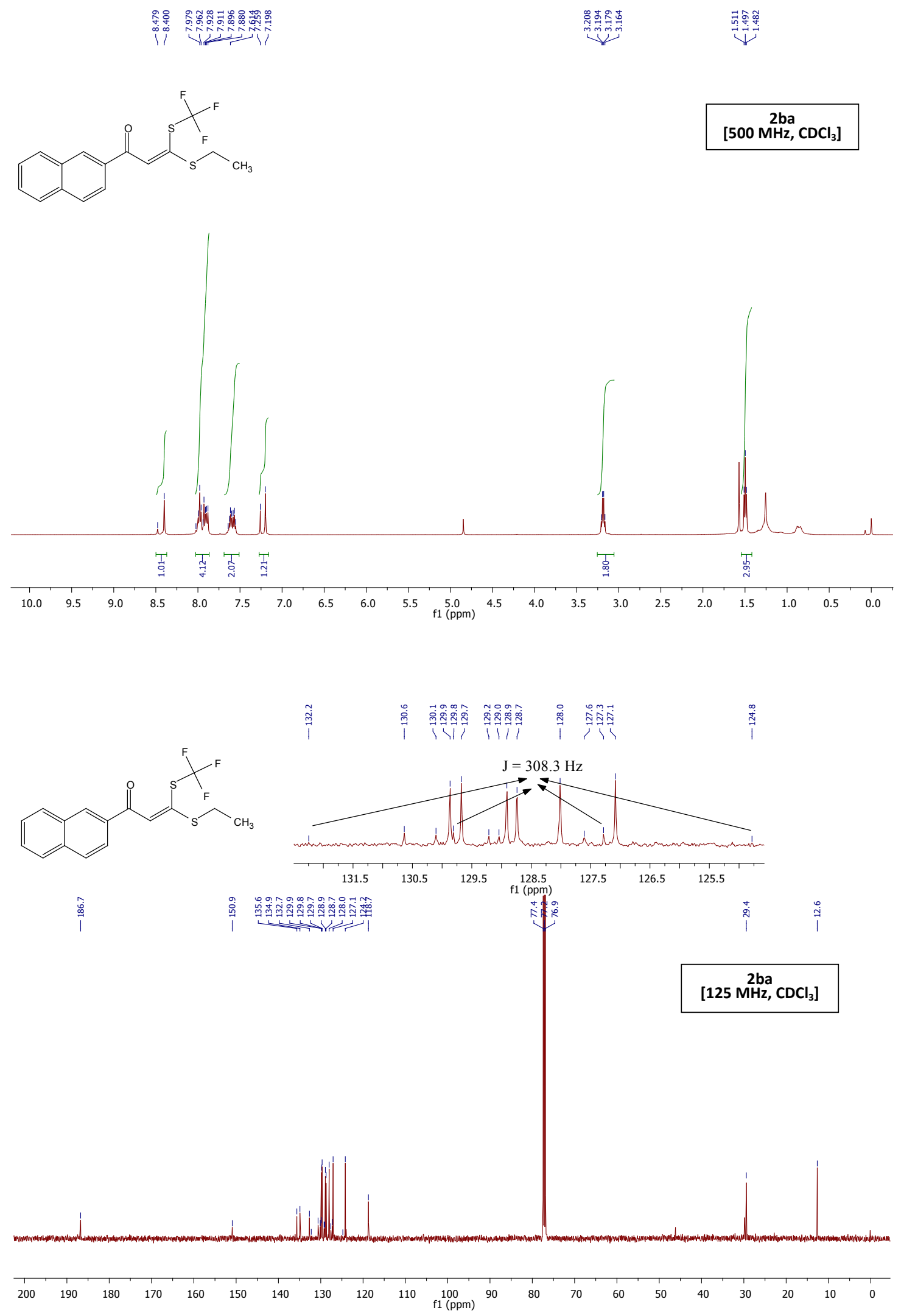





$\underline{{ }^{1} \mathrm{H} \text { NMR, }{ }^{13} \mathrm{C}\left\{{ }^{1} \mathrm{H}\right\} \text { NMR and }{ }^{19} \mathrm{~F} \text { NMR Spectra of Compound } \mathbf{2 b b}}$
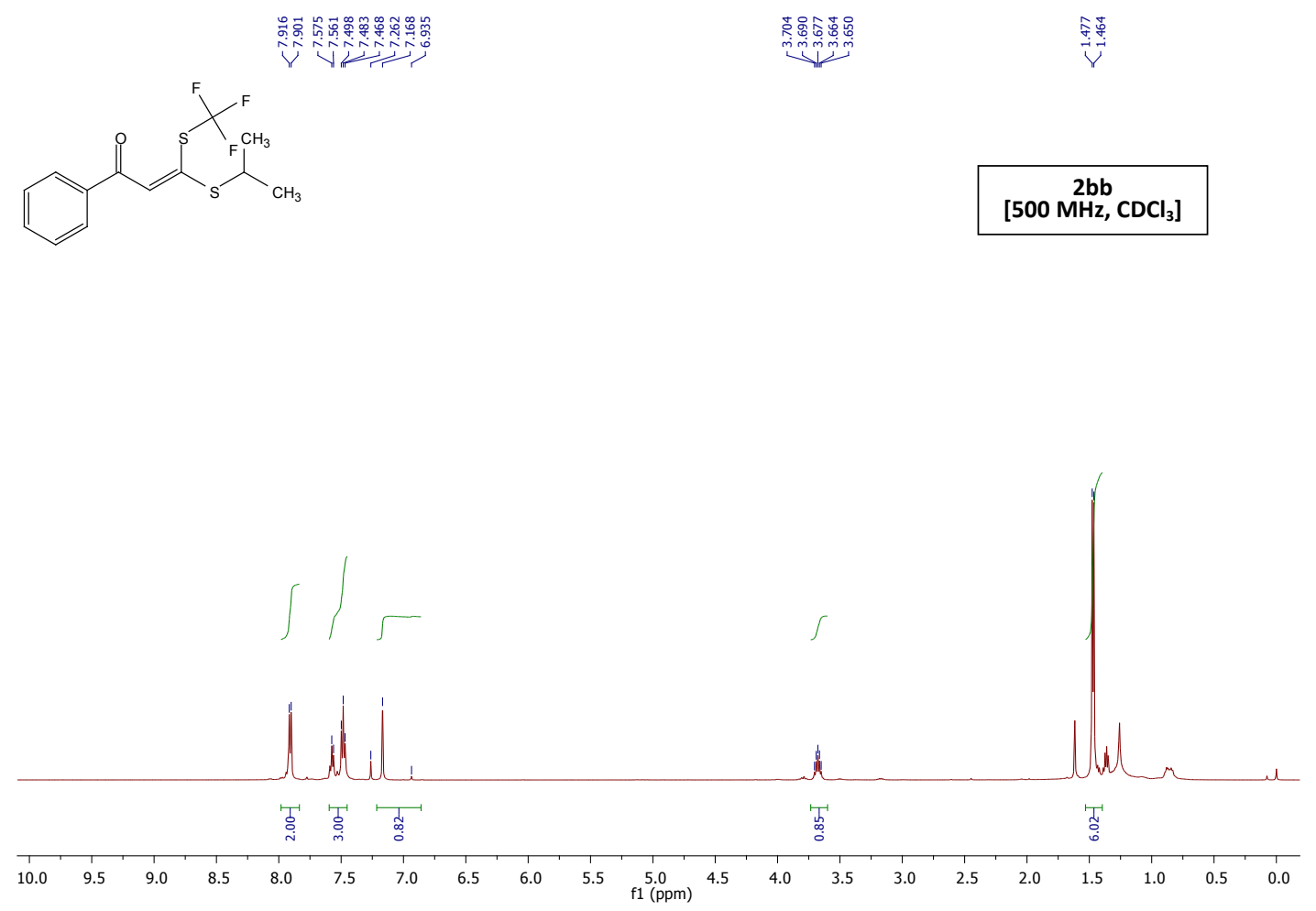


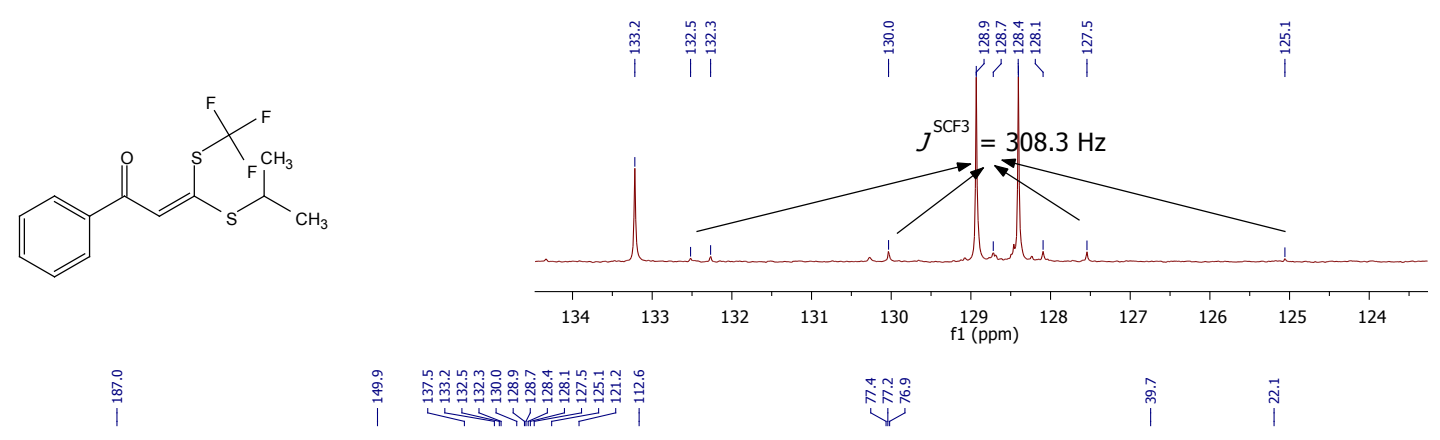

$2 b b$

[125 MHz, $\mathrm{CDCl}_{3}$ ]
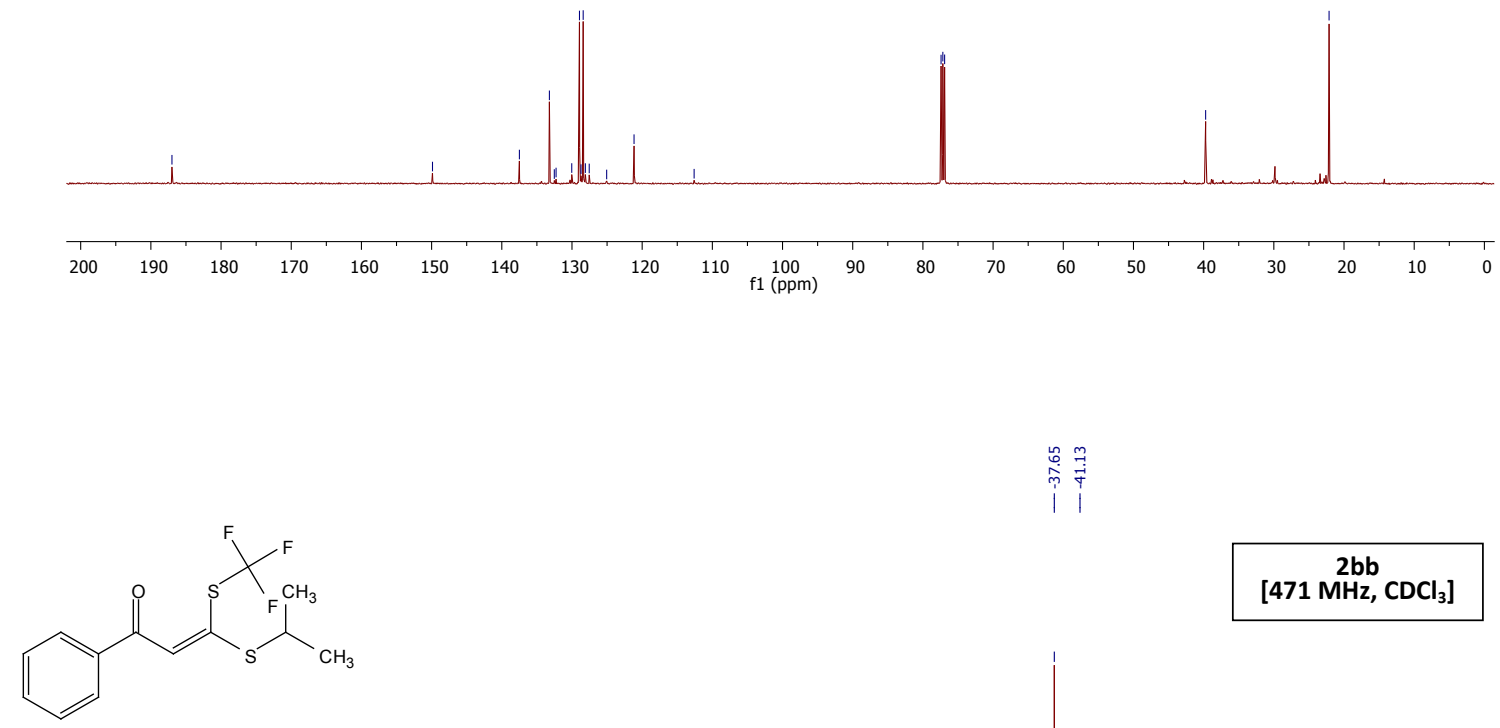

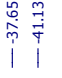

$\left[471 \mathrm{MHz}, \mathrm{CDCl}_{3}\right]$

$\begin{array}{llllllllllllllllllllll}1 & 100 & 90 & 80 & 70 & 60 & 50 & 40 & 30 & 20 & 10 & \underset{f}{1}(\mathrm{ppm}) & -10 & -20 & -30 & -40 & -50 & -60 & -70 & -80 & -90 & -100\end{array}$


$\underline{{ }^{1} \mathrm{H} N M R,{ }^{13} \mathrm{C}\left\{{ }^{1} \mathrm{H}\right\} \text { NMR and }{ }^{19} \mathrm{~F} \text { NMR Spectra of Compound } \mathbf{2 b c}}$
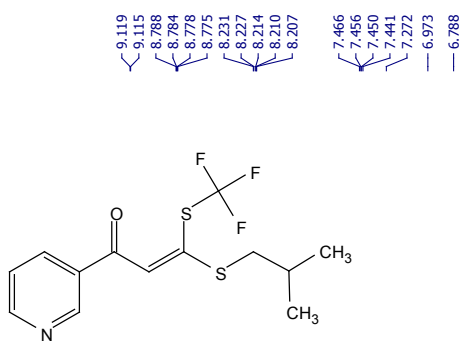

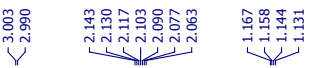
$\left[500 \mathrm{MHz}^{2 \mathrm{bc}} \mathrm{CDCl}_{3}\right]$
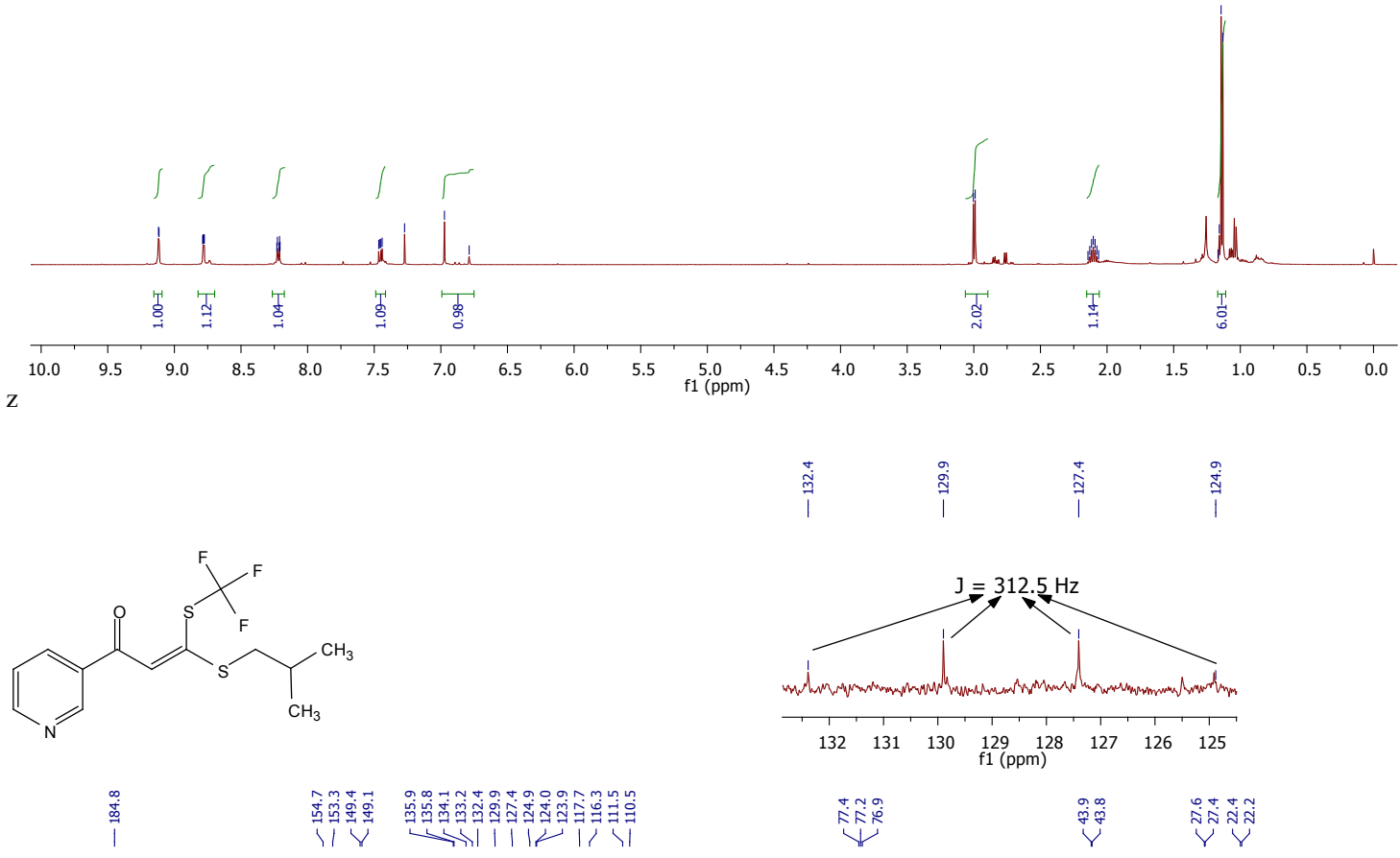

$2 b c$ [125 $\mathrm{MHz}^{2 \mathrm{bc}} \mathrm{CDCl}_{3}$ ]

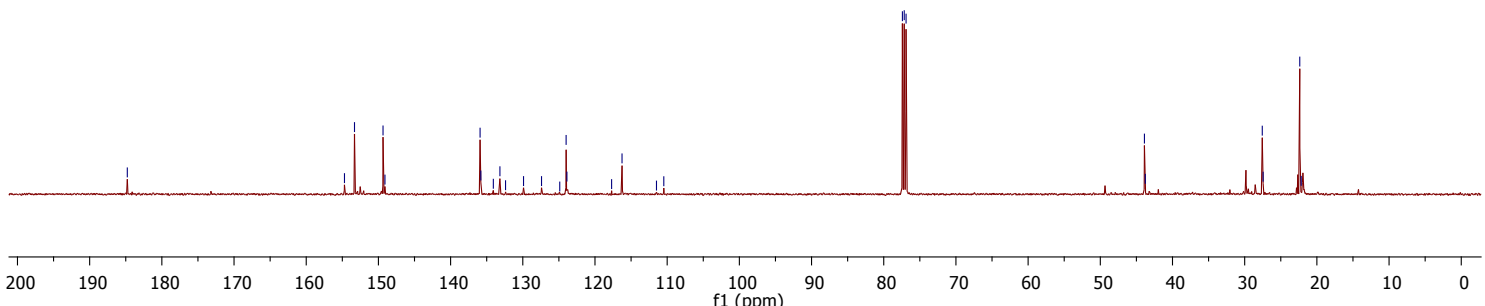



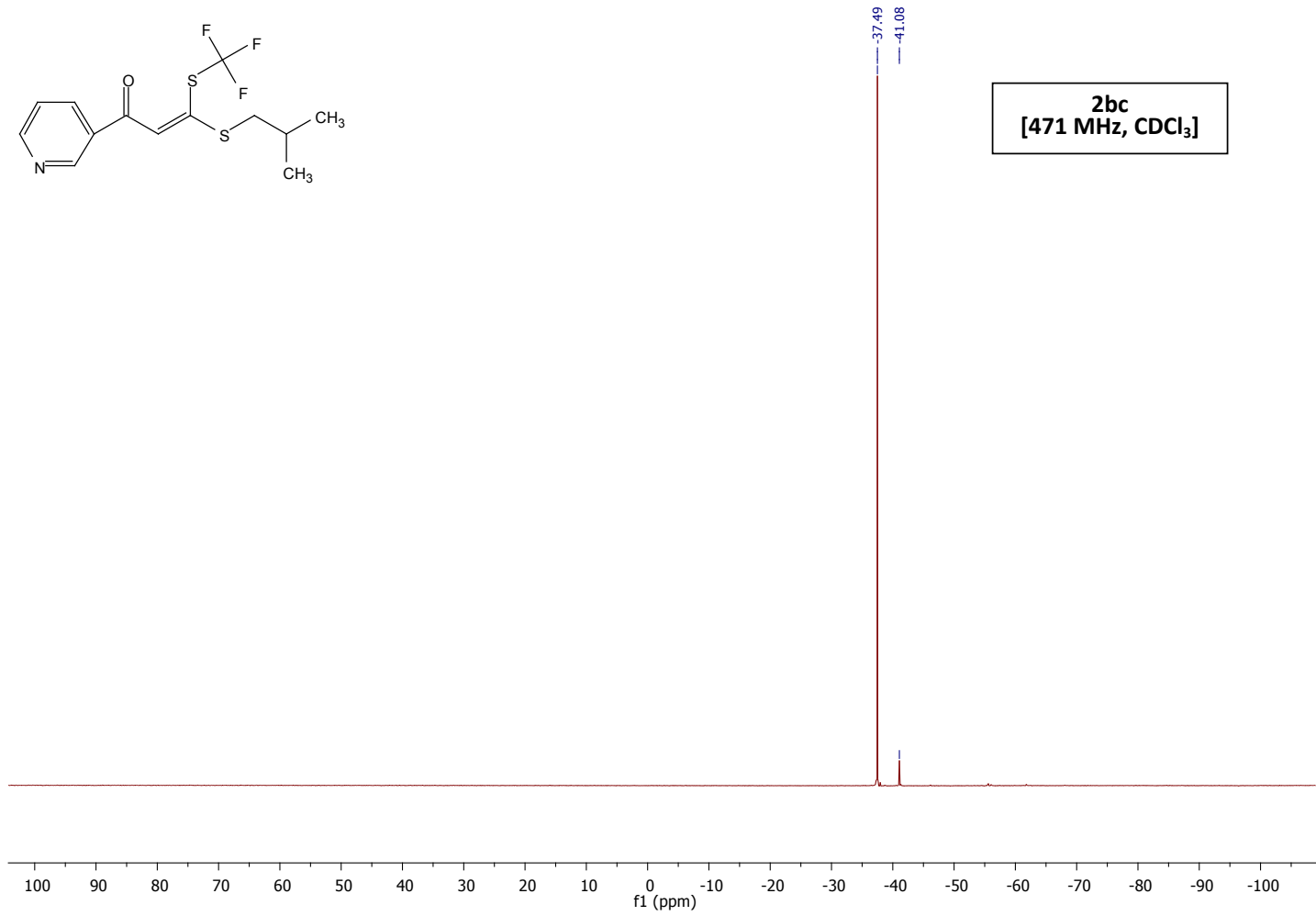

\section{$\underline{{ }^{1} \mathrm{H} \text { NMR, }{ }^{13} \mathrm{C}\left\{{ }^{1} \mathrm{H}\right\} \text { NMR and }{ }^{19} \mathrm{~F} \text { NMR Spectra of Compound } 2 \mathrm{bd}}$}
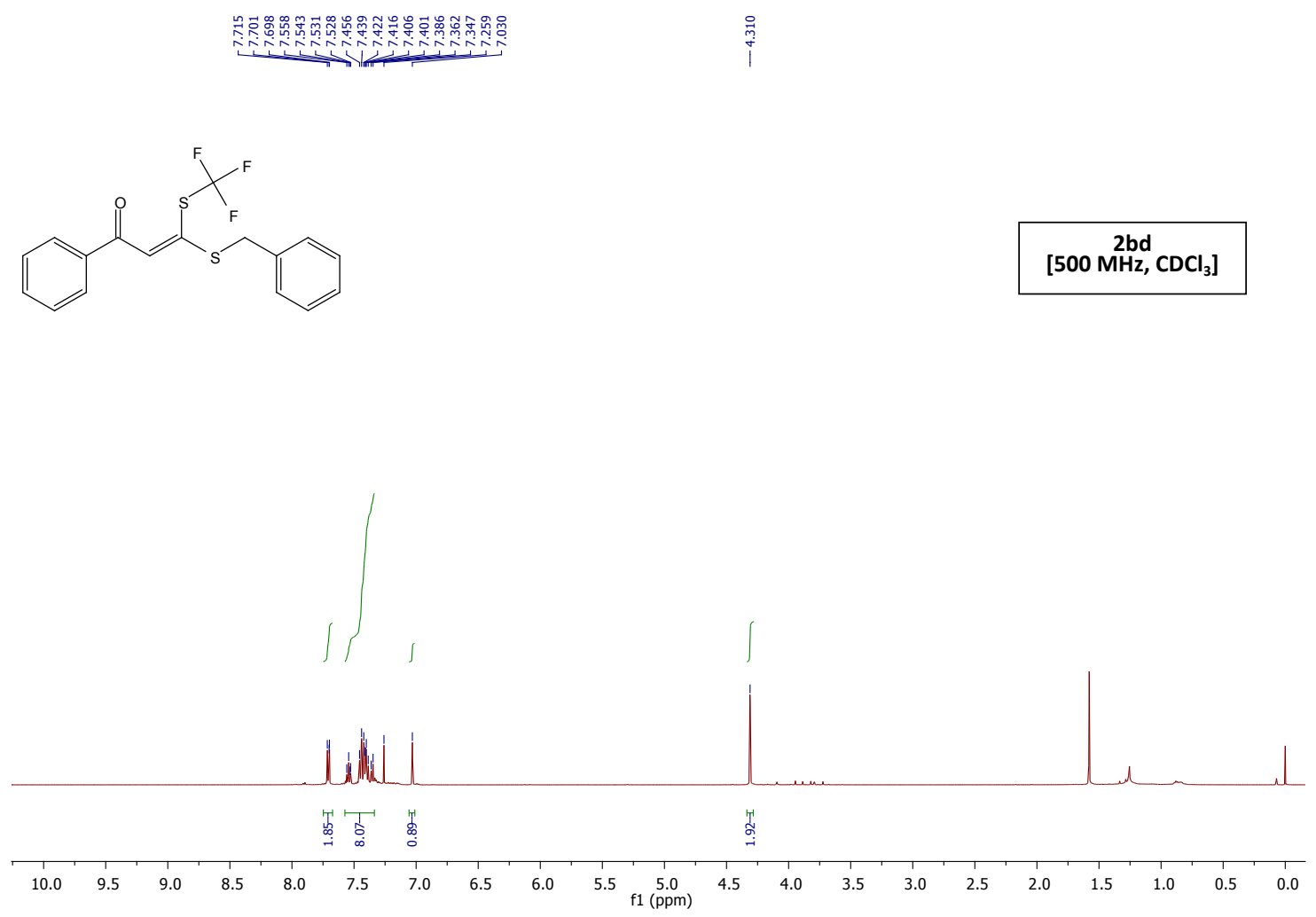

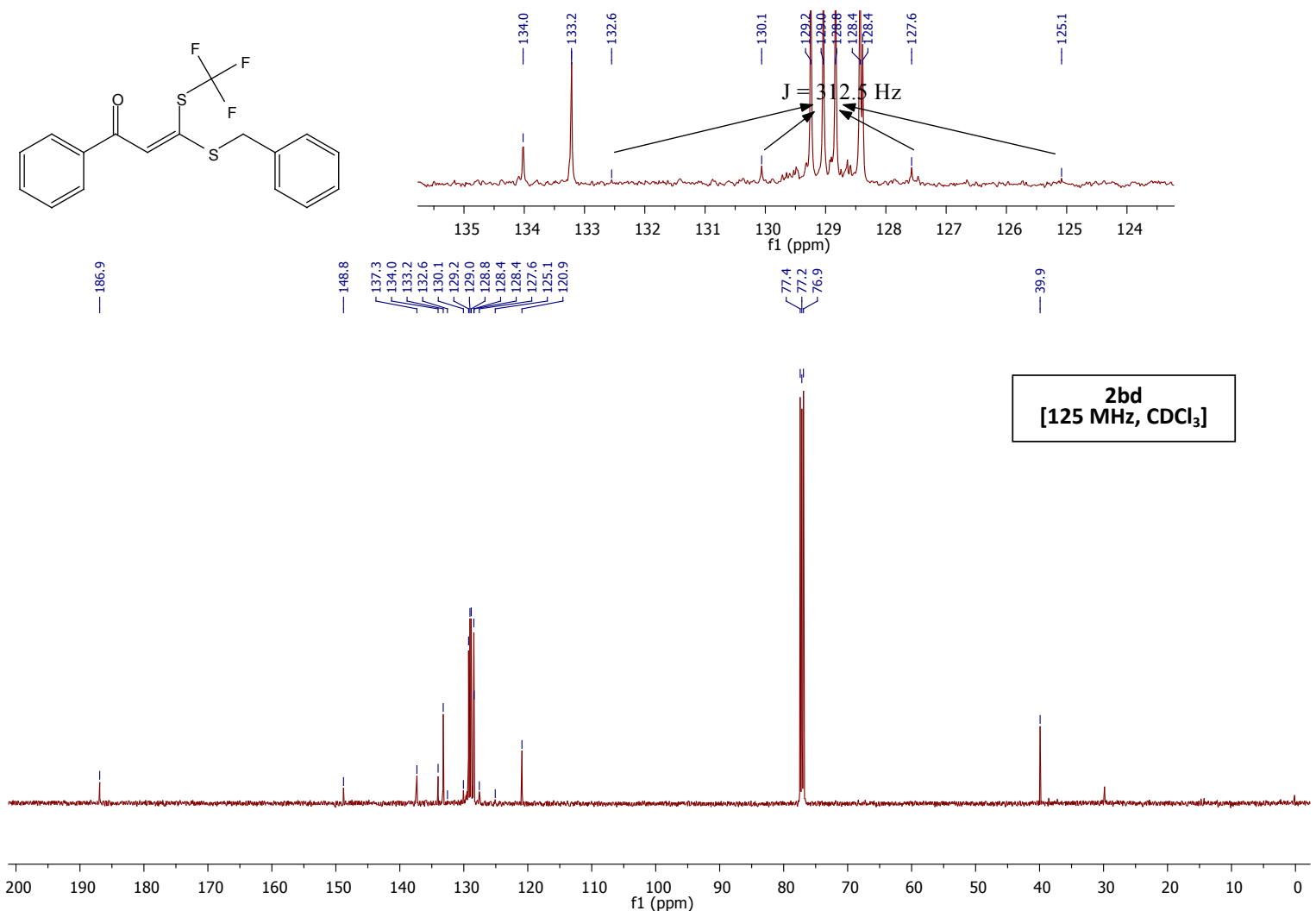

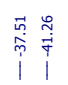

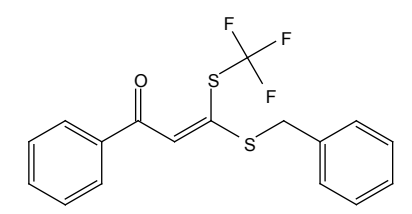

2bd
$\left[471 \mathrm{MHz}^{2}, \mathrm{CDCl}_{3}\right.$ ]





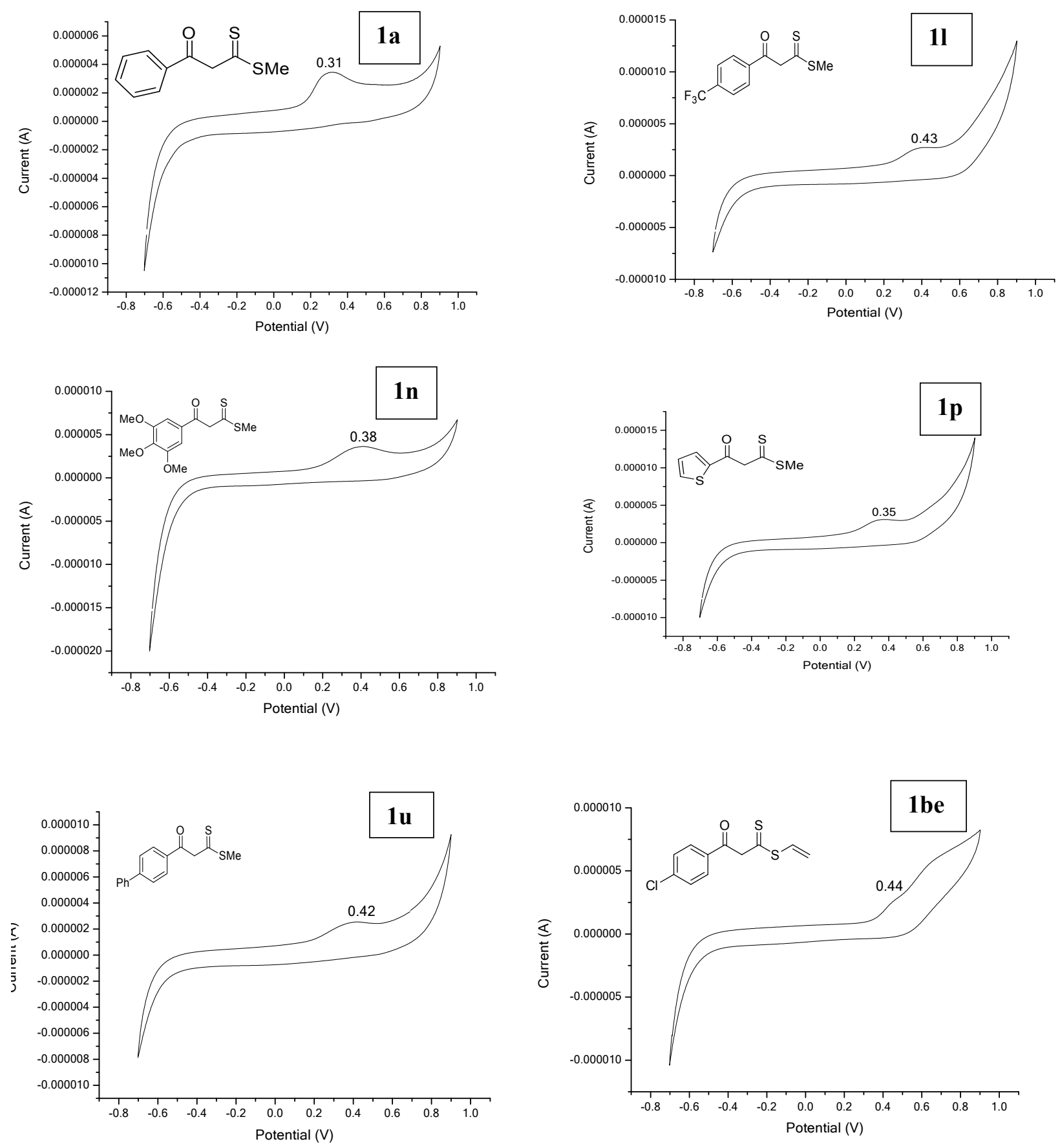

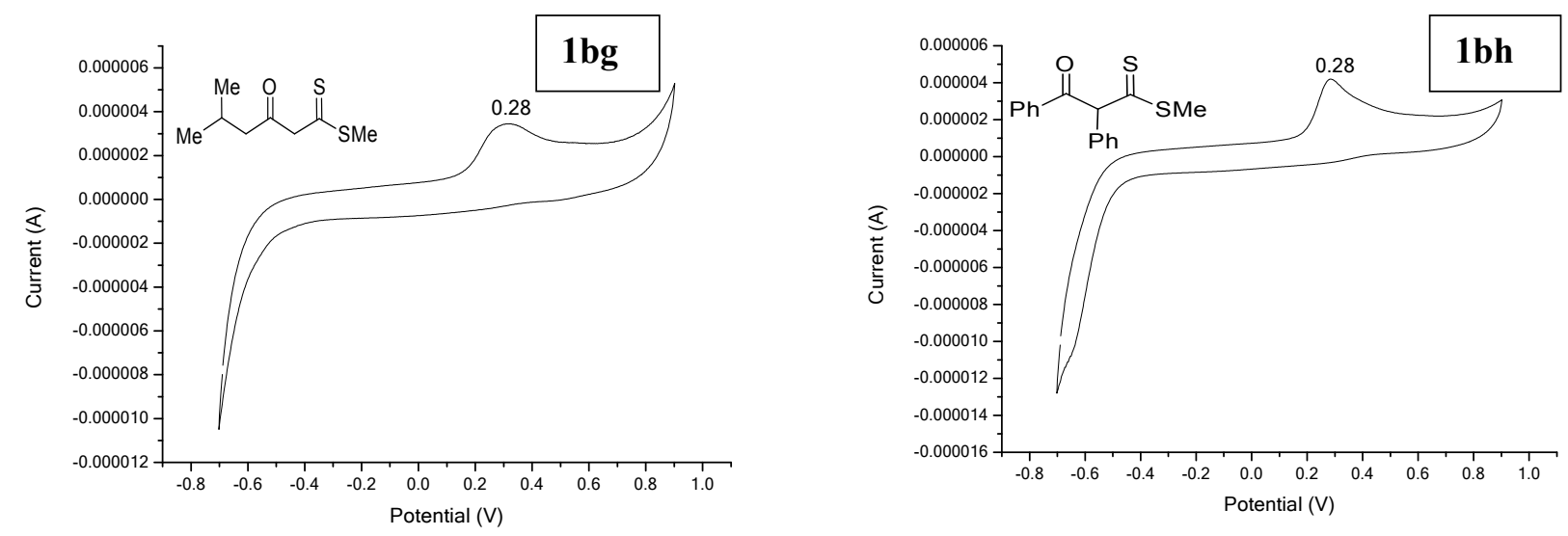

Figure S9: Cyclic Voltammogram of different $\beta$-ketodithioesters (1) were recorded in $0.1 \mathrm{M}$ TBAP/DMF electrolyte at $50 \mathrm{mV} / \mathrm{s}$ scan rate. Working electrode: glassy carbon electrode tip (3mm diameter); Counter electrode: Platinum wire; Reference electrode: $\mathrm{Ag} / \mathrm{AgCl}$.

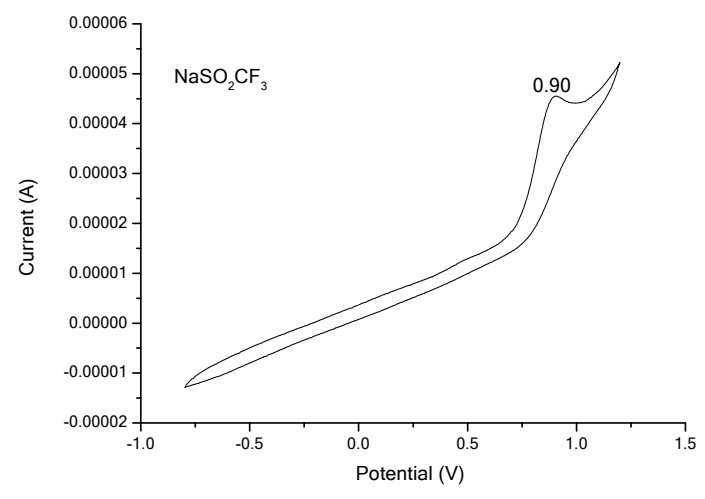

Figure S10: Cyclic Voltammogram of Sodium triflinate recorded in $0.1 \mathrm{M}$ TBAP/DMF electrolyte at $50 \mathrm{mV} / \mathrm{s}$ scan rate. Working electrode: glassy carbon electrode tip (3mm diameter); Counter electrode: Platinum wire; Reference electrode: $\mathrm{Ag} / \mathrm{AgCl}$.

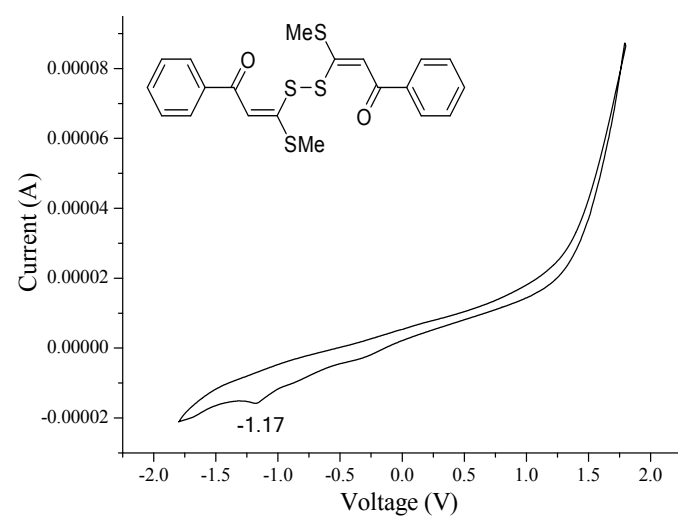

Figure S11: Cyclic Voltammogram of Dimer B of $\beta$-kDTE 1a recorded in $0.1 \mathrm{M}$ TBAP/DMF electrolyte at $100 \mathrm{mV} / \mathrm{s}$ scan rate. Working electrode: glassy carbon electrode tip (3mm diameter); Counter electrode: Platinum wire; Reference electrode: $\mathrm{Ag} / \mathrm{AgCl}$. 


\section{ORTEP Diagram of Compound 2s (CCDC 1949822)}
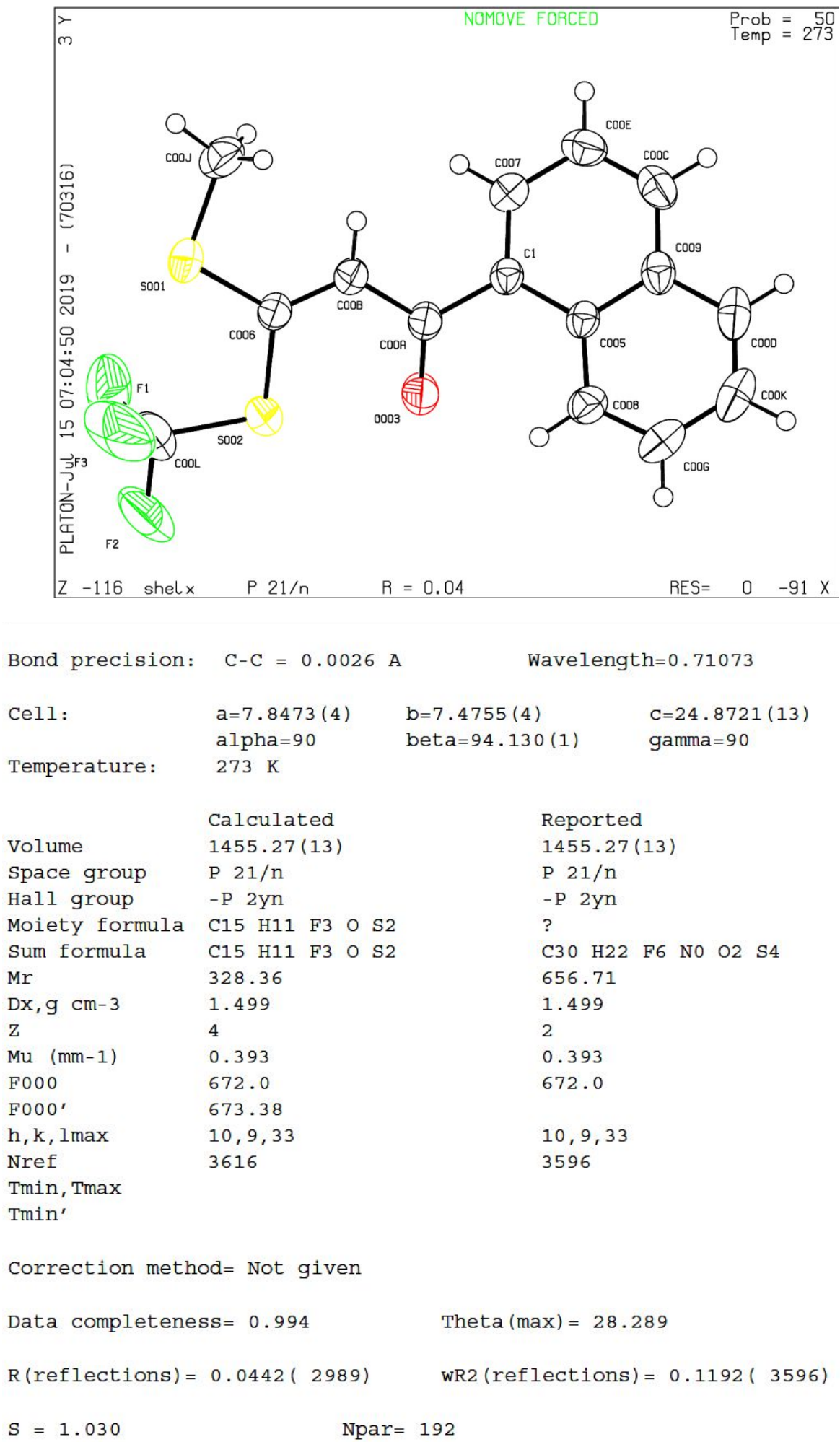


\section{Crystallization Details}

For the crystallization of compound $\mathbf{2 s}$, vacuum dried pure sample of $2 \mathbf{s}$ was taken in a vial and dissolved in $2 \mathrm{~mL}$ of acetonitrile. The vial was kept around $18-20{ }^{\circ} \mathrm{C}$ temperature for slow evaporation. After 5 days, we could find pale yellow needle shape crystal, which was picked up from the vial and performed single crystal XRD study. Analysis of the data exhibited that the particular crystal belongs to Z-isomer of $\mathbf{2 s .}$

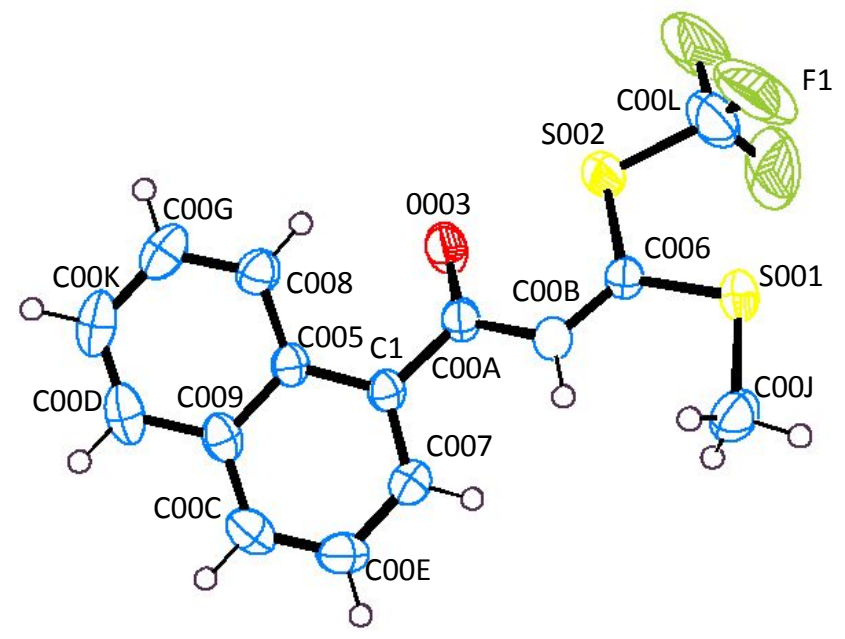

Figure S12. Thermal Ellipsoid Plot of Compound 2s (CCDC 1949822) 